International Journal of Pure and Applied Mathematics

Volume 83 No. 1 2013, 137-192

ISSN: 1311-8080 (printed version); ISSN: 1314-3395 (on-line version)

url: http://www.ijpam.eu

doi: http://dx.doi.org/10.12732/ijpam.v83i1.12

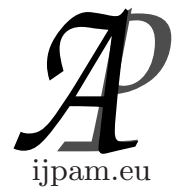

\title{
AXIOMATIC DIFFERENTIAL GEOMETRY II-3 \\ - ITS DEVELOPMENTS - \\ CHAPTER 3: THE GENERAL JACOBI IDENTITY
}

\author{
Hirokazu Nishimura \\ Institute of Mathematics \\ University of Tsukuba \\ Tsukuba, Ibaraki, 305-8571, JAPAN
}

\begin{abstract}
As the fourth paper of our series of papers concerned with axiomatic differential geometry, this paper is devoted to the general Jacobi identity supporting the Jacobi identity of vector fields. The general Jacobi identity can be regarded as one of the few fundamental results belonging properly to smootheology.
\end{abstract}

AMS Subject Classification: 58A05

Key Words: axiomatic differential geometry, general Jacobi identity, strong difference, Jacobi identity, vector fields

\section{Introduction}

It is well known in traditional differential geometry that the totality of vector fields on a smooth manifold forms a Lie algebra. The proof of this fact is tremendously easy, because we can identity vector fields with derivations within the particular category that orthodox differential geometers have indulged in. An axiomatic treatment of differential geometry emancipates differential geometers from this comfortable adherence to their favorite category of smooth manifolds and forces them to confront the infinitesimal structure per se barehanded.

The Jacobi identity occupies the central position in the structure of a Lie algebra, and we stumbled upon the general Jacobi identity supporting the Jacobi identity of vector fields from behind the very vale of infinitesimal structures

Received: December 6, 2012

(C) 2013 Academic Publications, Ltd. url: www.acadpubl.eu 
within the framework of synthetic differential geometry in the previous century, for which the reader is referred to [2], [3] and [4]. This paper is devoted to the general Jacobi identity within our axiomatics of differential geometry, which will play a predominant role in a subsequent paper dealing with the FrölicherNijenhuis calculus.

Our axiomatic differential geometry is an attempt to grasp the infinitesimal structure without fringes or frills. It seems that the term "smootheology" or "diffeology" is gaining momentum for the study of such an infinitesimal structure. We think that the general Jacobi identity is one of the few fundamental results indigenous to smootheology. This infinitesimal structure lies at the very core of not only differential geometry but also many pure or applied branches of mathematics. We assume that the reader is familiar with our axiomatic framework of differential geometry presented in [7] and [9]. Thus we are working within a DG-category

$$
(\mathcal{K}, \mathbb{R}, \mathbf{T}, \alpha)
$$

in the sense of [9]. We always assume that $M$ is a microlinear and Weilexponentiable object in the category $\mathcal{K}$.

The general Jacobi identity will be dealt with in Section 4, which will be preceded by the more elementary treatment of the primordial general Jacobi identity in Section 3. The final section is devoted to the derivation of the Jacobi identity of vector fields from the general Jacobi identity, in which the reader is assumed to be familiar with [8].

\section{Simplicial Sets}

We need to fix notation and terminology for simplicial objects, which form an important subclass of infinitesimal objects. Simplicial objects are infinitesimal objects of the form

$$
\begin{aligned}
& D^{n}\{\mathbf{p}\} \\
& =\left\{\left(d_{1}, \ldots, d_{n}\right) \in D^{n} \mid d_{i_{1}} \ldots d_{i_{k}}=0\left(\forall\left(i_{1}, \ldots, i_{k}\right) \in \mathbf{p}\right)\right\}
\end{aligned}
$$

where $\mathbf{p}$ is a finite set of finite sequences $\left(i_{1}, \ldots, i_{k}\right)$ of natural numbers between 1 and $n$, including the endpoints, with $i_{1}<\ldots<i_{k}$. If $\mathrm{p}$ is empty, $D^{n}\{\mathrm{p}\}$ is $D^{n}$ itself. If $\mathrm{p}$ consists of all the binary sequences, then $D^{n}\{\mathrm{p}\}$ represents $D(n)$ in the standard terminology of SDG. Given two simplicial objects $D^{m}\{\mathrm{p}\}$ and $D^{n}\{\mathbf{q}\}$, we define a simplicial object $D^{m}\{\mathbf{p}\} \oplus D^{n}\{\mathbf{q}\}$ to be

$$
D^{m+n}\{\mathbf{p} \oplus \mathbf{q}\}
$$


where

$$
\begin{gathered}
\mathbf{p} \oplus \mathbf{q} \\
=\mathbf{p} \cup\left\{\left(j_{1}+m, \ldots, j_{k}+m\right) \mid\left(j_{1}, \ldots, j_{k}\right) \in \mathbf{q}\right\} \\
\cup\{(i, j+m) \mid 1 \leq i \leq m, 1 \leq j \leq n\}
\end{gathered}
$$

Since the operation $\oplus$ is associative, we can combine any finite number of simplicial objects by $\oplus$ without bothering about how to insert parentheses. Given morphisms of simplicial objects $\Phi_{i}: D^{m_{i}}\left\{\mathbf{p}_{i}\right\} \rightarrow D^{m}\{\mathbf{p}\}(1 \leq i \leq n)$, there exists a unique morphism of simplicial objects $\Phi: D^{m_{1}}\left\{\mathbf{p}_{1}\right\} \oplus \ldots \oplus D^{m_{n}}\left\{\mathbf{p}_{n}\right\} \rightarrow$ $D^{m}\{\mathbf{p}\}$ whose restriction to $D^{m_{i}}\left\{\mathbf{p}_{i}\right\}$ coincides with $\Phi_{i}$ for each $i$. We denote this $\Phi$ by $\Phi_{1} \oplus \ldots \oplus \Phi_{n}$. We write $D(n)$ for $\left\{(d, \ldots, d) \in D^{n} \mid d_{i} d_{j}=0\right.$ for any $i \neq j\}$.

\section{The Preliminary Identity}

The principal objective in this paper is to give the general Jacobi identity and its proof. Our harder treatment of the general Jacobi identity in the coming section is preceded by a simpler treatment of the primordial general Jacobi identity in this section, because the latter is easy to grasp intuitively so that it prepares the reader for the coming general Jacobi identity.

Proposition 1. The diagram

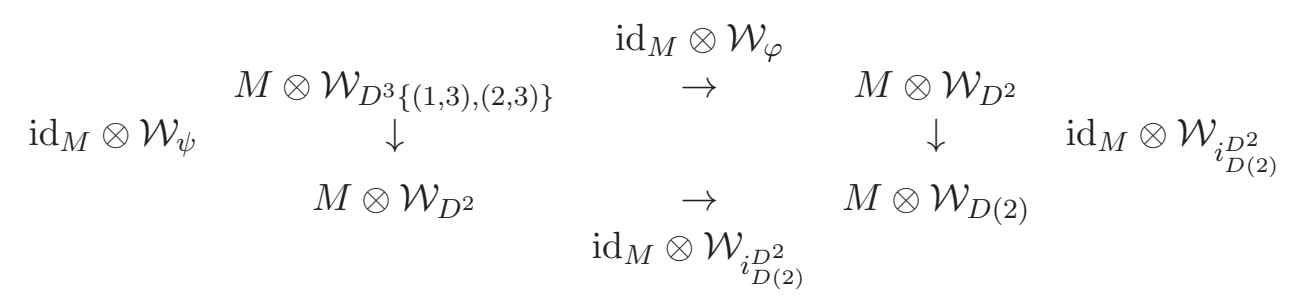

is a pullback diagram, where the assumptive mapping $\varphi: D^{2} \rightarrow D^{3}\{(1,3),(2,3)\}$ is

$$
\left(d_{1}, d_{2}\right) \in D^{2} \mapsto\left(d_{1}, d_{2}, 0\right) \in D^{3}\{(1,3),(2,3)\}
$$

while the assumptive mapping $\psi: D^{2} \rightarrow D^{3}\{(1,3),(2,3)\}$ is

$$
\left(d_{1}, d_{2}\right) \in D^{2} \mapsto\left(d_{1}, d_{2}, d_{1} d_{2}\right) \in D^{3}\{(1,3),(2,3)\}
$$


Proof. This follows from the microlinearity of $M$ and the pullback diagram of Weil algebras

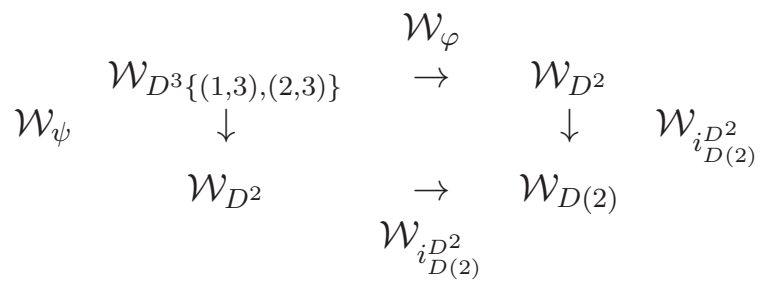

Corollary 2. We have

$$
M \otimes \mathcal{W}_{D^{3}\{(1,3),(2,3)\}}=\left(M \otimes \mathcal{W}_{D^{2}}\right) \times_{M \otimes \mathcal{W}_{D(2)}}\left(M \otimes \mathcal{W}_{D^{2}}\right)
$$

Notation 3. We will write

$$
\zeta^{-}:\left(M \otimes \mathcal{W}_{D^{2}}\right) \times_{M \otimes \mathcal{W}_{D(2)}}\left(M \otimes \mathcal{W}_{D^{2}}\right) \rightarrow M \otimes \mathcal{W}_{D}
$$

for the morphism

$$
\begin{aligned}
& \left(\operatorname{id}_{M} \otimes \mathcal{W}_{d \in D \mapsto(0,0, d) \in D^{3}\{(1,3),(2,3)\}}, \mathcal{W}_{i_{D(2)}^{D^{2}}} \circ \mathcal{W}_{\varphi}\right) \\
& : \mathcal{W}_{D^{2}} \times \mathcal{W}_{D(2)} \mathcal{W}_{D^{2}}=\mathcal{W}_{D^{2}} \times_{\mathcal{W}_{D(2)}} \mathcal{W}_{D^{2}} \rightarrow M \otimes \mathcal{W}_{D}
\end{aligned}
$$

The following is the prototype for the general Jacobi identity.

Theorem 4. (The Primordial General Jacobi Identity) The three morphisms

$$
\begin{aligned}
& \zeta^{*_{2}-*_{1}}:\left(M \otimes \mathcal{W}_{D^{2}}\right) \times_{M} \otimes \mathcal{W}_{D(2)}\left(M \otimes \mathcal{W}_{D^{2}}\right) \times_{M \otimes \mathcal{W}_{D(2)}}\left(M \otimes \mathcal{W}_{D^{2}}\right) \\
& \rightarrow \underset{1}{\left(M \otimes \mathcal{W}_{D^{2}}\right) \times \times_{M \otimes \mathcal{W}_{D(2)}}}\left(M \otimes \mathcal{W}_{D^{2}}\right) \underline{\zeta^{-}}\left(M \otimes \mathcal{W}_{D}\right) \times\left(M \otimes \mathcal{W}_{D(2)}\right) \\
& \zeta^{*_{3}-*_{2}}:\left(M \otimes \underset{1}{\otimes} \mathcal{W}_{D^{2}}\right) \times_{M \otimes} \mathcal{W}_{D(2)}\left(M \otimes \mathcal{W}_{D^{2}}\right) \times_{M \otimes \mathcal{W}_{D(2)}}\left(M \otimes \mathcal{W}_{D^{2}}\right) \\
& \rightarrow \underset{2}{\left(M \otimes \mathcal{W}_{D^{2}}\right) \times \times_{M \otimes \mathcal{W}_{D(2)}}}\left(M \otimes \mathcal{W}_{D^{2}}\right) \underset{\zeta}{\zeta}\left(M \otimes \mathcal{W}_{D}\right) \times\left(M \otimes \mathcal{W}_{D(2)}\right) \\
& \zeta^{*_{1}-*_{3}}:\left(M \otimes \mathcal{W}_{D^{2}}\right) \times_{M \otimes} \mathcal{W}_{D(2)}\left(M \otimes \mathcal{W}_{D^{2}}\right) \times_{M \otimes \mathcal{W}_{D(2)}}\left(M \otimes \mathcal{W}_{D^{2}}\right)
\end{aligned}
$$




$$
\rightarrow \underset{3}{\left(M \mathcal{W}_{D^{2}}\right) \times} \times_{M \otimes \mathcal{W}_{D(2)}}\left(M \otimes \mathcal{W}_{D^{2}}\right) \underset{\zeta^{-}}{\longrightarrow}\left(M \otimes \mathcal{W}_{D}\right) \times\left(M \otimes \mathcal{W}_{D(2)}\right)
$$

sum up only to vanish, where the numbers under $\left(M \otimes \mathcal{W}_{D^{2}}\right)$ are given simply so as for the reader to relate each occurrence of $\left(M \otimes \mathcal{W}_{D^{2}}\right)$ to another, and the unlabeled arrows are the canonical projections.

The proof of Theorem 4 is based completely upon the following theorem.

Theorem 5. The diagram

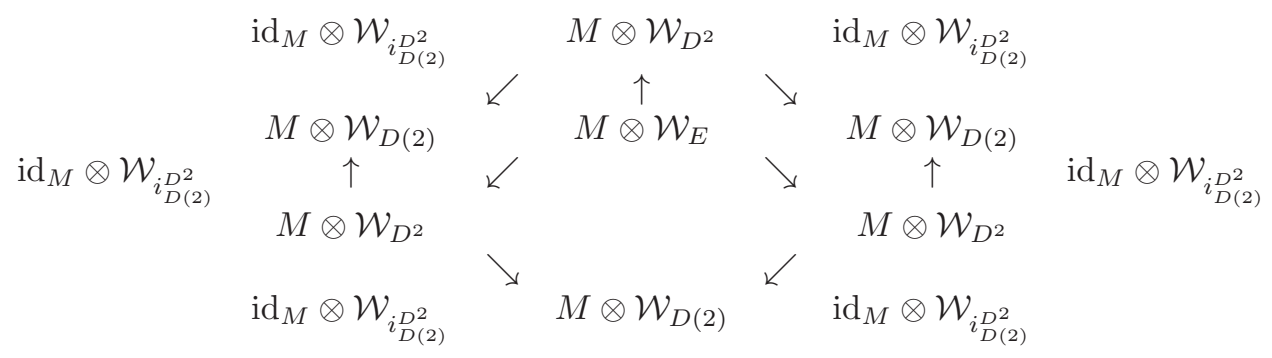

is a limit diagram, where the assumptive object $E$ is

$$
D^{4}\{(1,3),(2,3),(1,4),(2,4),(3,4)\}
$$

and the assumptive mapping $i_{D(2)}^{D^{2}}: D(2) \rightarrow D^{2}$ is $\left(d_{1}, d_{2}\right) \in D(2) \mapsto\left(d_{1}, d_{2}\right) \in$ $D^{2}$, while the three unnamed arrows $M \otimes \mathcal{W}_{E} \rightarrow M \otimes \mathcal{W}_{D^{2}}$ are $\operatorname{id}_{M} \otimes \mathcal{W}_{l_{i}}$ $(i=1,2,3)$ counterclockwise from the top with the assumptive mappings $l_{i}$ : $D^{2} \rightarrow E(i=1,2,3)$ being

$$
\begin{aligned}
& l_{1}:\left(d_{1}, d_{2}\right) \in D^{2} \mapsto\left(d_{1}, d_{2}, 0,0\right) \in E \\
& l_{2}:\left(d_{1}, d_{2}\right) \in D^{2} \mapsto\left(d_{1}, d_{2}, d_{1} d_{2}, 0\right) \in E \\
& l_{3}:\left(d_{1}, d_{2}\right) \in D^{2} \mapsto\left(d_{1}, d_{2}, 0, d_{1} d_{2}\right) \in E
\end{aligned}
$$

Corollary 6. We have

$$
\begin{aligned}
& \left(M \otimes \mathcal{W}_{D^{2}}\right) \times_{M \otimes \mathcal{W}_{D(2)}}\left(M \otimes \mathcal{W}_{D^{2}}\right) \times_{M \otimes \mathcal{W}_{D(2)}}\left(M \otimes \mathcal{W}_{D^{2}}\right) \\
& =M \otimes \mathcal{W}_{E}
\end{aligned}
$$

This theorem follows directly from the following lemma. 
Lemma 7. The following diagram is a limit diagram of Weil algebras:

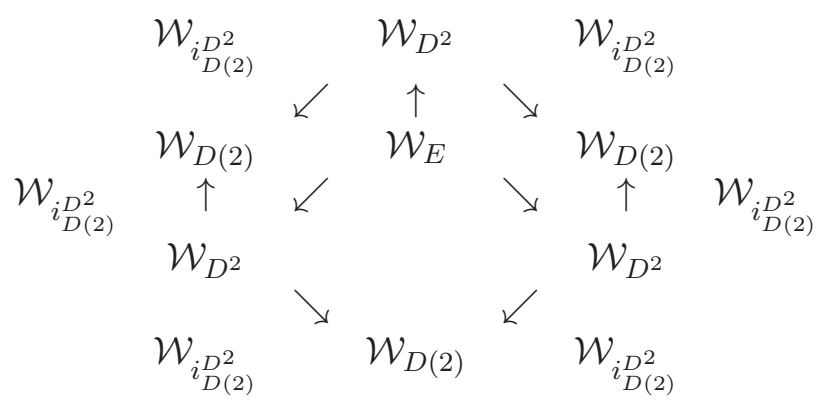

Proof. Let $\gamma_{1}, \gamma_{2}, \gamma_{3} \in \mathcal{W}_{D^{2}}$ and $\gamma \in \mathcal{W}_{E}$ so that they are the polynomials with coefficients in $k$ of the following forms:

$$
\begin{aligned}
\gamma_{1}\left(X_{1}, X_{2}\right) & =a+a_{1} X_{1}+a_{2} X_{2}+a_{12} X_{1} X_{2} \\
\gamma_{2}\left(X_{1}, X_{2}\right) & =b+b_{1} X_{1}+b_{2} X_{2}+b_{12} X_{1} X_{2} \\
\gamma_{3}\left(X_{1}, X_{2}\right) & =c+c_{1} X_{1}+c_{2} X_{2}+c_{12} X_{1} X_{2} \\
\gamma\left(X_{1}, X_{2}, X_{3}, X_{4}\right) & =e+e_{1} X_{1}+e_{2} X_{2}+e_{12} X_{1} X_{2}+e_{3} X_{3}+e_{4} X_{4}
\end{aligned}
$$

The condition that $\mathcal{W}_{i_{D(2)}^{D^{2}}}\left(\gamma_{1}\right)=\mathcal{W}_{i_{D(2)}^{D^{2}}}\left(\gamma_{2}\right)=\mathcal{W}_{i_{D(2)}^{D^{2}}}\left(\gamma_{3}\right)$ is equivalent to the following three conditions as a whole:

$$
\begin{gathered}
a=b=c \\
a_{1}=b_{1}=c_{1} \\
a_{2}=b_{2}=c_{2}
\end{gathered}
$$

Therefore, in order that $\mathcal{W}_{l_{1}}(\gamma)=\gamma_{1}, \mathcal{W}_{l_{2}}(\gamma)=\gamma_{2}$ and $\mathcal{W}_{l_{3}}(\gamma)=\gamma_{3}$ in this case, it is necessary and sufficient that the polynomial $\gamma$ should be of the following form:

$\gamma\left(X_{1}, X_{2}, X_{3}, X_{4}\right)=a+a_{1} X_{1}+a_{2} X_{2}+a_{12} X_{1} X_{2}+\left(b_{12}-a_{12}\right) X_{3}+\left(c_{12}-a_{12}\right) X_{4}$

This completes the proof.

Theorem 8. The diagram

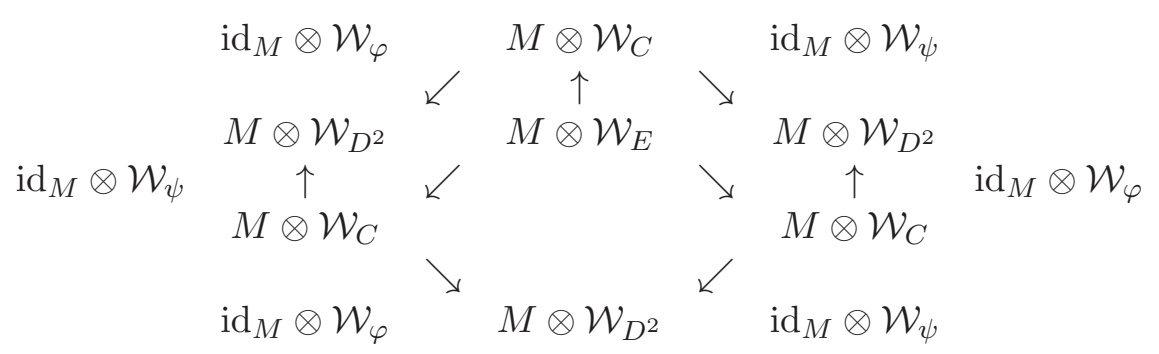


is a limit diagram, where $C$ stands for

$$
D^{3}\{(1,3),(2,3)\}
$$

and the three unnamed morphisms go contraclockwise from the top as follows:

$$
\begin{aligned}
& \operatorname{id}_{M} \otimes \mathcal{W}_{\left(d_{1}, d_{2}, d_{3}\right) \in D^{3}\{(1,3),(2,3)\} \mapsto\left(d_{1}, d_{2}, d_{3}, 0\right) \in E}: M \otimes \mathcal{W}_{E} \rightarrow M \otimes \mathcal{W}_{C} \\
& \operatorname{id}_{M} \otimes \mathcal{W}_{\left(d_{1}, d_{2}, d_{3}\right) \in D^{3}\{(1,3),(2,3)\} \mapsto\left(d_{1}, d_{2}, d_{1} d_{2}-d_{3}, d_{3}\right) \in E}: M \otimes \mathcal{W}_{E} \rightarrow M \otimes \mathcal{W}_{C} \\
& \operatorname{id}_{M} \otimes \mathcal{W}_{\left(d_{1}, d_{2}, d_{3}\right) \in D^{3}\{(1,3),(2,3)\} \mapsto\left(d_{1}, d_{2}, 0, d_{1} d_{2}-d_{3}\right) \in E}: M \otimes \mathcal{W}_{E} \rightarrow M \otimes \mathcal{W}_{C}
\end{aligned}
$$

This theorem follows directly from the following lemma.

Lemma 9. The diagram

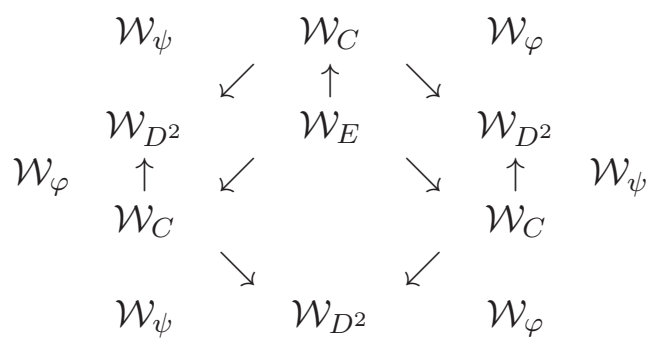

is a limit diagram, where the three unnamed morphisms go contraclockwise from the top as follows:

$$
\begin{aligned}
& \mathcal{W}_{\left(d_{1}, d_{2}, d_{3}\right) \in D^{3}\{(1,3),(2,3)\} \mapsto\left(d_{1}, d_{2}, d_{3}, 0\right) \in E}: \mathcal{W}_{E} \rightarrow \mathcal{W}_{C} \\
& \mathcal{W}_{\left(d_{1}, d_{2}, d_{3}\right) \in D^{3}\{(1,3),(2,3)\} \mapsto\left(d_{1}, d_{2}, d_{1} d_{2}-d_{3}, d_{3}\right) \in E}: \mathcal{W}_{E} \rightarrow \mathcal{W}_{C} \\
& \mathcal{W}_{\left(d_{1}, d_{2}, d_{3}\right) \in D^{3}\{(1,3),(2,3)\} \mapsto\left(d_{1}, d_{2}, 0, d_{1} d_{2}-d_{3}\right) \in E}: \mathcal{W}_{E} \rightarrow \mathcal{W}_{C}
\end{aligned}
$$

Proof. By the same token as in Lemma 7.

Proof. (of the primordial Jacobi identity). The morphism

$\zeta^{*_{2}-*_{1}}: \underset{1}{\left(M \otimes \mathcal{W}_{D^{2}}\right) \times_{M \otimes} \mathcal{W}_{D(2)}}\left(M \otimes \mathcal{W}_{D^{2}}\right) \times_{M \otimes} \mathcal{W}_{D(2)}\left(M \otimes \mathcal{W}_{D^{2}}\right) \rightarrow M \otimes \mathcal{W}_{D}$ is the composition of

$$
\begin{aligned}
& \operatorname{id}_{M} \otimes \mathcal{W}_{\left(d_{1}, d_{2}, d_{3}\right) \in D^{3}\{(1,3),(2,3)\} \mapsto\left(d_{1}, d_{2}, d_{3}, 0\right) \in E} \\
& :\left(M \otimes \mathcal{W}_{D^{2}}\right) \times_{M} \otimes \mathcal{W}_{D(2)}\left(M \otimes \mathcal{W}_{D^{2}}\right) \times_{M} \otimes \mathcal{W}_{D(2)} \\
& \quad\left(M \otimes \mathcal{W}_{D^{2}}\right) \\
& \quad=M \otimes \mathcal{W}_{E} \rightarrow M \otimes \mathcal{W}_{C}
\end{aligned}
$$




$$
=\left(M \otimes \mathcal{W}_{D^{2}}\right) \times_{M} \otimes \mathcal{W}_{D(2)}\left(M \otimes \mathcal{W}_{D^{2}}\right)
$$

and

$$
\dot{\zeta}^{-}: \underset{1}{\left(M \otimes \mathcal{W}_{D^{2}}\right) \times_{M} \otimes \mathcal{W}_{D(2)}} \underset{2}{\left(M \otimes \mathcal{W}_{D^{2}}\right)} \rightarrow M \otimes \mathcal{W}_{D}
$$

in succession, which is in turn equivalent to

$$
\operatorname{id}_{M} \otimes \mathcal{W}_{d \in D \mapsto(0,0, d, 0) \in E}
$$

The morphism

$\zeta^{*_{3}-*_{2}}:\left(M \otimes \mathcal{W}_{D^{2}}\right) \times_{M \otimes} \mathcal{W}_{D(2)}\left(M \otimes \mathcal{W}_{D^{2}}\right) \times_{M \otimes \mathcal{W}_{D(2)}}\left(M \otimes \mathcal{W}_{D^{2}}\right) \rightarrow M \otimes \mathcal{W}_{D}$ is the composition of

$$
\begin{aligned}
& \operatorname{id}_{M} \otimes \mathcal{W}_{\left(d_{1}, d_{2}, d_{3}\right) \in D^{3}\{(1,3),(2,3)\} \mapsto\left(d_{1}, d_{2}, d_{1} d_{2}-d_{3}, d_{3}\right) \in E} \\
& :\left(M \otimes \mathcal{W}_{D^{2}}\right) \times_{M \otimes \mathcal{W}_{D(2)}}\left(M \otimes \mathcal{W}_{2} \mathcal{W}_{D^{2}}\right) \times_{M \otimes \mathcal{W}_{D(2)}}\left(M \otimes \mathcal{W}_{D^{2}}\right) \\
& =M \otimes \mathcal{W}_{E} \rightarrow M \otimes \mathcal{W}_{C} \\
& =\left(M \otimes \mathcal{W}_{D^{2}}\right) \times_{M \otimes \mathcal{W}_{D(2)}}\left(M \otimes \mathcal{W}_{D^{2}}\right)
\end{aligned}
$$

and

$$
\left.\zeta_{2}^{-}: \underset{2}{\left(M \otimes \mathcal{W}_{D^{2}}\right.}\right) \times_{M \otimes \mathcal{W}_{D(2)}}\left(M \otimes \mathcal{W}_{D^{2}}\right) \rightarrow M \otimes \mathcal{W}_{D}
$$

in succession, which is in turn equivalent to

$$
\operatorname{id}_{M} \otimes \mathcal{W}_{d \in D \mapsto(0,0,-d, d) \in E}
$$

The morphism

$\zeta^{*_{1}-*_{3}}:\left(M \otimes \underset{1}{\mathcal{W}_{D^{2}}}\right) \times_{M \otimes} \mathcal{W}_{D(2)}\left(M \otimes \mathcal{W}_{D^{2}}\right) \times_{M} \otimes \mathcal{W}_{D(2)}\left(M \otimes \mathcal{W}_{D^{2}}\right) \rightarrow M \otimes \mathcal{W}_{D}$ is the composition of

$$
\begin{aligned}
& \operatorname{id}_{M} \otimes \mathcal{W}_{\left(d_{1}, d_{2}, d_{3}\right) \in D^{3}\{(1,3),(2,3)\} \mapsto\left(d_{1}, d_{2}, 0, d_{1} d_{2}-d_{3}\right) \in E} \\
& :\left(M \otimes \mathcal{W}_{D^{2}}\right) \times_{M \otimes \mathcal{W}_{D(2)}}\left(M \otimes \mathcal{W}_{D^{2}}\right) \times_{M} \otimes \mathcal{W}_{D(2)}\left(M \otimes \mathcal{W}_{D^{2}}\right) \\
& =M \otimes \mathcal{W}_{E} \rightarrow M \otimes \mathcal{W}_{C} \\
& =\left(M \otimes \mathcal{W}_{D^{2}}\right) \times_{M} \otimes \mathcal{W}_{D(2)}\left(M \otimes \mathcal{W}_{D^{2}}\right)
\end{aligned}
$$


and

$$
\left.\underset{3}{\left(M \otimes \mathcal{W}_{D^{2}}\right.}\right) \times_{M \otimes \mathcal{W}_{D(2)}}\left(M \otimes \mathcal{W}_{D^{2}}\right) \rightarrow M \otimes \mathcal{W}_{D}
$$

in succession, which is in turn equivalent to

$$
\operatorname{id}_{M} \otimes \mathcal{W}_{d \in D \mapsto(0,0,0,-d) \in E}
$$

Therefore

$$
\begin{gathered}
\zeta^{*_{2}-*_{1}}+\zeta^{*_{3}-*_{2}}+\zeta^{*_{1}-*_{3}} \\
:\left(M \otimes \mathcal{W}_{D^{2}}\right) \times_{M \otimes} \mathcal{W}_{D(2)} \\
\left.\underset{1}{(M \otimes} \mathcal{W}_{D^{2}}\right) \times_{M} \otimes \mathcal{W}_{D(2)} \\
\left(M \otimes \mathcal{W}_{D^{2}}\right) \rightarrow M \otimes \mathcal{W}_{D}
\end{gathered}
$$

is equivalent to

$$
\begin{aligned}
& \left(\operatorname{id}_{M} \otimes \mathcal{W}_{d \in D \mapsto(d, d, d) \in D(3)}\right) \circ\left(\operatorname{id}_{M} \otimes \mathcal{W}_{\left(d_{1}, d_{2}, d_{3}\right) \in D(3) \mapsto\left(0,0, d_{1}-d_{2}, d_{2}-d_{3}\right) \in E}\right) \\
& =\operatorname{id}_{M} \otimes\left(\mathcal{W}_{d \in D \mapsto(d, d, d) \in D(3)} \circ \mathcal{W}_{\left(d_{1}, d_{2}, d_{3}\right) \in D(3) \mapsto\left(0,0, d_{1}-d_{2}, d_{2}-d_{3}\right) \in E}\right) \\
& =\operatorname{id}_{M} \otimes \mathcal{W}_{d \in D \mapsto(0,0,0,0) \in E}
\end{aligned}
$$

This completes the proof.

\section{The Main Identity}

Proposition 10. The diagram

$$
\begin{aligned}
& \operatorname{id}_{M} \otimes \mathcal{W}_{\varphi_{1}^{3}}
\end{aligned}
$$

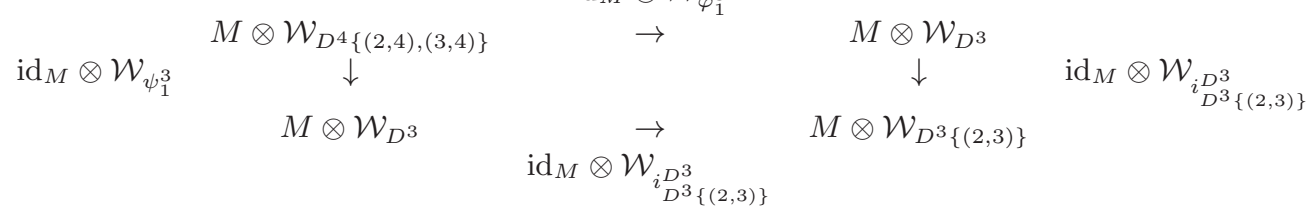

is a pullback diagram, where the assumptive mapping $\varphi_{1}^{3}: D^{3} \rightarrow D^{4}\{(2,4),(3$, 4) $\}$ is

$$
\left(d_{1}, d_{2}, d_{3}\right) \in D^{3} \mapsto\left(d_{1}, d_{2}, d_{3}, 0\right) \in D^{4}\{(2,4),(3,4)\}
$$

while the assumptive mapping $\psi_{1}^{3}: D^{3} \rightarrow D^{4}\{(2,4),(3,4)\}$ is

$$
\left(d_{1}, d_{2}, d_{3}\right) \in D^{3} \mapsto\left(d_{1}, d_{2}, d_{3}, d_{2} d_{3}\right) \in D^{4}\{(2,4),(3,4)\}
$$


Proof. This follows from the microlinearity of $M$ and the pullback diagram of Weil algebras

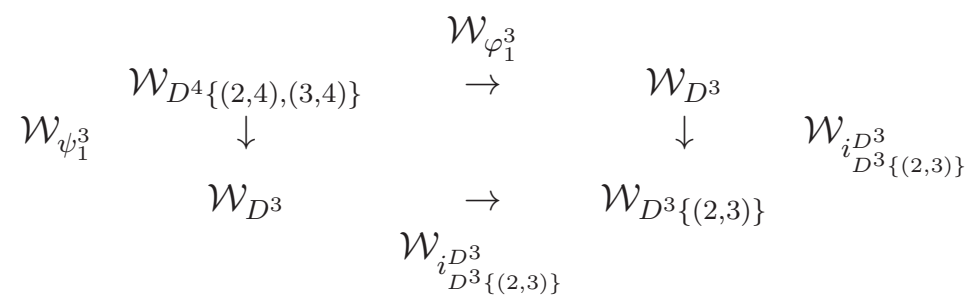

Corollary 11. We have

$$
\begin{aligned}
& \left(M \otimes \mathcal{W}_{D^{3}}\right) \times_{M \otimes \mathcal{W}_{D^{3}\{(2,3)\}}}\left(M \otimes \mathcal{W}_{D^{3}}\right) \\
& =M \otimes \mathcal{W}_{D^{4}\{(2,4),(3,4)\}}
\end{aligned}
$$

with the diagrams

$$
\begin{aligned}
& \underset{1}{\left.M \otimes \mathcal{W}_{D^{3}}\right) \times{ }_{M \otimes \mathcal{W}_{D^{3}\{(2,3)\}}}}\left(\begin{array}{ccc}
\left.M \otimes \mathcal{W}_{D^{3}}\right) & \rightarrow & M \otimes \mathcal{W}_{D^{3}} \\
\| & & 1
\end{array}\right. \\
& M \otimes \mathcal{W}_{D^{4}\{(2,4),(3,4)\}} \\
& \rightarrow \quad M \otimes \mathcal{W}_{D^{3}} \\
& \operatorname{id}_{M} \otimes \mathcal{W}_{\varphi_{1}^{3}}
\end{aligned}
$$

and

$$
\begin{array}{cccc}
\left(M \otimes \mathcal{W}_{D^{3}}\right) \times{ }_{M \otimes \mathcal{W}_{D^{3}\{(2,3)\}}}\left(M \otimes \mathcal{W}_{D^{3}}\right) & \rightarrow & M \otimes \mathcal{W}_{D^{3}} \\
& & & \|^{\|} \\
M \otimes \mathcal{W}_{D^{4}\{(2,4),(3,4)\}} & \rightarrow & M \otimes \mathcal{W}_{D^{3}} \\
& & \operatorname{id}_{M} \otimes \mathcal{W}_{\psi_{1}^{3}} &
\end{array}
$$

being commutative, where the unnamed arrows are canonical projections.

Notation 12. We will write

$$
\zeta^{\overline{1}}:\left(M \otimes \mathcal{W}_{D^{3}}\right) \times_{M \otimes \mathcal{W}_{D^{3}\{(2,3)\}}}\left(M \otimes \mathcal{W}_{D^{3}}\right) \rightarrow M \otimes \mathcal{W}_{D^{2}}
$$

for the morphism

$$
\operatorname{id}_{M} \otimes \mathcal{W}_{\left(d_{1}, d_{2}\right) \in D^{2} \mapsto\left(d_{1}, 0,0, d_{2}\right) \in D^{4}\{(2,4),(3,4)\}}
$$




$$
\begin{aligned}
& :\left(M \otimes \mathcal{W}_{D^{3}}\right) \times_{M \otimes \mathcal{W}_{D^{3}\{(2,3)\}}}\left(M \otimes \mathcal{W}_{D^{3}}\right) \\
& =M \otimes \mathcal{W}_{D^{4}\{(2,4),(3,4)\}} \\
& \rightarrow M \otimes \mathcal{W}_{D^{2}}
\end{aligned}
$$

Proposition 13. The diagram

$$
\begin{array}{ccc}
M \otimes \mathcal{W}_{D^{4}\{(1,4),(3,4)\}} & \stackrel{\operatorname{id}_{M} \otimes \mathcal{W}_{\varphi_{2}^{3}}}{\longrightarrow} & M \otimes \mathcal{W}_{D^{3}} \\
\operatorname{id}_{M} \otimes \mathcal{W}_{\psi_{2}^{3}} \downarrow & & \downarrow \operatorname{id}_{M} \otimes \mathcal{W}_{i_{D^{3}\{(1,3)\}}} \\
M \otimes \mathcal{W}_{D^{3}} & \stackrel{\operatorname{id}_{M} \otimes \mathcal{W}_{i_{D^{3}\{(1,3)\}}}}{\longrightarrow} & M \otimes \mathcal{W}_{D^{3}\{(1,3)\}}
\end{array}
$$

is a pullback diagram, where the assumptive mapping $\varphi_{2}^{3}: D^{3} \rightarrow D^{4}\{(1,4),(3$, $4)\}$ is

$$
\left(d_{1}, d_{2}, d_{3}\right) \in D^{3} \mapsto\left(d_{1}, d_{2}, d_{3}, 0\right) \in D^{4}\{(1,4),(3,4)\}
$$

while the assumptive mapping $\psi_{2}^{3}: D^{3} \rightarrow D^{4}\{(1,4),(3,4)\}$ is

$$
\left(d_{1}, d_{2}, d_{3}\right) \in D^{3} \mapsto\left(d_{1}, d_{2}, d_{3}, d_{1} d_{3}\right) \in D^{4}\{(1,4),(3,4)\}
$$

Proof. This follows from the microlinearity of $M$ and the pullback diagram of Weil algebras

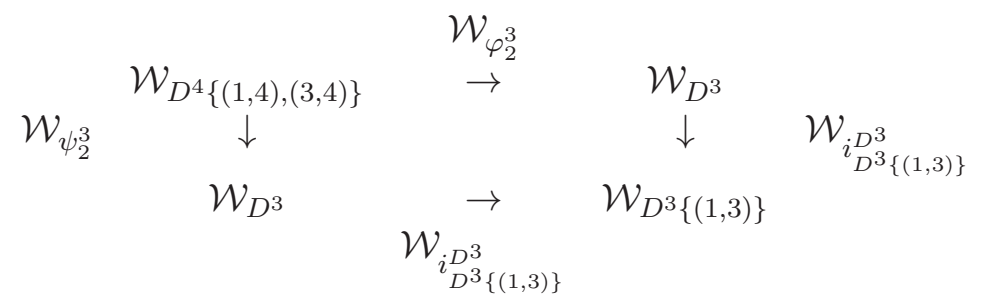

Corollary 14. We have

$$
\begin{aligned}
& \left(M \otimes \mathcal{W}_{D^{3}}\right) \times_{M \otimes \mathcal{W}_{D^{3}\{(1,3)\}}}\left(M \otimes \mathcal{W}_{D^{3}}\right) \\
& =M \otimes \mathcal{W}_{D^{4}\{(1,4),(3,4)\}}
\end{aligned}
$$

with the diagrams

$$
\begin{array}{cccc}
\left(M \otimes \mathcal{W}_{D^{3}}\right) \times_{M \otimes \mathcal{W}_{D^{3}\{(1,3)\}}}\left(M \otimes \mathcal{W}_{D^{3}}\right) & \rightarrow & M \otimes \mathcal{W}_{D^{3}} \\
\| & & & \| \\
M \otimes \mathcal{W}_{D^{4}\{(1,4),(3,4)\}} & \rightarrow & M \otimes \mathcal{W}_{D^{3}} \\
& & \operatorname{id}_{M} \otimes \mathcal{W}_{\varphi_{2}^{3}} &
\end{array}
$$


and

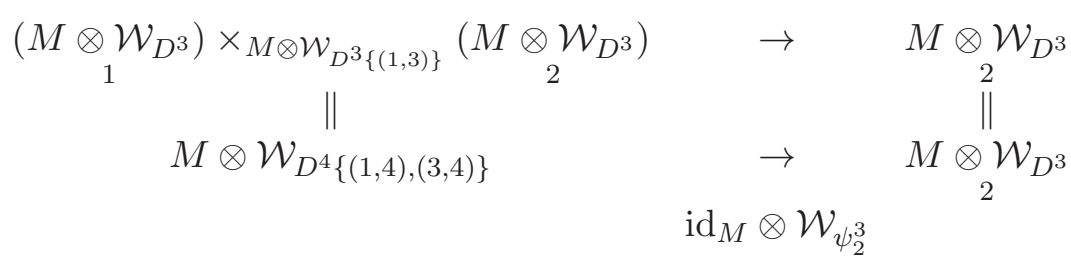

being commutative, where unnamed arrows are canonical projections.

Notation 15. We will write

$$
\zeta^{\overline{2}}:\left(M \otimes \mathcal{W}_{D^{3}}\right) \times_{M \otimes \mathcal{W}_{D^{3}\{(1,3)\}}}\left(M \otimes \mathcal{W}_{D^{3}}\right) \rightarrow M \otimes \mathcal{W}_{D^{2}}
$$

for the morphism

$$
\begin{aligned}
& \operatorname{id}_{M} \otimes \mathcal{W}_{\left(d_{1}, d_{2}\right) \in D^{2} \mapsto\left(0, d_{1}, 0, d_{2}\right) \in D^{4}\{(1,4),(3,4)\}}\left(M \otimes \mathcal{W}_{D^{3}}\right) \\
& :\left(M \otimes \mathcal{W}_{\left.D^{3}\right) \times_{M} \otimes \mathcal{W}_{D^{3}\{(1,3)\}}(M}\right. \\
& =M \otimes \mathcal{W}_{D^{4}\{(1,4),(3,4)\}} \\
& \rightarrow M \otimes \mathcal{W}_{D^{2}}
\end{aligned}
$$

Proposition 16. The diagram

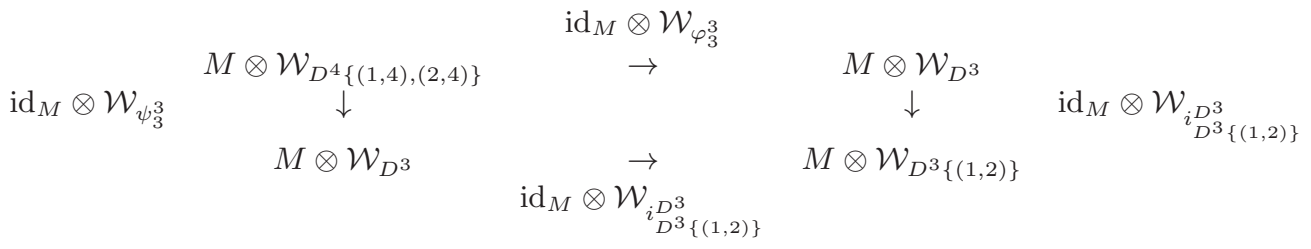

is a pullback diagram, where the assumptive mapping $\varphi_{3}^{3}: D^{3} \rightarrow D^{4}\{(1,4),(2,4)\}$ is

$$
\left(d_{1}, d_{2}, d_{3}\right) \in D^{3} \mapsto\left(d_{1}, d_{2}, d_{3}, 0\right) \in D^{4}\{(1,4),(2,4)\}
$$

while the assumptive mapping $\psi_{3}^{3}: D^{3} \rightarrow D^{4}\{(1,4),(2,4)\}$ is

$$
\left(d_{1}, d_{2}, d_{3}\right) \in D^{3} \mapsto\left(d_{1}, d_{2}, d_{3}, d_{1} d_{2}\right) \in D^{4}\{(1,4),(2,4)\}
$$

Proof. This follows from the microlinearity of $M$ and the pullback diagram of Weil algebras

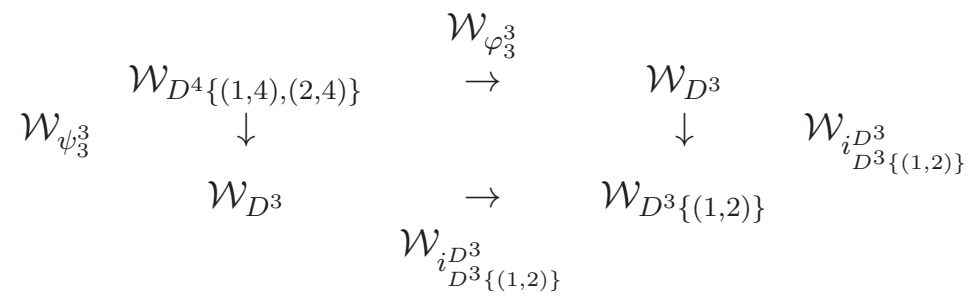


Corollary 17. We have

$$
\begin{aligned}
& \left(M \otimes \mathcal{W}_{D^{3}}\right) \times_{M \otimes \mathcal{W}_{D^{3}\{(1,2)\}}}\left(M \otimes \mathcal{W}_{D^{3}}\right) \\
& =M \otimes \mathcal{W}_{D^{4}\{(1,4),(2,4)\}}
\end{aligned}
$$

with the diagrams

$$
\begin{array}{cccc}
\left(M \otimes \mathcal{W}_{D^{3}}\right) \times{ }_{M \otimes \mathcal{W}_{D^{3}\{(1,2)\}}}\left(M \otimes \mathcal{W}_{D^{3}}\right) & \rightarrow & M \otimes \mathcal{W}_{D^{3}} \\
M \otimes \mathcal{W}_{D^{4}\{(1,4),(2,4)\}} & & \rightarrow & M \otimes \mathcal{W}_{D^{3}} \\
& & \operatorname{id}_{M} \otimes \mathcal{W}_{\varphi_{3}^{3}} &
\end{array}
$$

and

$$
\operatorname{id}_{M} \otimes \mathcal{W}_{\psi_{3}^{3}}
$$

being commutative, where unnamed arrows are canonical projections.

Notation 18. We will write

$$
\zeta^{\overline{3}}:\left(M \otimes \mathcal{W}_{D^{3}}\right) \times_{M \otimes \mathcal{W}_{D^{3}\{(1,2)\}}}\left(M \otimes \mathcal{W}_{D^{3}}\right) \rightarrow M \otimes \mathcal{W}_{D^{2}}
$$

for the morphism

$$
\begin{aligned}
& \operatorname{id}_{M} \otimes \mathcal{W}_{\left(d_{1}, d_{2}\right) \in D^{2} \mapsto\left(0,0, d_{1}, d_{2}\right) \in D^{4}\{(1,4),(3,4)\}} \\
& :\left(M \otimes \mathcal{W}_{D^{3}}\right) \times_{M \otimes \mathcal{W}_{D^{3}\{(1,2)\}}}\left(M \otimes \mathcal{W}_{D^{3}}\right) \\
& =M \otimes \mathcal{W}_{D^{4}\{(1,4),(2,4)\}} \\
& \rightarrow M \otimes \mathcal{W}_{D^{2}}
\end{aligned}
$$

Notation 19. We will write $i_{14}^{1}, i_{24}^{2}$ and $i_{34}^{3}$ for the assumptive mappings

$$
\begin{gathered}
\left(d_{1}, d_{2}\right) \in D(2) \mapsto\left(d_{1}, 0,0, d_{2}\right) \in D^{4}\{(2,4),(3,4)\}, \\
\left(d_{1}, d_{2}\right) \in D(2) \mapsto\left(0, d_{1}, 0, d_{2}\right) \in D^{4}\{(1,4),(3,4)\}
\end{gathered}
$$

and

$$
\left(d_{1}, d_{2}\right) \in D(2) \mapsto\left(0,0, d_{1}, d_{2}\right) \in D^{4}\{(1,4),(2,4)\}
$$

respectively. 
Proposition 20. The diagram

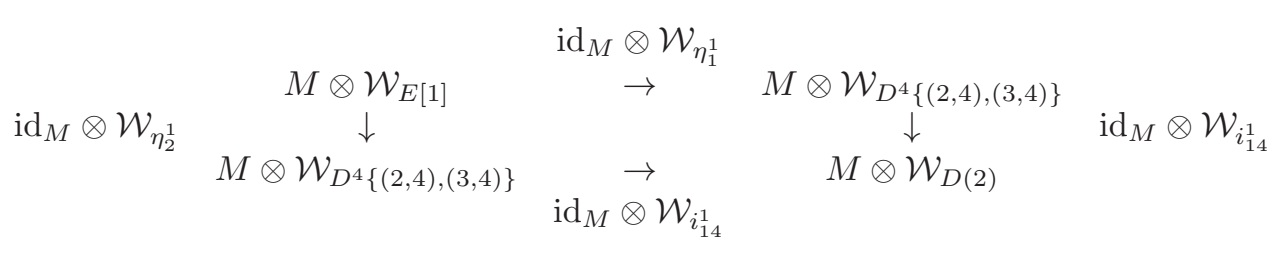

is a pullback, where the assumptive object $E[1]$ is

$$
\begin{aligned}
& D^{7}\{(2,6),(3,6),(4,6),(5,6),(1,7),(2,7),(3,7),(4,7),(5,7),(6,7),(2,4), \\
& (2,5),(3,4),(3,5)\},
\end{aligned}
$$

the assumptive mapping

$$
\eta_{1}^{1}: D^{4}\{(2,4),(3,4)\} \rightarrow E[1]
$$

is

$$
\begin{aligned}
\left(d_{1}, d_{2}, d_{3}, d_{4}\right) & \in D^{4}\{(2,4),(3,4)\} \mapsto \\
\left(d_{1}, d_{2}, d_{3}, 0,0, d_{4}, 0\right) & \in E[1],
\end{aligned}
$$

and the assumptive mapping

$$
\eta_{2}^{1}: D^{4}\{(2,4),(3,4)\} \rightarrow E[1]
$$

is

$$
\begin{aligned}
\left(d_{1}, d_{2}, d_{3}, d_{4}\right) & \in D^{4}\{(2,4),(3,4)\} \mapsto \\
\left(d_{1}, 0,0, d_{2}, d_{3}, d_{4}, d_{1} d_{4}\right) & \in E[1] .
\end{aligned}
$$

Proof. This follows from the microlinearity of $M$ and the pullback diagram of Weil algebras

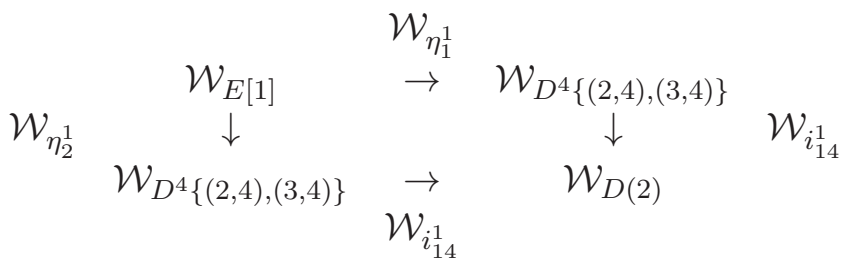


Notation 21. We will write $\iota_{1}^{1}, \iota_{2}^{1}, \iota_{3}^{1}$ and $\iota_{4}^{1}$ for the assumptive mappings $\eta_{1}^{1} \circ \varphi_{1}^{3}, \eta_{1}^{1} \circ \psi_{1}^{3}, \eta_{2}^{1} \circ \varphi_{1}^{3}$ and $\eta_{2}^{1} \circ \psi_{1}^{3}$ respectively. That is to say, we have

$$
\begin{aligned}
& \iota_{1}^{1}:\left(d_{1}, d_{2}, d_{3}\right) \in D^{3} \mapsto\left(d_{1}, d_{2}, d_{3}, 0,0,0,0\right) \in E[1] \\
& \iota_{2}^{1}:\left(d_{1}, d_{2}, d_{3}\right) \in D^{3} \mapsto\left(d_{1}, d_{2}, d_{3}, 0,0, d_{2} d_{3}, 0\right) \in E[1] \\
& \iota_{3}^{1}:\left(d_{1}, d_{2}, d_{3}\right) \in D^{3} \mapsto\left(d_{1}, 0,0, d_{2}, d_{3}, 0,0\right) \in E[1] \\
& \iota_{4}^{1}:\left(d_{1}, d_{2}, d_{3}\right) \in D^{3} \mapsto\left(d_{1}, 0,0, d_{2}, d_{3}, d_{2} d_{3}, d_{1} d_{2} d_{3}\right) \in E[1]
\end{aligned}
$$

Corollary 22. We have

$$
\begin{aligned}
& \left(\begin{array}{c}
\left(M \otimes \mathcal{W}_{D^{3}}\right) \\
\times_{M \otimes \mathcal{W}_{D^{3}\{(2,3)\}}} \\
\left(M \otimes \mathcal{W}_{D^{3}}\right)
\end{array}\right)^{\times_{M \otimes} \otimes \mathcal{W}_{D(2)}}\left(\begin{array}{c}
\left(M \otimes \mathcal{W}_{D^{3}}\right) \\
\times_{M \otimes \mathcal{W}_{D^{3}\{(2,3)\}}} \\
\left(M \otimes \mathcal{W}_{D^{3}}\right)
\end{array}\right) \\
& =M \otimes \mathcal{W}_{E[1]}
\end{aligned}
$$

with the diagrams

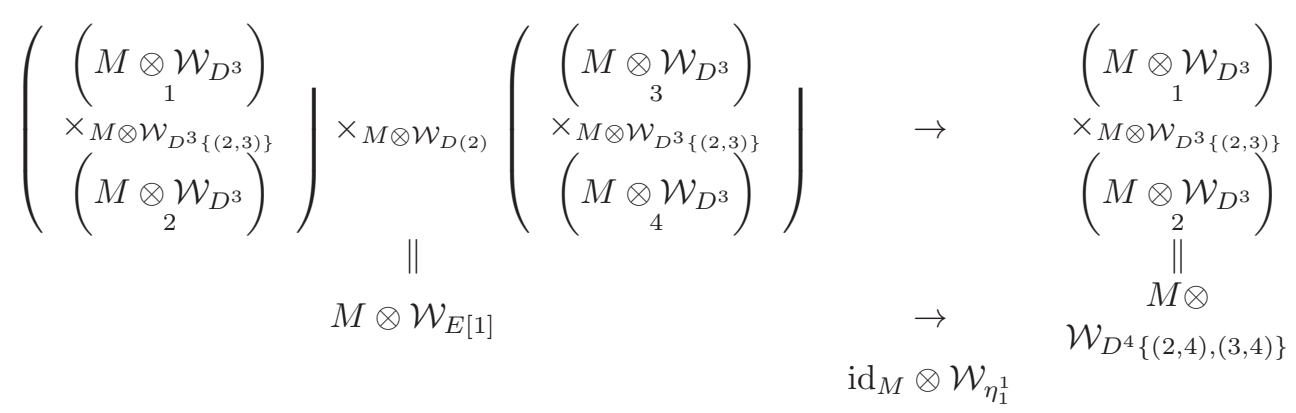

and

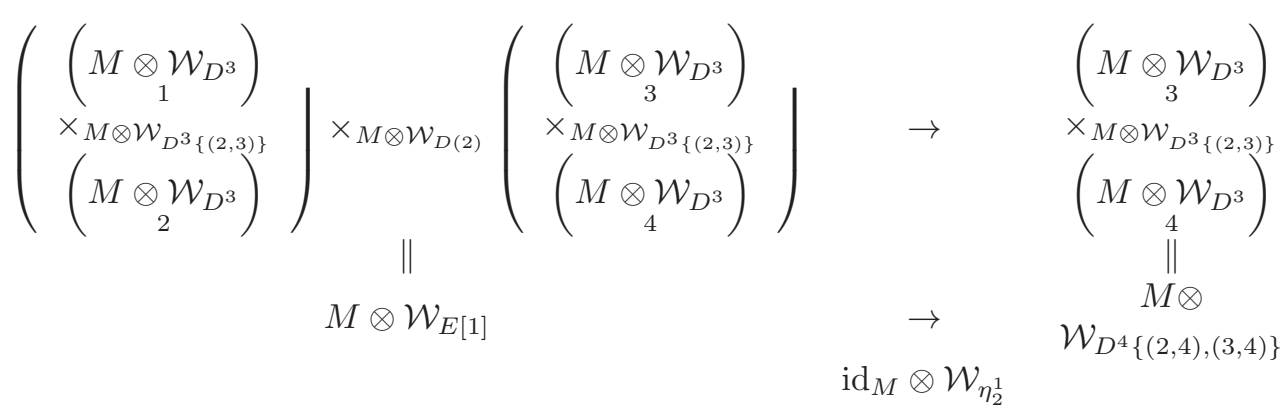

being commutative, where unnamed arrows are canonical projections. 
Proposition 23. The diagram

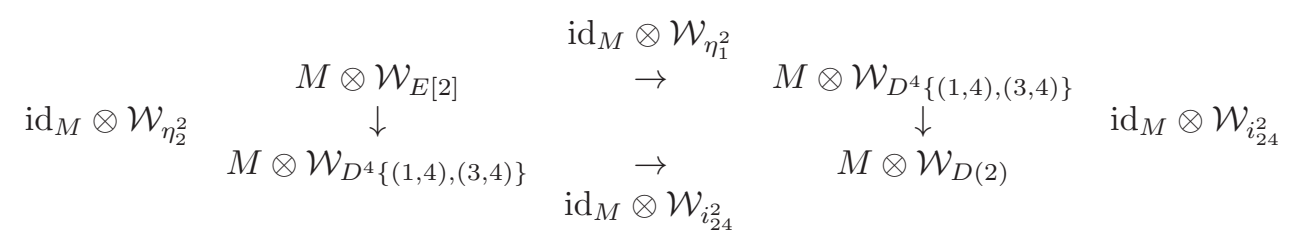

is a pullback, where the assumptive object $E[2]$ is

$$
\begin{aligned}
& D^{7}\{(1,6),(3,6),(4,6),(5,6),(1,7),(2,7),(3,7),(4,7),(5,7),(6,7),(1,4), \\
& (1,5),(3,4),(3,5)\}
\end{aligned}
$$

the assumptive mapping $\left.\eta_{1}^{2}: D^{4}\{(1,4),(3,4)\}\right\} \rightarrow E[2]$ is

$$
\begin{aligned}
\left(d_{1}, d_{2}, d_{3}, d_{4}\right) & \in D^{4}\{(1,4),(3,4)\} \mapsto \\
\left(d_{1}, d_{2}, d_{3}, 0,0, d_{4}, 0\right) & \in E[2],
\end{aligned}
$$

and the assumptive mapping $\eta_{2}^{2}: D^{4}\{(1,4),(3,4)\} \rightarrow E[2]$ is

$$
\begin{aligned}
\left(d_{1}, d_{2}, d_{3}, d_{4}\right) & \in D^{4}\{(1,4),(3,4)\} \mapsto \\
\left(0, d_{2}, 0, d_{1}, d_{3}, d_{4}, d_{2} d_{4}\right) & \in E[2] .
\end{aligned}
$$

Proof. This follows from the microlinearity of $M$ and the pullback diagram of Weil algebras

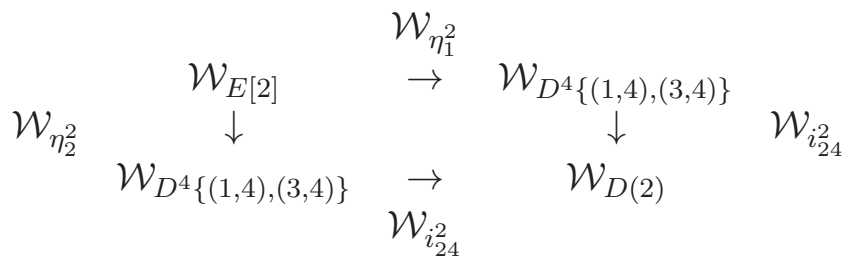

Notation 24. We will write $\iota_{1}^{2}, \iota_{2}^{2}, \iota_{3}^{2}$ and $\iota_{4}^{2}$ for the assumptive mappings $\eta_{1}^{2} \circ \varphi_{2}^{3}, \eta_{1}^{2} \circ \psi_{2}^{3}, \eta_{2}^{2} \circ \varphi_{2}^{3}$ and $\eta_{2}^{2} \circ \psi_{2}^{3}$ respectively. That is to say, we have

$$
\begin{aligned}
& \iota_{1}^{2}:\left(d_{1}, d_{2}, d_{3}\right) \in D^{3} \mapsto\left(d_{1}, d_{2}, d_{3}, 0,0,0,0\right) \in E[2] \\
& \iota_{2}^{2}:\left(d_{1}, d_{2}, d_{3}\right) \in D^{3} \mapsto\left(d_{1}, d_{2}, d_{3}, 0,0, d_{2} d_{3}, 0\right) \in E[2] \\
& \iota_{3}^{2}:\left(d_{1}, d_{2}, d_{3}\right) \in D^{3} \mapsto\left(0, d_{2}, 0, d_{3}, d_{1}, 0,0\right) \in E[2] \\
& \iota_{4}^{2}:\left(d_{1}, d_{2}, d_{3}\right) \in D^{3} \mapsto\left(0, d_{2}, 0, d_{3}, d_{1}, d_{1} d_{3}, d_{1} d_{2} d_{3}\right) \in E[2]
\end{aligned}
$$


Corollary 25. We have

$$
\begin{aligned}
& \left(\begin{array}{c}
\left(M \otimes \mathcal{W}_{D^{3}}\right) \\
\times_{M \otimes \mathcal{W}_{D^{3}\{(1,3)\}}} \\
\left(M \otimes \mathcal{W}_{D^{3}}\right)
\end{array}\right)^{\times_{M \otimes \mathcal{W}_{D(2)}}}\left(\begin{array}{c}
\left(M \otimes \mathcal{W}_{D^{3}}\right) \\
\times_{M \otimes \mathcal{W}_{D^{3}\{(1,3)\}}} \\
\left(M \otimes \mathcal{W}_{D^{3}}\right)
\end{array}\right) \\
& =M \otimes \mathcal{W}_{E[2]}
\end{aligned}
$$

with the diagrams

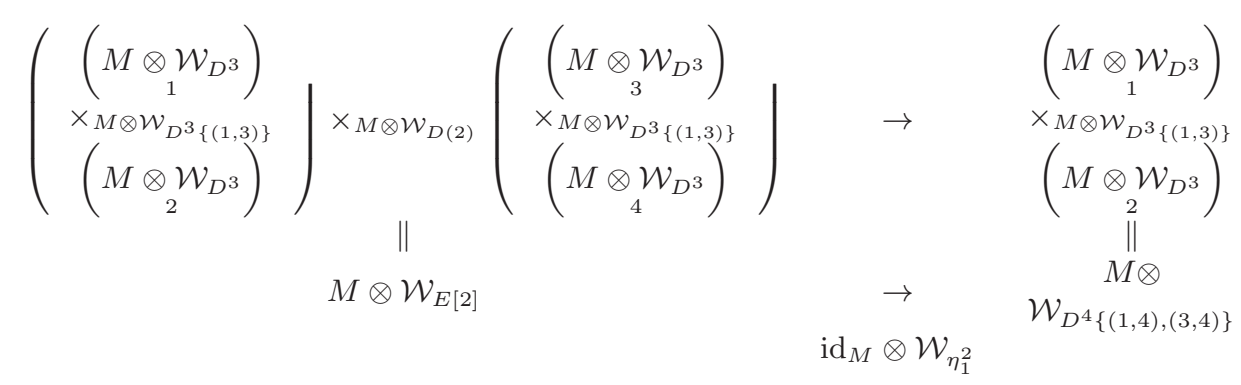

and

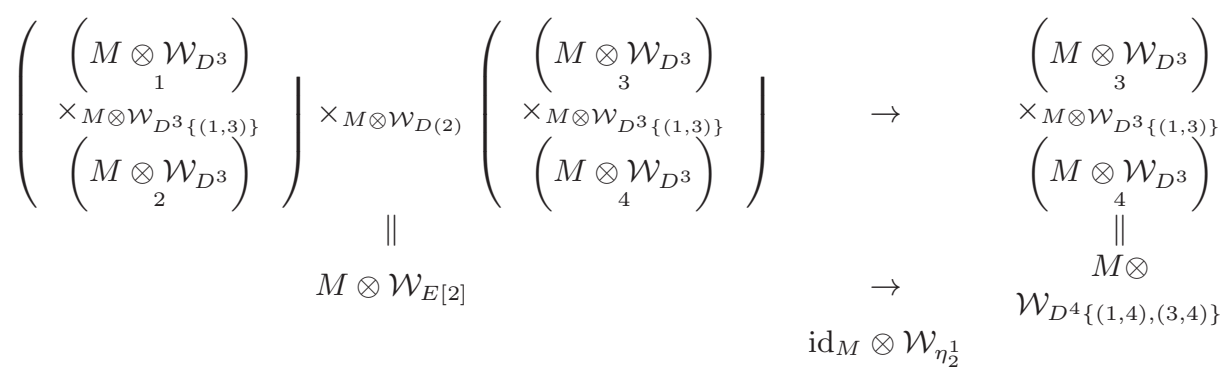

being commutative, where unnamed arrows are the canonical projections.

Proposition 26. The diagram

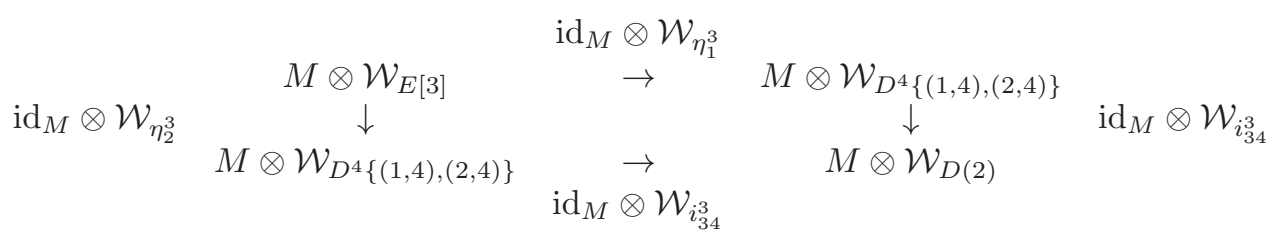

is a pullback, where the assumptive object $E[3]$ is

$$
\begin{aligned}
& D^{7}\{(1,6),(2,6),(4,6),(5,6),(1,7),(2,7),(3,7),(4,7),(5,7),(6,7),(1,4), \\
& (1,5),(2,4),(2,5)\}\},
\end{aligned}
$$


the assumptive mapping $\left.\eta_{1}^{3}: D^{4}\{(1,4),(2,4)\}\right\} \rightarrow E[3]$ is

$$
\begin{aligned}
\left(d_{1}, d_{2}, d_{3}, d_{4}\right) & \in D^{4}\{(1,4),(2,4)\} \mapsto \\
\left(d_{1}, d_{2}, d_{3}, 0,0, d_{4}, 0\right) & \in E[3],
\end{aligned}
$$

and the assumptive mapping $\eta_{2}^{3}: D^{4}\{(1,4),(3,4)\} \rightarrow E[3]$ is

$$
\begin{aligned}
\left(d_{1}, d_{2}, d_{3}, d_{4}\right) & \in D^{4}\{(1,4),(2,4)\} \mapsto \\
\left(0,0, d_{3}, d_{1}, d_{2}, d_{4}, d_{3} d_{4}\right) & \in E[3] .
\end{aligned}
$$

Proof. This follows from the microlinearity of $M$ and the pullback diagram of Weil algebras

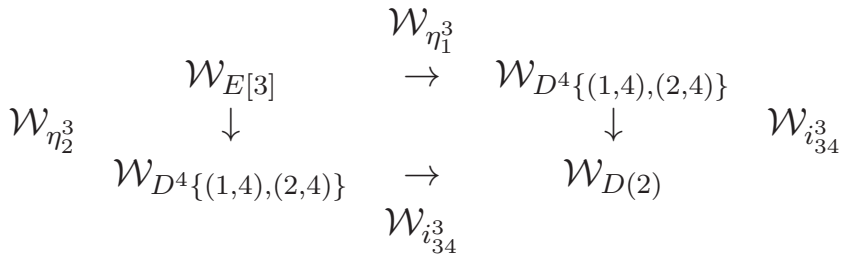

Notation 27. We will write $\iota_{1}^{3}, \iota_{2}^{3}, \iota_{3}^{3}$ and $\iota_{4}^{3}$ for the assumptive mappings $\eta_{1}^{3} \circ \varphi_{3}^{3}, \eta_{1}^{3} \circ \psi_{3}^{3}, \eta_{2}^{3} \circ \varphi_{3}^{3}$ and $\eta_{2}^{3} \circ \psi_{3}^{3}$ respectively. That is to say, we have

$$
\begin{aligned}
& \iota_{1}^{3}:\left(d_{1}, d_{2}, d_{3}\right) \in D^{3} \mapsto\left(d_{1}, d_{2}, d_{3}, 0,0,0,0\right) \in E[3] \\
& \iota_{2}^{3}:\left(d_{1}, d_{2}, d_{3}\right) \in D^{3} \mapsto\left(d_{1}, d_{2}, d_{3}, 0,0, d_{1} d_{2}, 0\right) \in E[3] \\
& \iota_{3}^{3}:\left(d_{1}, d_{2}, d_{3}\right) \in D^{3} \mapsto\left(0,0, d_{3}, d_{1}, d_{2}, 0,0\right) \in E[3] \\
& \iota_{4}^{3}:\left(d_{1}, d_{2}, d_{3}\right) \in D^{3} \mapsto\left(0,0, d_{3}, d_{1}, d_{2}, d_{1} d_{2}, d_{1} d_{2} d_{3}\right) \in E[3]
\end{aligned}
$$

Corollary 28. We have

$$
\begin{aligned}
& \left(\begin{array}{c}
\left(M \otimes \mathcal{W}_{D^{3}}\right) \\
\times_{M \otimes \mathcal{W}_{D^{3}\{(1,2)\}}} \\
\left(M \otimes \mathcal{W}_{D^{3}}\right)
\end{array}\right)^{\times_{M \otimes \mathcal{W}_{D(2)}}}\left(\begin{array}{c}
\left(M \otimes \mathcal{W}_{D^{3}}\right) \\
\times_{M \otimes \mathcal{W}_{D^{3}\{(1,2)\}}} \\
\left(M \otimes \mathcal{W}_{D^{3}}\right)
\end{array}\right) \\
& =M \otimes \mathcal{W}_{E[3]}
\end{aligned}
$$


with the diagrams

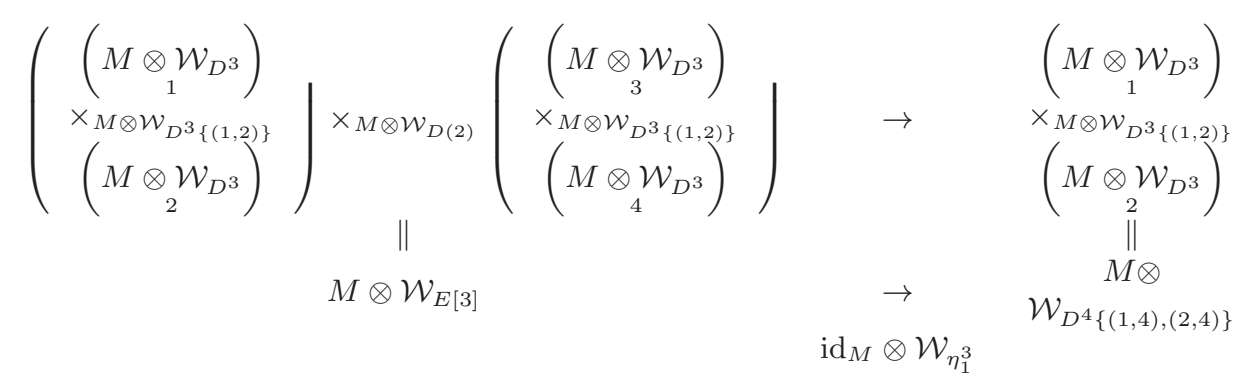

and

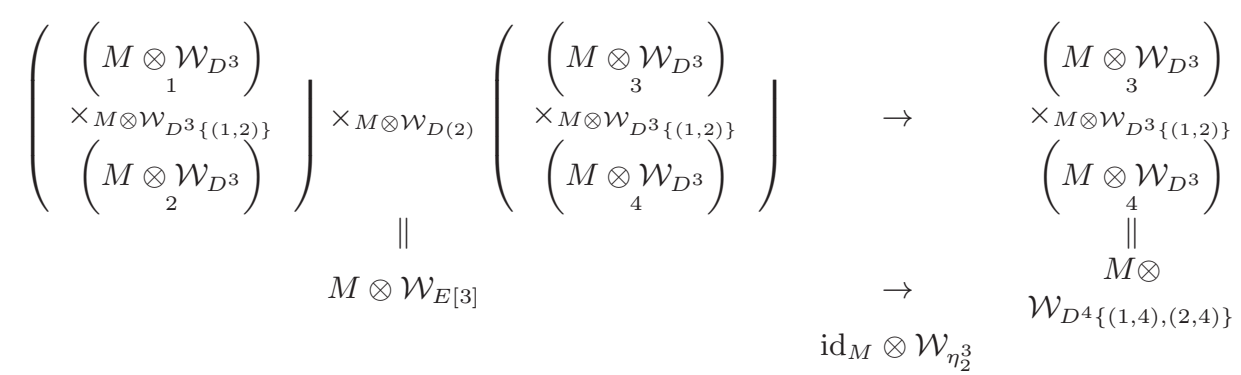

being commutative, where unnamed arrows are the canonical projections. 
Now we come to the crucial step in the proof of the general Jacobi identity.

Notation 29. A limit of the diagram

$$
\begin{aligned}
& \left(\begin{array}{c}
\left(M \otimes \mathcal{W}_{D^{3}}\right) \\
321 \\
\times M \otimes \mathcal{W}_{D^{3}\{(2,3)\}} \\
\left(M \otimes \mathcal{W}_{D^{3}}\right) \\
231
\end{array}\right) \\
& \times_{M \otimes \mathcal{W}_{D(2)}} \\
& \left(\begin{array}{c}
\left(M \otimes \mathcal{W}_{D^{3}}\right) \\
132 \\
\times_{M \otimes \mathcal{W}_{D^{3}\{(2,3)\}}} \\
\left(M \otimes \mathcal{W}_{D^{3}}\right) \\
123
\end{array}\right) \\
& \left(M \otimes \mathcal{W}_{D^{3}}\right) \\
& 231 \\
& \times_{M} \\
& \left(M \otimes \mathcal{W}_{D^{3}}\right) \\
& 132 \\
& \left(\begin{array}{c}
\left(M \otimes \mathcal{W}_{D^{3}}\right) \\
132 \\
\times M \otimes \mathcal{W}_{D^{3}\{(1,3)\}} \\
\left(M \otimes \mathcal{W}_{D^{3}}\right) \\
312
\end{array}\right) \\
& \times_{M \otimes \mathcal{W}_{D(2)}} \\
& \left(\begin{array}{c}
\left(M \otimes \mathcal{W}_{D^{3}}\right) \\
213 \\
\times_{M \otimes \mathcal{W}_{D^{3}\{(1,3)\}}} \\
\left(M \otimes \mathcal{W}_{D^{3}}\right) \\
231
\end{array}\right) \\
& \left(M \otimes \mathcal{W}_{D^{3}}\right) \\
& 123 \\
& \times_{M} \\
& \left(M \otimes \mathcal{W}_{D^{3}}\right) \\
& { }^{321} \\
& \left(\begin{array}{c}
\left(M \otimes \mathcal{W}_{D^{3}}\right) \\
213 \\
\times M \otimes \mathcal{W}_{D^{3}\{(1,2)\}} \\
\left(M \otimes \mathcal{W}_{D^{3}}\right) \\
123
\end{array}\right) \\
& \times_{M \otimes \mathcal{W}_{D(2)}} \\
& \left(\begin{array}{c}
\left(M \otimes \mathcal{W}_{D^{3}}\right) \\
321 \\
\times M \otimes \mathcal{W}_{D^{3}\{(1,2)\}} \\
\left(M \otimes \mathcal{W}_{D^{3}}\right) \\
312
\end{array}\right) \\
& \left(M \otimes \mathcal{W}_{D^{3}}\right) \\
& 312 \\
& \times_{M} \\
& \left(M \otimes \mathcal{W}_{D^{3}}\right) \\
& 213
\end{aligned}
$$


with every arrow being the canonical projection is denoted by

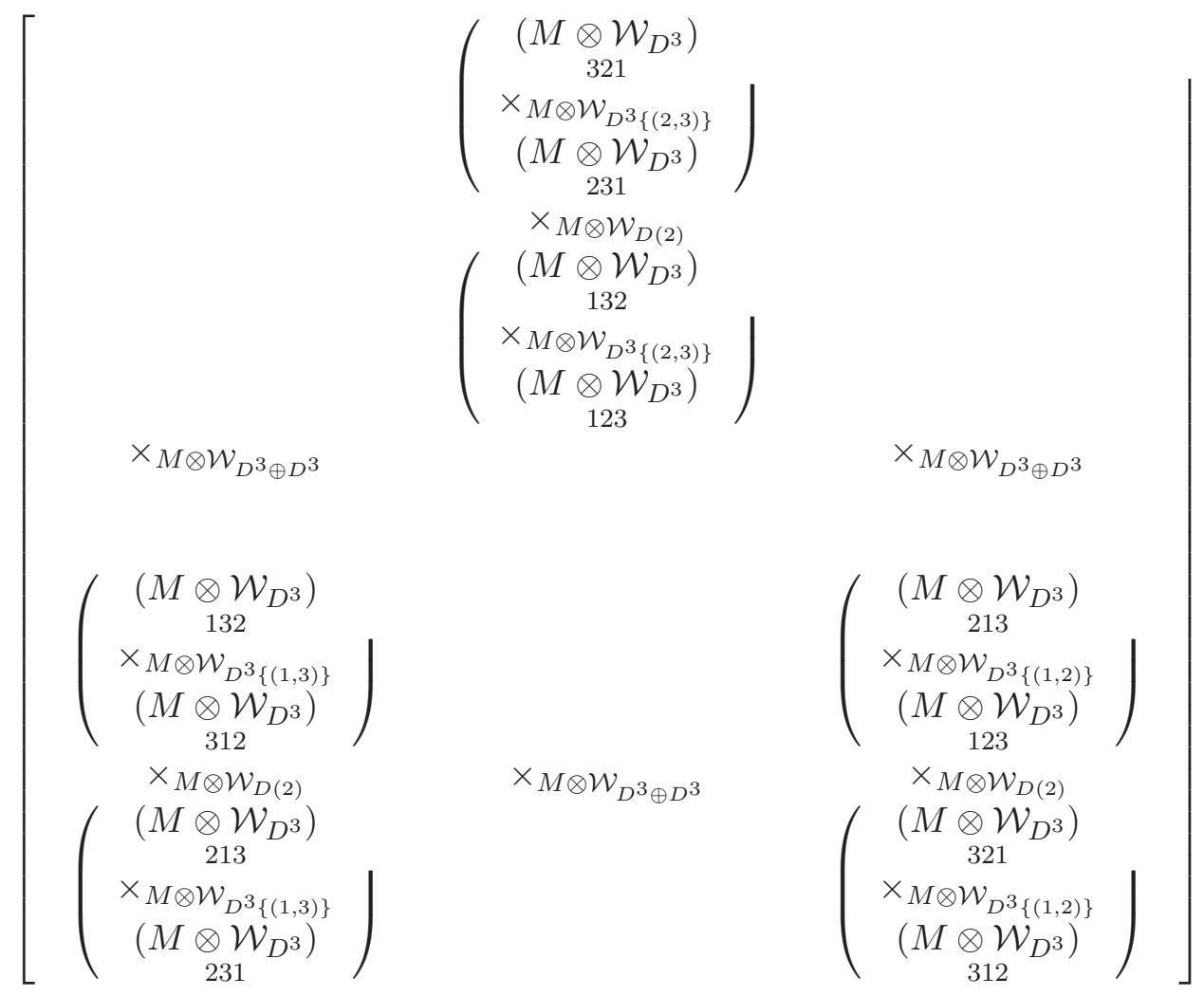

We can compute the above limit.

Theorem 30. The diagram

$$
\begin{aligned}
& \operatorname{id}_{M} \otimes \mathcal{W}_{h_{12}^{1}} \quad \begin{array}{cccc}
M \otimes \mathcal{W}_{E[1]} & & & \operatorname{id}_{M} \otimes \mathcal{W}_{h_{31}^{1}} \\
& \swarrow & \searrow &
\end{array}
\end{aligned}
$$

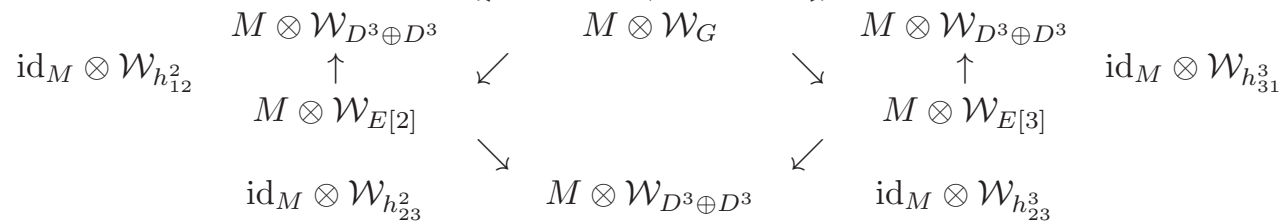

is a limit diagram with the three unnamed arrows being

$$
\begin{aligned}
& \operatorname{id}_{M} \otimes \mathcal{W}_{k_{1}}: M \otimes \mathcal{W}_{G} \rightarrow M \otimes \mathcal{W}_{E[1]} \\
& \operatorname{id}_{M} \otimes \mathcal{W}_{k_{2}}: M \otimes \mathcal{W}_{G} \rightarrow M \otimes \mathcal{W}_{E[2]} \\
& \operatorname{id}_{M} \otimes \mathcal{W}_{k_{3}}: M \otimes \mathcal{W}_{G} \rightarrow M \otimes \mathcal{W}_{E[3]}
\end{aligned}
$$


where the assumptive object $G$ is

$$
\begin{aligned}
& D^{8}\{(2,4),(3,4),(1,5),(3,5),(1,6),(2,6),(4,5),(4,6),(5,6),(1,7),(2,7),(3,7), \\
& (4,7),(5,7),(6,7),(1,8),(2,8),(3,8),(4,8),(5,8),(6,8),(7,8)\}
\end{aligned}
$$

the assumptive mapping $k_{1}: E[1] \rightarrow G$ is

$$
\begin{aligned}
\left(d_{1}, d_{2}, d_{3}, d_{4}, d_{5}, d_{6}, d_{7}\right) \in E[1] & \mapsto \\
\left(d_{1}, d_{2}+d_{4}, d_{3}+d_{5}, d_{6}-d_{2} d_{3}-d_{4} d_{5},-d_{1} d_{5}, d_{1} d_{4}, d_{7}+d_{1} d_{2} d_{3}, d_{1} d_{2} d_{3}\right) & \in G,
\end{aligned}
$$

the assumptive mapping $k_{2}: E[2] \rightarrow G$ is

$$
\begin{aligned}
\left(d_{1}, d_{2}, d_{3}, d_{4}, d_{5}, d_{6}, d_{7}\right) & \in E[2] \mapsto \\
\left(d_{1}+d_{5}, d_{2}, d_{3}+d_{4},-d_{2} d_{3}, d_{6}-d_{1} d_{3}-d_{4} d_{5}, d_{1} d_{2}, d_{2} d_{4} d_{5}, d_{7}\right) & \in G,
\end{aligned}
$$

the assumptive mapping $k_{3}: E[3] \rightarrow G$ is

$$
\begin{aligned}
\left(d_{1}, d_{2}, d_{3}, d_{4}, d_{5}, d_{6}, d_{7}\right) & \in E[3] \mapsto \\
\left(d_{1}+d_{4}, d_{2}+d_{5}, d_{3},-d_{4} d_{5},-d_{1} d_{3}, d_{6},-d_{7},-d_{7}+d_{3} d_{4} d_{5}\right) & \in G,
\end{aligned}
$$

the assumptive mapping $h_{12}^{1}$ is

$$
\iota_{2}^{1} \oplus \iota_{3}^{1},
$$

the assumptive mapping $h_{12}^{2}$ is

$$
\iota_{4}^{2} \oplus \iota_{1}^{2},
$$

the assumptive mapping $h_{23}^{2}$ is

$$
\iota_{2}^{2} \oplus \iota_{3}^{2}
$$

the assumptive mapping $h_{23}^{3}$ is

$$
\iota_{4}^{3} \oplus \iota_{1}^{3},
$$

the assumptive mapping $h_{31}^{3}$ is

$$
\iota_{2}^{3} \oplus \iota_{3}^{3},
$$

and the assumptive mapping $h_{31}^{1}$ is

$$
\iota_{4}^{1} \oplus \iota_{1}^{2}
$$


Corollary 31. We have

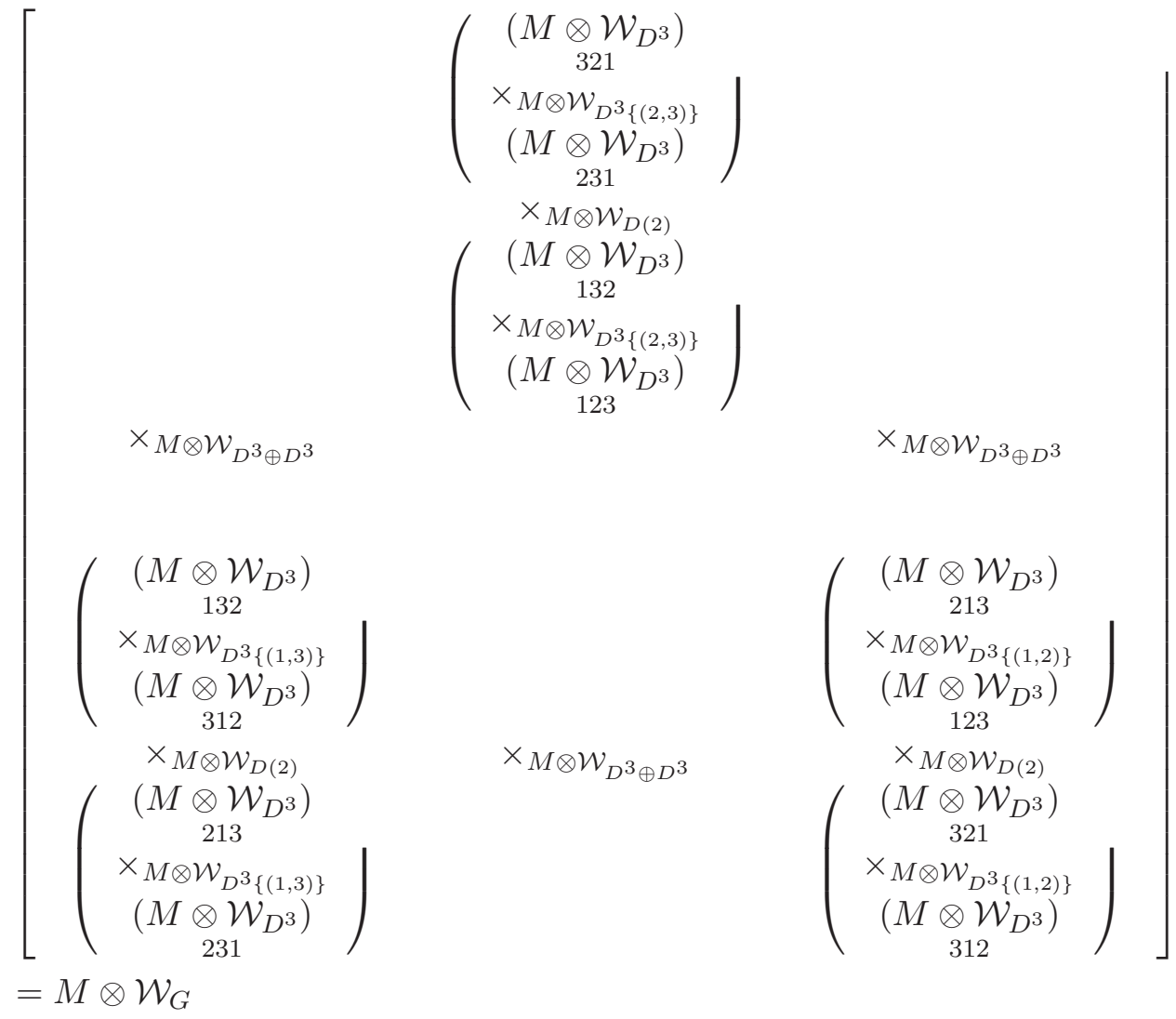

with the diagrams

$$
\begin{aligned}
& \left(\begin{array}{c}
\left(M \otimes \mathcal{W}_{D^{3}}\right) \\
132 \\
\times M \otimes \mathcal{W}_{D^{3}\{(1,3)\}} \\
\left(M \otimes \mathcal{W}_{D^{3}}\right) \\
312
\end{array}\right) \\
& \triangle \quad \rightarrow \quad \times_{M \otimes \mathcal{W}_{D(2)}} \\
& \left(\begin{array}{c}
\left(M \otimes \mathcal{W}_{D^{3}}\right) \\
213 \\
\times M \otimes \mathcal{W}_{D^{3}\{(1,3)\}} \\
\left(M \otimes \mathcal{W}_{D^{3}}\right) \\
231
\end{array}\right) \\
& M \otimes \mathcal{W}_{G} \quad \rightarrow \quad M \otimes \mathcal{W}_{E[2]} \\
& \operatorname{id}_{M} \otimes \mathcal{W}_{k_{2}}
\end{aligned}
$$


and

$$
\begin{aligned}
& \left(\begin{array}{c}
\left(M \otimes \mathcal{W}_{D^{3}}\right) \\
213 \\
\times M \otimes \mathcal{W}_{D^{3}\{(1,2)\}} \\
\left(M \otimes \mathcal{W}_{D^{3}}\right) \\
123
\end{array}\right) \\
& \rightarrow \quad \times_{M \otimes \mathcal{W}_{D(2)}} \\
& \left(\begin{array}{c}
\left(M \otimes \mathcal{W}_{D^{3}}\right) \\
321 \\
\times_{M} \otimes \mathcal{W}_{D^{3}\{(1,2)\}} \\
\left(M \otimes \mathcal{W}_{D^{3}}\right) \\
312
\end{array}\right) \\
& \| \\
& M \otimes \mathcal{W}_{G} \quad \underset{\operatorname{id}_{M} \otimes \mathcal{W}_{k_{3}}}{\rightarrow} \quad M \otimes \mathcal{W}_{E[3]}
\end{aligned}
$$

being commutative, where $\triangle$ in the above diagram is

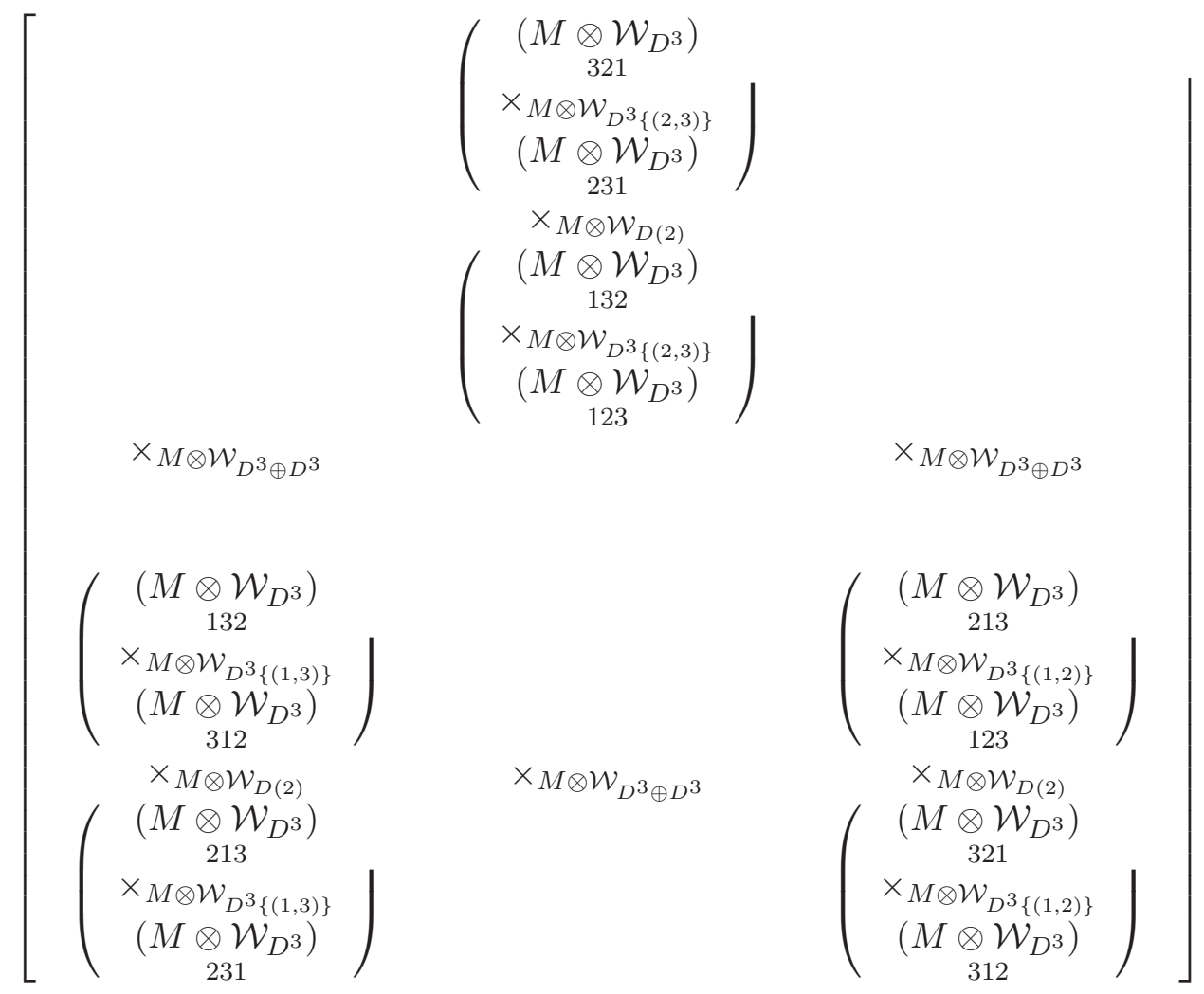

and unnamed arrows are the canonical projections.

The proof of the above theorem follows directly from the following lemma. 
Lemma 32. The following diagram is a limit diagram of Weil algebras:

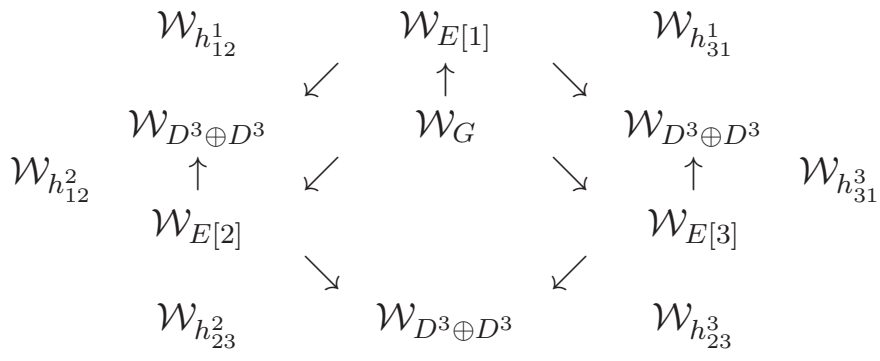

Proof. Let $\gamma_{1} \in \mathcal{W}_{E[1]}, \gamma_{2} \in \mathcal{W}_{E[2]}, \gamma_{3} \in \mathcal{W}_{E[3]}$ and $\gamma \in \mathcal{W}_{G}$ so that they are polynomials with coefficients in $k$ of the following forms:

$$
\begin{aligned}
& \gamma_{1}\left(X_{1}, X_{2}, X_{3}, X_{4}, X_{5}, X_{6}, X_{7}\right) \\
& =a^{1}+a_{1}^{1} X_{1}+a_{2}^{1} X_{2}+a_{3}^{1} X_{3}+a_{4}^{1} X_{4}+a_{5}^{1} X_{5}+a_{6}^{1} X_{6}+a_{7}^{1} X_{7}+a_{12}^{1} X_{1} X_{2}+a_{13}^{1} X_{1} X_{3}+ \\
& a_{14}^{1} X_{1} X_{4}+a_{15}^{1} X_{1} X_{5}+a_{16}^{1} X_{1} X_{6}+a_{23}^{1} X_{2} X_{3}+a_{45}^{1} X_{4} X_{5}+a_{123}^{1} X_{1} X_{2} X_{3}+a_{145}^{1} X_{1} X_{4} X_{5} \\
& \gamma_{2}\left(X_{1}, X_{2}, X_{3}, X_{4}, X_{5}, X_{6}, X_{7}\right) \\
& =a^{2}+a_{1}^{2} X_{1}+a_{2}^{2} X_{2}+a_{3}^{2} X_{3}+a_{4}^{2} X_{4}+a_{5}^{2} X_{5}+a_{6}^{2} X_{6}+a_{7}^{2} X_{7}+a_{12}^{2} X_{1} X_{2}+a_{13}^{2} X_{1} X_{3}+ \\
& a_{23}^{2} X_{2} X_{3}+a_{24}^{2} X_{2} X_{4}+a_{25}^{2} X_{2} X_{5}+a_{26}^{2} X_{2} X_{6}+a_{45}^{2} X_{4} X_{5}+a_{123}^{2} X_{1} X_{2} X_{3}+a_{245}^{2} X_{2} X_{4} X_{5} \\
& \gamma_{3}\left(X_{1}, X_{2}, X_{3}, X_{4}, X_{5}, X_{6}, X_{7}\right) \\
& =a^{3}+a_{1}^{3} X_{1}+a_{2}^{3} X_{2}+a_{3}^{3} X_{3}+a_{4}^{3} X_{4}+a_{5}^{3} X_{5}+a_{6}^{3} X_{6}+a_{7}^{3} X_{7}+a_{12}^{3} X_{1} X_{2}+a_{13}^{3} X_{1} X_{3}+ \\
& a_{23}^{3} X_{2} X_{3}+a_{34}^{3} X_{3} X_{4}+a_{35}^{3} X_{3} X_{5}+a_{36}^{3} X_{3} X_{6}+a_{45}^{3} X_{4} X_{5}+a_{123}^{3} X_{1} X_{2} X_{3}+a_{345}^{3} X_{3} X_{4} X_{5} \\
& \quad \gamma\left(X_{1}, X_{2}, X_{3}, X_{4}, X_{5}, X_{6}, X_{7}, X_{8}\right) \\
& \quad=b+b_{1} X_{1}+b_{2} X_{2}+b_{3} X_{3}+b_{4} X_{4}+b_{5} X_{5}+b_{6} X_{6}+b_{7} X_{7}+b_{8} X_{8}+b_{12} X_{1} X_{2} \\
& \quad+b_{13} X_{1} X_{3}+b_{14} X_{1} X_{4}+b_{23} X_{2} X_{3}+b_{25} X_{2} X_{5}+b_{36} X_{3} X_{6}
\end{aligned}
$$

It is easy to see that

$$
\begin{aligned}
& \mathcal{W}_{h_{12}^{1}}\left(\gamma_{1}\right)\left(X_{1}, X_{2}, X_{3}, X_{4}, X_{5}, X_{6}\right) \\
& =a^{1}+a_{1}^{1} X_{1}+a_{2}^{1} X_{2}+a_{3}^{1} X_{3}+a_{6}^{1} X_{2} X_{3}+a_{12}^{1} X_{1} X_{2}+a_{13}^{1} X_{1} X_{3}+a_{16}^{1} X_{1} X_{2} X_{3} \\
& +a_{23}^{1} X_{2} X_{3}+a_{123}^{1} X_{1} X_{2} X_{3}+a_{1}^{1} X_{4}+a_{4}^{1} X_{5}+a_{5}^{1} X_{6}+a_{14}^{1} X_{4} X_{5}+a_{15}^{1} X_{4} X_{6}+a_{45}^{1} X_{5} X_{6} \\
& +a_{145}^{1} X_{4} X_{5} X_{6} \\
& =a^{1}+a_{1}^{1} X_{1}+a_{2}^{1} X_{2}+a_{3}^{1} X_{3}+a_{12}^{1} X_{1} X_{2}+a_{13}^{1} X_{1} X_{3}+\left(a_{6}^{1}+a_{23}^{1}\right) X_{2} X_{3} \\
& +\left(a_{16}^{1}+a_{123}^{1}\right) X_{1} X_{2} X_{3}+a_{1}^{1} X_{4}+a_{4}^{1} X_{5}+a_{5}^{1} X_{6}+a_{14}^{1} X_{4} X_{5}+a_{15}^{1} X_{4} X_{6}+a_{45}^{1} X_{5} X_{6} \\
& +a_{145}^{1} X_{4} X_{5} X_{6}
\end{aligned}
$$




$$
\begin{aligned}
& \mathcal{W}_{h_{12}^{2}}\left(\gamma_{2}\right)\left(X_{1}, X_{2}, X_{3}, X_{4}, X_{5}, X_{6}\right) \\
& =a^{2}+a_{2}^{2} X_{2}+a_{4}^{2} X_{3}+a_{5}^{2} X_{1}+a_{6}^{2} X_{1} X_{3}+a_{7}^{2} X_{1} X_{2} X_{3}+a_{24}^{2} X_{2} X_{3}+a_{25}^{2} X_{1} X_{2} \\
& +a_{26}^{2} X_{1} X_{2} X_{3}+a_{45}^{2} X_{1} X_{3}+a_{245}^{2} X_{1} X_{2} X_{3}+a_{1}^{2} X_{4}+a_{2}^{2} X_{5}+a_{3}^{2} X_{6}+a_{12}^{2} X_{4} X_{5} \\
& +a_{13}^{2} X_{4} X_{6}+a_{23}^{2} X_{5} X_{6}+a_{123}^{2} X_{4} X_{5} X_{6} \\
& =a^{2}+a_{5}^{2} X_{1}+a_{2}^{2} X_{2}+a_{4}^{2} X_{3}+a_{25}^{2} X_{1} X_{2}+\left(a_{6}^{2}+a_{45}^{2}\right) X_{1} X_{3}+a_{24}^{2} X_{2} X_{3}+ \\
& \left(a_{7}^{2}+a_{26}^{2}+a_{245}^{2}\right) X_{1} X_{2} X_{3}+a_{1}^{2} X_{4}+a_{2}^{2} X_{5}+a_{3}^{2} X_{6}+a_{12}^{2} X_{4} X_{5}+a_{13}^{2} X_{4} X_{6}+a_{23}^{2} X_{5} X_{6} \\
& +a_{123}^{2} X_{4} X_{5} X_{6}
\end{aligned}
$$

Therefore the condition that $\mathcal{W}_{h_{12}^{1}}\left(\gamma_{1}\right)=\mathcal{W}_{h_{12}^{2}}\left(\gamma_{2}\right)$ is equivalent to the following conditions as a whole:

$$
\begin{aligned}
a^{1} & =a^{2} \\
a_{1}^{1} & =a_{5}^{2}, a_{2}^{1}=a_{2}^{2}, a_{3}^{1}=a_{4}^{2}, a_{1}^{1}=a_{1}^{2}, a_{4}^{1}=a_{2}^{2}, a_{5}^{1}=a_{3}^{2} \\
a_{12}^{1} & =a_{25}^{2}, a_{13}^{1}=a_{6}^{2}+a_{45}^{2}, a_{6}^{1}+a_{23}^{1}=a_{24}^{2}, a_{14}^{1}=a_{12}^{2}, a_{15}^{1}=a_{13}^{2}, \\
a_{45}^{1} & =a_{23}^{2} \\
a_{16}^{1}+a_{123}^{1} & =a_{7}^{2}+a_{26}^{2}+a_{245}^{2}, a_{145}^{1}=a_{123}^{2}
\end{aligned}
$$

By the same token, the condition that $\mathcal{W}_{h_{23}^{2}}\left(\gamma_{2}\right)=\mathcal{W}_{h_{23}^{3}}\left(\gamma_{3}\right)$ is equivalent to the following conditions as a whole:

$$
\begin{aligned}
a^{2} & =a^{3} \\
a_{2}^{2} & =a_{5}^{3}, a_{3}^{2}=a_{3}^{3}, a_{1}^{2}=a_{4}^{3}, a_{2}^{2}=a_{2}^{3}, a_{4}^{2}=a_{3}^{3}, a_{5}^{2}=a_{1}^{3} \\
a_{23}^{2} & =a_{35}^{3}, a_{12}^{2}=a_{6}^{3}+a_{45}^{3}, a_{6}^{2}+a_{13}^{2}=a_{34}^{3}, a_{24}^{2}=a_{23}^{3}, a_{25}^{2}=a_{12}^{3}, \\
a_{45}^{2} & =a_{13}^{3} \\
a_{26}^{2}+a_{123}^{2} & =a_{7}^{3}+a_{36}^{3}+a_{345}^{3}, a_{245}^{2}=a_{123}^{3}
\end{aligned}
$$

By the same token again, the condition that $\mathcal{W}_{h_{31}^{3}}\left(\gamma_{3}\right)=\mathcal{W}_{h_{31}^{1}}\left(\gamma_{1}\right)$ is equivalent to the following conditions as a whole:

$$
\begin{aligned}
a^{3} & =a^{1} \\
a_{3}^{3} & =a_{5}^{1}, a_{1}^{3}=a_{1}^{1}, a_{2}^{3}=a_{4}^{1}, a_{3}^{3}=a_{3}^{1}, a_{4}^{3}=a_{1}^{1}, a_{5}^{3}=a_{2}^{1} \\
a_{13}^{3} & =a_{15}^{1}, a_{23}^{3}=a_{6}^{1}+a_{45}^{1}, a_{6}^{3}+a_{12}^{3}=a_{14}^{1}, a_{34}^{3}=a_{13}^{1}, a_{35}^{3}=a_{23}^{1}, \\
a_{45}^{3} & =a_{12}^{1} \\
a_{36}^{3}+a_{123}^{3} & =a_{7}^{1}+a_{16}^{1}+a_{145}^{1}, a_{345}^{3}=a_{123}^{1}
\end{aligned}
$$

The three conditions (1), (5) and (9) can be combined into

$$
a^{1}=a^{2}=a^{3}
$$


The three conditions (2), (6) and (10) are to be superseded by the following three conditions as a whole:

$$
\begin{aligned}
& a_{1}^{1}=a_{1}^{2}=a_{1}^{3}=a_{5}^{2}=a_{4}^{3} \\
& a_{2}^{1}=a_{2}^{2}=a_{2}^{3}=a_{4}^{1}=a_{5}^{3} \\
& a_{3}^{1}=a_{3}^{2}=a_{3}^{3}=a_{5}^{1}=a_{4}^{2}
\end{aligned}
$$

The three conditions (3), (7) and (11) are equivalent to the following six conditions as a whole:

$$
\begin{aligned}
& a_{12}^{1}=a_{12}^{2}=a_{12}^{3} \\
& a_{13}^{1}=a_{13}^{2}=a_{13}^{3} \\
& a_{23}^{1}=a_{23}^{2}=a_{23}^{3} \\
& a_{14}^{1}=a_{12}^{1}+a_{6}^{3}, a_{15}^{1}=a_{13}^{1}-a_{6}^{2}, a_{45}^{1}=a_{23}^{1} \\
& a_{24}^{2}=a_{23}^{2}+a_{6}^{1}, a_{25}^{2}=a_{12}^{2}-a_{6}^{3}, a_{45}^{2}=a_{13}^{2} \\
& a_{34}^{3}=a_{13}^{3}+a_{6}^{2}, a_{35}^{3}=a_{23}^{3}-a_{6}^{1}, a_{45}^{3}=a_{12}^{3}
\end{aligned}
$$

The conditions (4), (8) and (12) imply that

$$
\begin{aligned}
a_{7}^{1} & +a_{7}^{2}+a_{7}^{3} \\
= & \left(a_{36}^{3}+a_{123}^{3}-a_{16}^{1}-a_{145}^{1}\right)+\left(a_{16}^{1}+a_{123}^{1}-a_{26}^{2}-a_{245}^{2}\right) \\
& +\left(a_{26}^{2}+a_{123}^{2}-a_{36}^{3}-a_{345}^{3}\right) \\
= & \left(a_{36}^{3}+a_{123}^{3}-a_{16}^{1}-a_{123}^{2}\right)+\left(a_{16}^{1}+a_{123}^{1}-a_{26}^{2}-a_{123}^{3}\right) \\
& +\left(a_{26}^{2}+a_{123}^{2}-a_{36}^{3}-a_{123}^{1}\right) \\
= & 0
\end{aligned}
$$

Therefore the three conditions (4), (8) and (12) are to be replaced by the following five conditions as a whole:

$$
\begin{aligned}
a_{145}^{1}-a_{123}^{1} & =a_{7}^{3}+a_{36}^{3}-a_{26}^{2} \\
a_{245}^{2}-a_{123}^{2} & =a_{7}^{1}+a_{16}^{1}-a_{36}^{3} \\
a_{345}^{3}-a_{123}^{3} & =a_{7}^{2}+a_{26}^{2}-a_{16}^{1} \\
a_{145}^{1} & =a_{123}^{2}, a_{245}^{2}=a_{123}^{3} \\
a_{7}^{1}+a_{7}^{2}+a_{7}^{3} & =0
\end{aligned}
$$

Indeed, the condition that $a_{345}^{3}=a_{123}^{1}$ is derivable from the above five conditions, as is to be demonstrated in the following:

$$
a_{345}^{3}
$$




$$
\begin{aligned}
& =a_{123}^{3}+a_{7}^{2}+a_{26}^{2}-a_{16}^{1} \quad[(26)] \\
& =a_{245}^{2}+a_{7}^{2}+a_{26}^{2}-a_{16}^{1} \quad[(27)] \\
& =a_{123}^{2}+a_{7}^{1}-a_{36}^{3}+a_{7}^{2}+a_{26}^{2} \\
& =a_{145}^{1}+a_{7}^{1}-a_{36}^{3}+a_{7}^{2}+a_{26}^{2} \\
& =a_{123}^{1}+a_{7}^{1}+a_{7}^{2}+a_{7}^{3} \quad[(24)] \\
& =a_{123}^{1}
\end{aligned}
$$

Now it is not difficult to see that $\mathcal{W}_{h_{12}^{1}}\left(\gamma_{1}\right)=\mathcal{W}_{h_{12}^{2}}\left(\gamma_{2}\right), \mathcal{W}_{h_{23}^{2}}\left(\gamma_{2}\right)=\mathcal{W}_{h_{23}^{3}}\left(\gamma_{3}\right)$ and $\mathcal{W}_{h_{31}^{3}}\left(\gamma_{3}\right)=\mathcal{W}_{h_{31}^{1}}\left(\gamma_{1}\right)$ exactly when there exists $\gamma \in \mathcal{W}_{G}$ with $\gamma_{i}=\mathcal{W}_{k_{i}}(\gamma)$ $(i=1,2,3)$, in which $\gamma$ should uniquely be of the following form:

$$
\begin{aligned}
& \gamma\left(X_{1}, X_{2}, X_{3}, X_{4}, X_{5}, X_{6}, X_{7}, X_{8}\right) \\
& =a^{1}+a_{1}^{1} X_{1}+a_{2}^{1} X_{2}+a_{3}^{1} X_{3}+a_{6}^{1} X_{4}+a_{6}^{2} X_{5}+a_{6}^{3} X_{6}+a_{7}^{1} X_{7}+a_{7}^{2} X_{8}+a_{12}^{1} X_{1} X_{2} \\
& +a_{13}^{1} X_{1} X_{3}+a_{16}^{1} X_{1} X_{4}+\left(a_{23}^{2}+a_{6}^{1}\right) X_{2} X_{3}+a_{26}^{2} X_{2} X_{5}+a_{36}^{3} X_{3} X_{6}
\end{aligned}
$$

This completes the proof of the theorem.

Notation 33. We will introduce three notations.

1. We will write

$$
\begin{aligned}
& \zeta^{\left(*_{123}-*_{132}\right)-\left(*_{231}-*_{321}\right)} \text { : }
\end{aligned}
$$

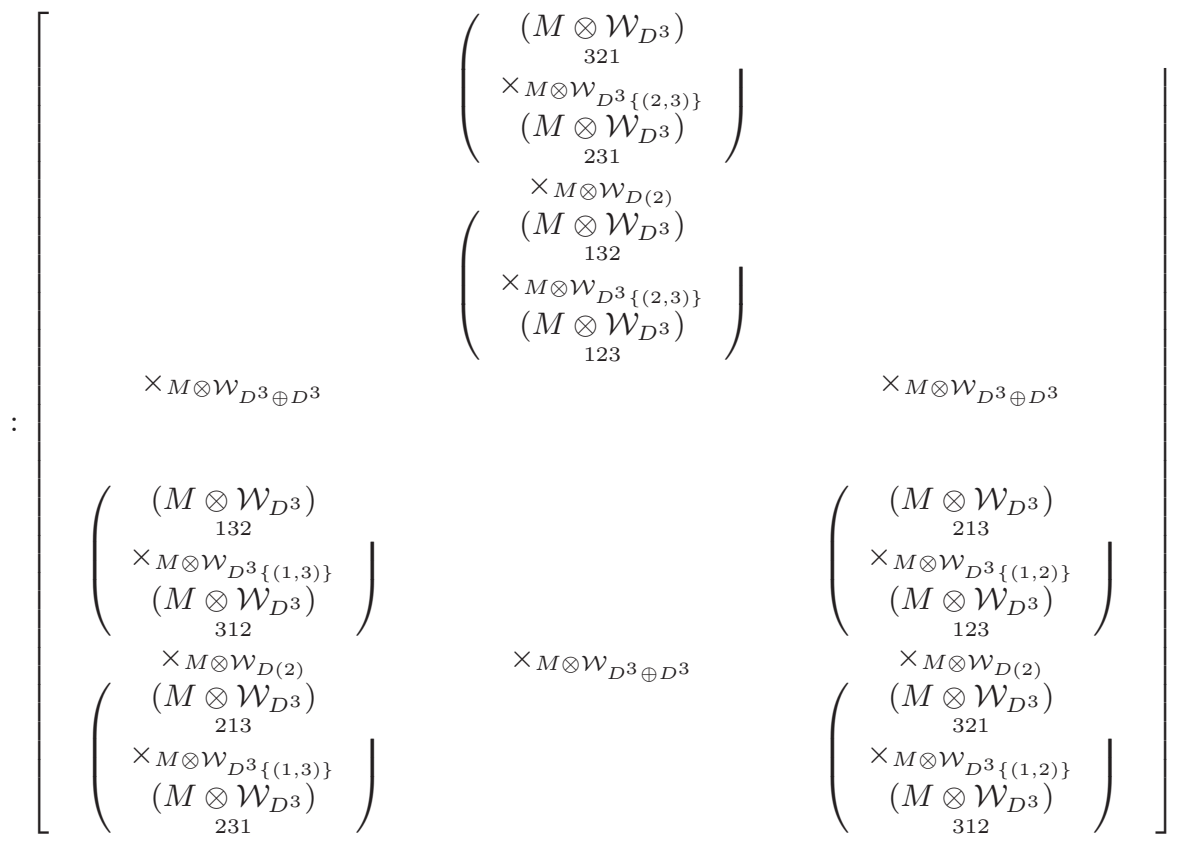


$\rightarrow M \otimes \mathcal{W}_{D}$

for the composition of morphisms

$$
\begin{aligned}
& \pi^{\triangle}\left(\left(\left(M \otimes \mathcal{W}_{D^{3}}\right) \times_{M \otimes} \otimes \mathcal{W}_{D^{3}\{(2,3)\}}\left(\underset{231}{M \otimes \mathcal{W}_{D^{3}}}\right)\right) \times_{M \otimes \mathcal{W}_{D(2)}}\left(\underset{132}{\left.\left.\left(M \otimes \mathcal{W}_{D^{3}}\right) \times_{M \otimes \mathcal{W}_{D^{3}\{(2,3)\}}}\left(M \otimes \mathcal{W}_{D^{3}}\right)\right)\right)}:\right.\right. \\
& {\left[\begin{array}{c}
\left(M \otimes \mathcal{W}_{D^{3}}\right) \\
321 \\
\times M \otimes \mathcal{W}_{D^{3}\{(2,3)\}} \\
\left(M \otimes \mathcal{W}_{D^{3}}\right) \\
231 \\
\times_{M \otimes \mathcal{W}_{D(2)}} \\
\left(M \otimes \mathcal{W}_{D^{3}}\right) \\
132 \\
\times_{M \otimes \mathcal{W}_{D^{3}}\{(2,3)\}} \\
\left(M \otimes \mathcal{W}_{D^{3}}\right) \\
123
\end{array}\right)} \\
& \times_{M \otimes \mathcal{W}_{D^{3} \oplus D^{3}}} \quad \times_{M \otimes \mathcal{W}_{D^{3} \oplus D^{3}}}
\end{aligned}
$$

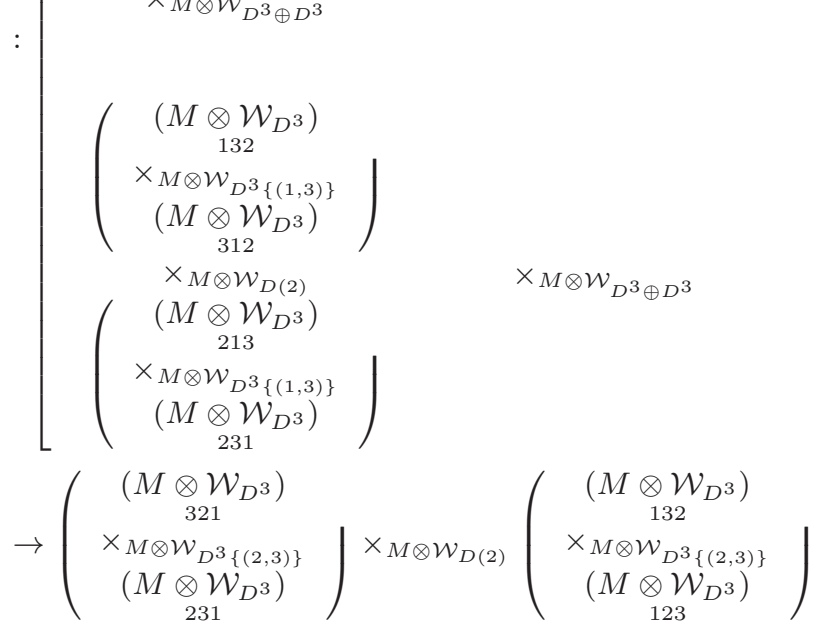

$$
\begin{aligned}
& \zeta^{-} \times_{M \otimes \mathcal{W}_{D(2)}} \zeta^{-}: \\
& :\left(\begin{array}{c}
\left(M \otimes \mathcal{W}_{D^{3}}\right) \\
321 \\
\times_{M \otimes \mathcal{W}_{D^{3}\{(2,3)\}}} \\
\left(M \otimes \mathcal{W}_{D^{3}}\right) \\
231
\end{array}\right) \times_{M \otimes \mathcal{W}_{D(2)}}\left(\begin{array}{c}
\left(M \otimes \mathcal{W}_{D^{3}}\right) \\
132 \\
\times_{M \otimes \mathcal{W}_{D^{3}\{(2,3)\}}} \\
\left(M \otimes \mathcal{W}_{D^{3}}\right) \\
123
\end{array}\right) \\
& \rightarrow\left(M \otimes \mathcal{W}_{D^{2}}\right) \times_{M \otimes \mathcal{W}_{D(2)}}\left(M \otimes \mathcal{W}_{D^{2}}\right) \\
& \zeta^{-}:\left(M \otimes \mathcal{W}_{D^{2}}\right) \times_{M \otimes \mathcal{W}_{D(2)}}\left(M \otimes \mathcal{W}_{D^{2}}\right) \rightarrow M \otimes \mathcal{W}_{D}
\end{aligned}
$$

in succession. 
2. We will write the morphism

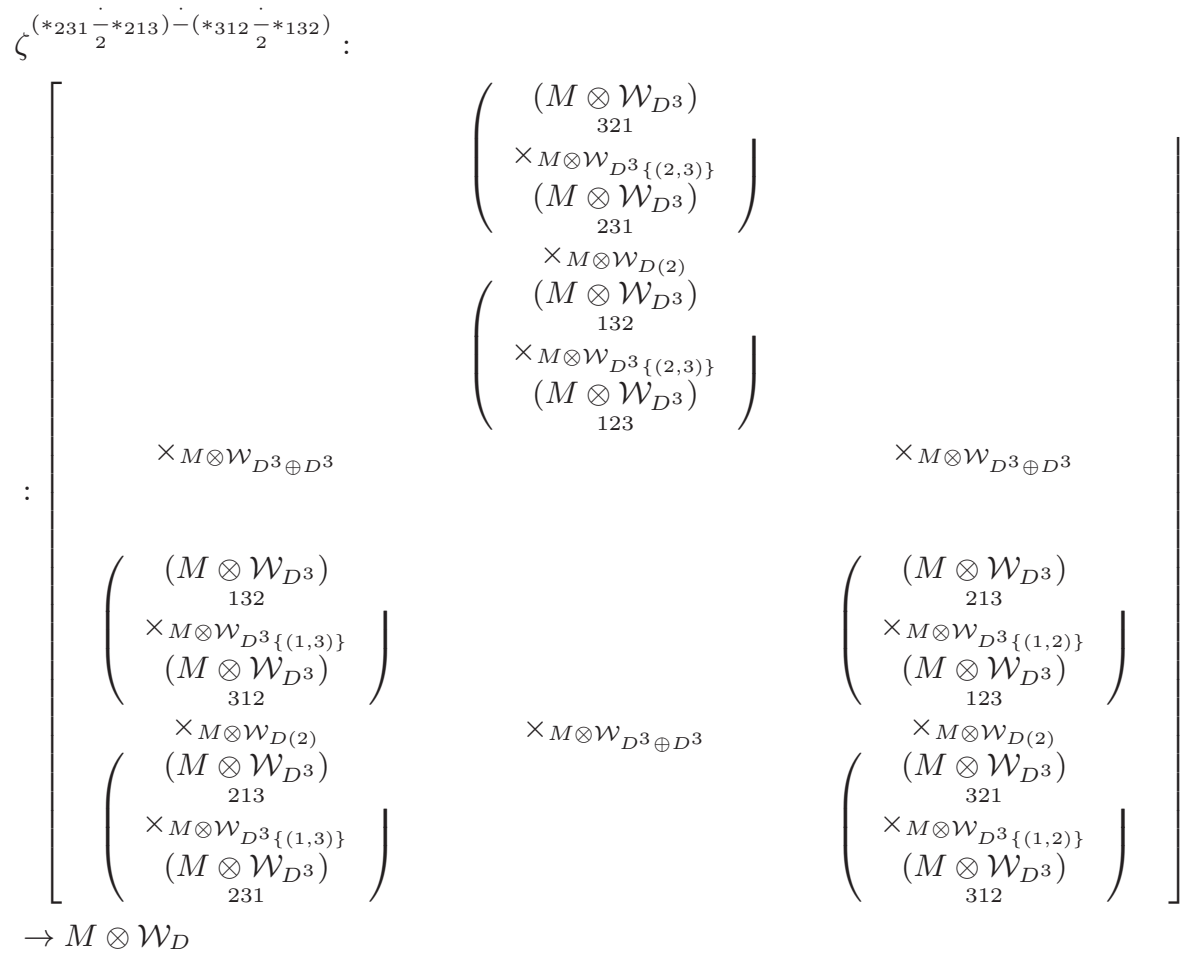

for the composition of morphisms 


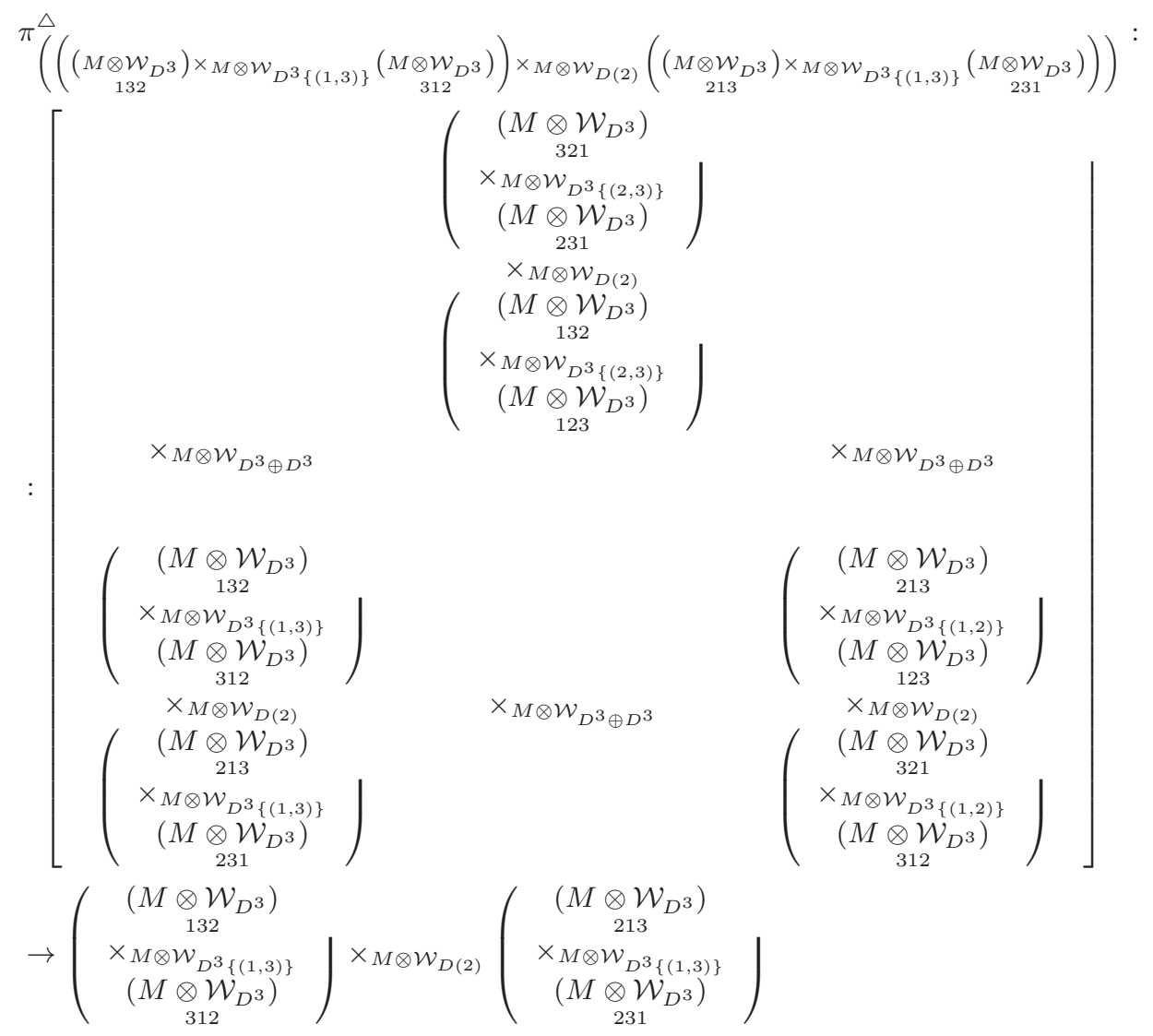

$$
\begin{aligned}
& \zeta^{\dot{\overline{2}}} \times_{M \otimes \mathcal{W}_{D(2)}} \zeta^{\dot{\overline{2}}}: \\
& \quad\left(\begin{array}{c}
\left(M \otimes \mathcal{W}_{D^{3}}\right) \\
132 \\
\times_{M \otimes \mathcal{W}_{D^{3}\{(1,3)\}}} \\
\left(M \otimes \mathcal{W}_{D^{3}}\right) \\
312
\end{array}\right) \times_{M \otimes \mathcal{W}_{D(2)}}\left(\begin{array}{c}
\left(M \otimes \mathcal{W}_{D^{3}}\right) \\
{ }_{213} \\
\times_{M \otimes \mathcal{W}_{D^{3}}\{(1,3)\}} \\
\left(M \otimes \mathcal{W}_{D^{3}}\right) \\
{ }_{231}
\end{array}\right) \\
& \rightarrow\left(M \otimes \mathcal{W}_{D^{2}}\right) \times_{M \otimes \mathcal{W}_{D(2)}}\left(M \otimes \mathcal{W}_{D^{2}}\right) \\
& \quad \zeta^{-}:\left(M \otimes \mathcal{W}_{D^{2}}\right) \times_{M \otimes \mathcal{W}_{D(2)}}\left(M \otimes \mathcal{W}_{D^{2}}\right) \rightarrow M \otimes \mathcal{W}_{D}
\end{aligned}
$$

in succession.

3. We will write the morphism

$$
\zeta^{\left(*_{312} \frac{\dot{-} *}{3} *_{321}\right)-\left(*_{123} \frac{\dot{*} * 213)}{3}\right.}:
$$




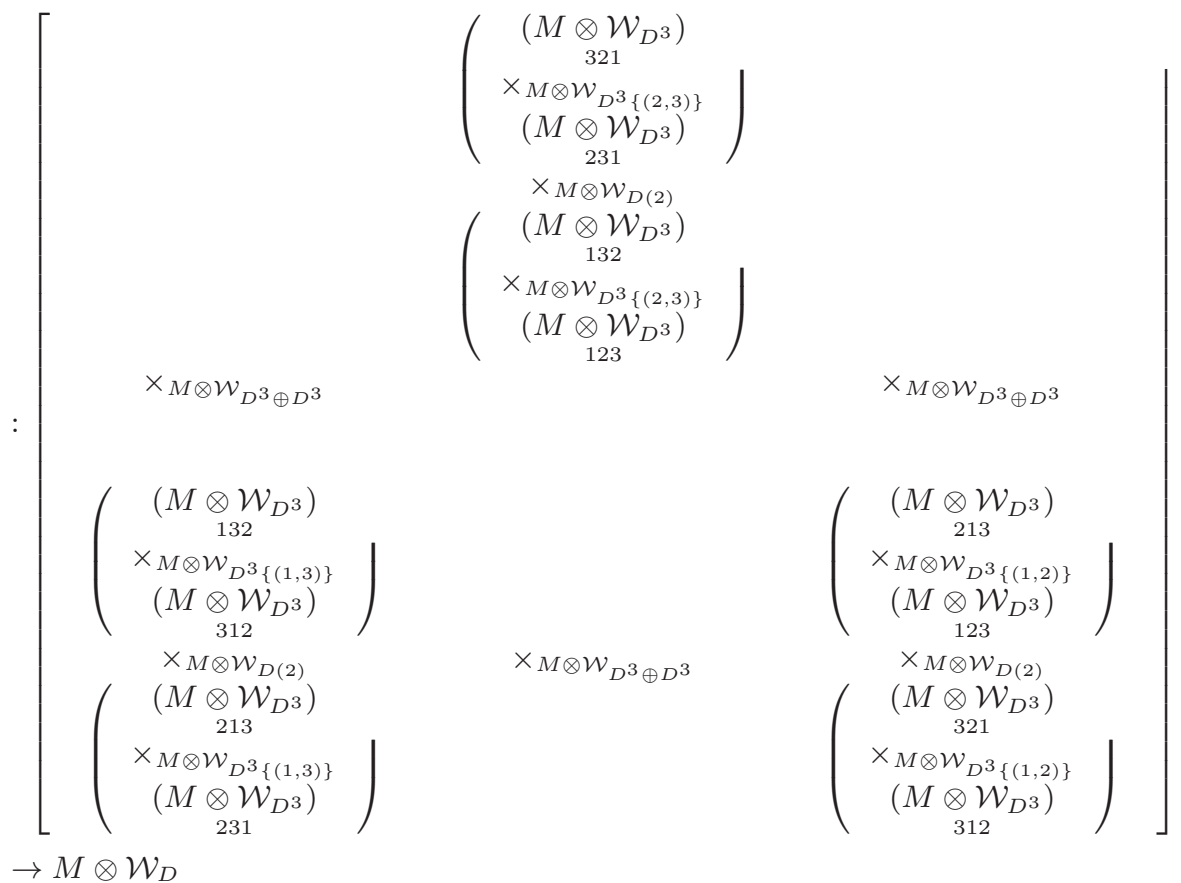

for the composition of morphisms 


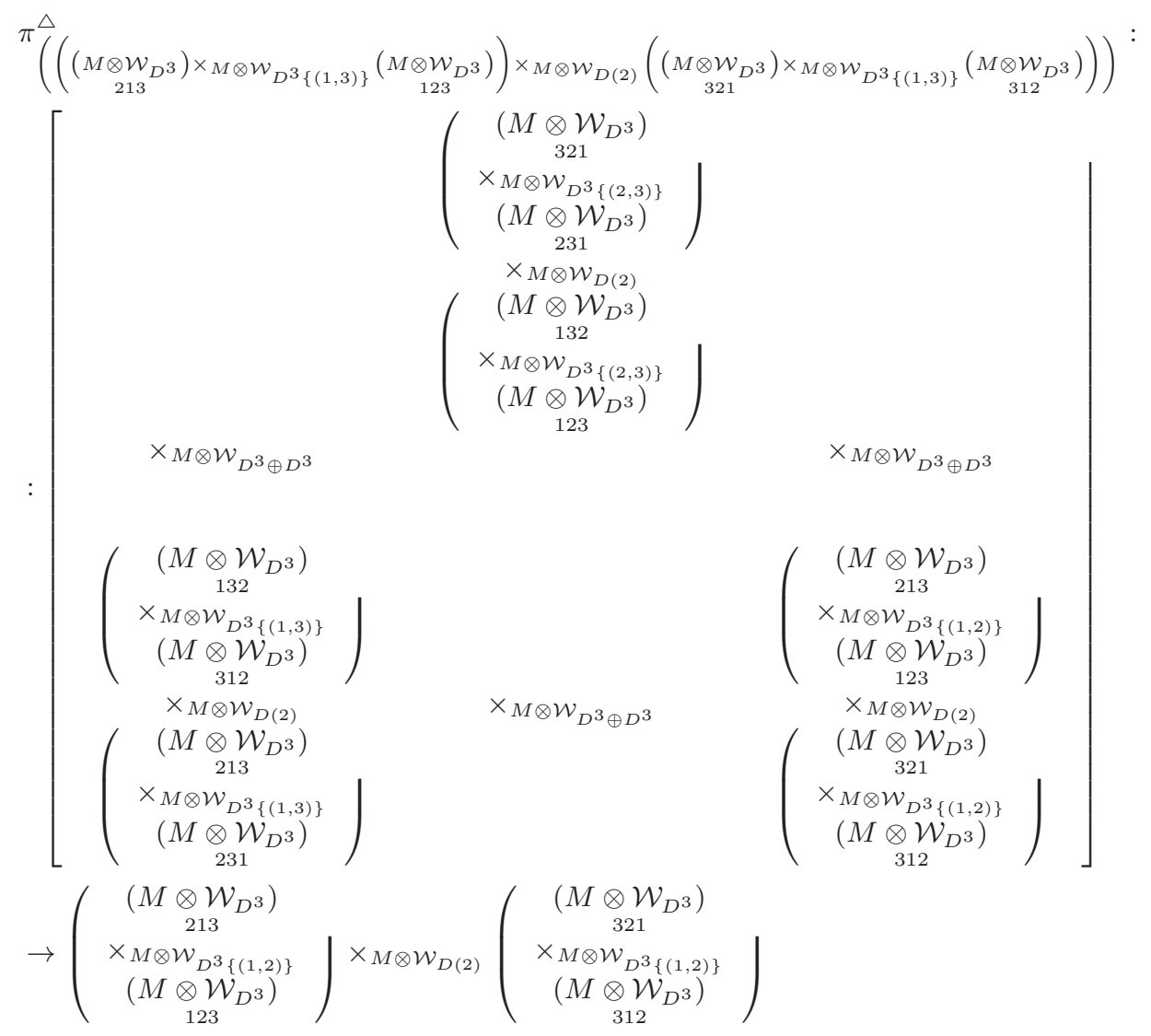

$$
\begin{aligned}
& \zeta^{\dot{\overline{3}}} \times_{M \otimes \mathcal{W}_{D(2)}} \zeta^{\dot{\overline{3}}} \\
& :\left(\begin{array}{c}
\left(M \otimes \mathcal{W}_{D^{3}}\right) \\
213 \\
\times_{M \otimes \mathcal{W}_{D^{3}\{(1,2)\}}} \\
\left(M \otimes \mathcal{W}_{D^{3}}\right) \\
123
\end{array}\right) \times_{M \otimes \mathcal{W}_{D(2)}}\left(\begin{array}{c}
\left(M \otimes \mathcal{W}_{D^{3}}\right) \\
321 \\
\times_{M \otimes \mathcal{W}_{D^{3}\{(1,2)\}}} \\
\left(M \otimes \mathcal{W}_{D^{3}}\right) \\
312
\end{array}\right) \\
& \rightarrow\left(M \otimes \mathcal{W}_{D^{2}}\right) \times_{M \otimes \mathcal{W}_{D(2)}}\left(M \otimes \mathcal{W}_{D^{2}}\right) \\
& \quad \dot{\zeta^{-}}:\left(M \otimes \mathcal{W}_{D^{2}}\right) \times_{M \otimes \mathcal{W}_{D(2)}}\left(M \otimes \mathcal{W}_{D^{2}}\right) \rightarrow M \otimes \mathcal{W}_{D}
\end{aligned}
$$

in succession.

Theorem 34. (The General Jacobi Identity) The three morphisms $\zeta^{\left(*_{123}-*_{132}\right)-\left(*_{231}-*_{321}\right)}:$ 


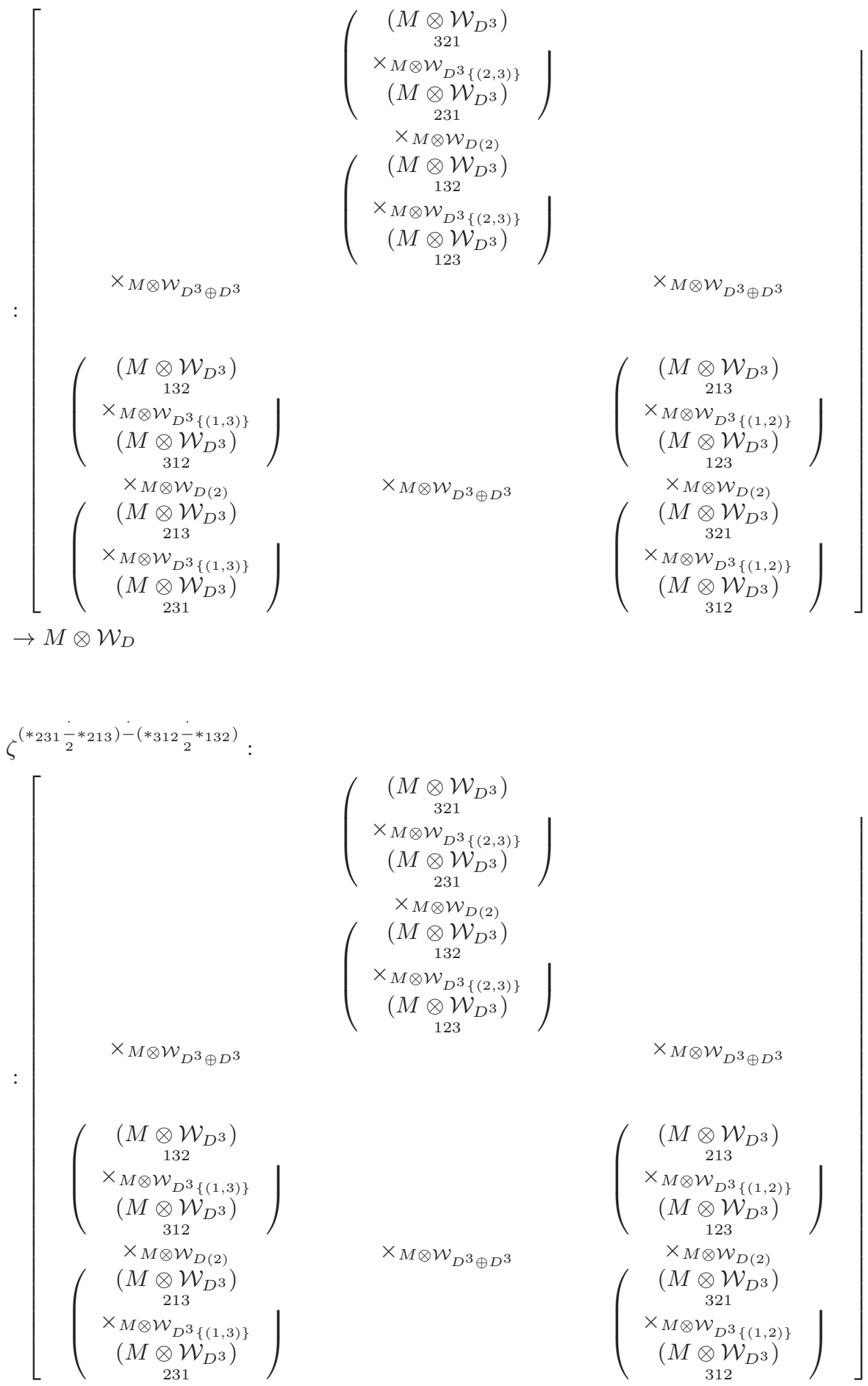


$\rightarrow M \otimes \mathcal{W}_{D}$

$\zeta^{\left(*_{312}-*_{321}\right)-\left(*_{123}-*_{213}\right)}:$

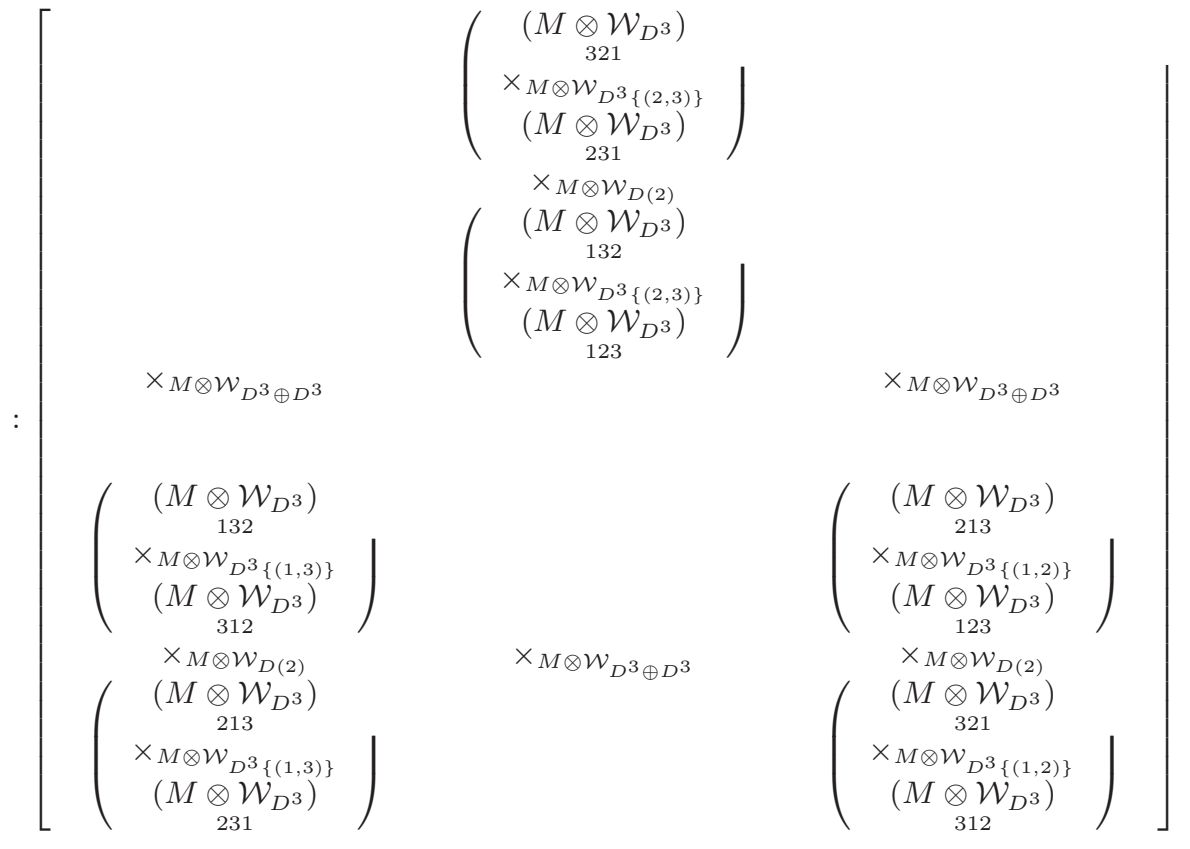

$\rightarrow M \otimes \mathcal{W}_{D}$

sum up only to vanish.

Proof. The proof is divided into four steps.

1. The morphism 


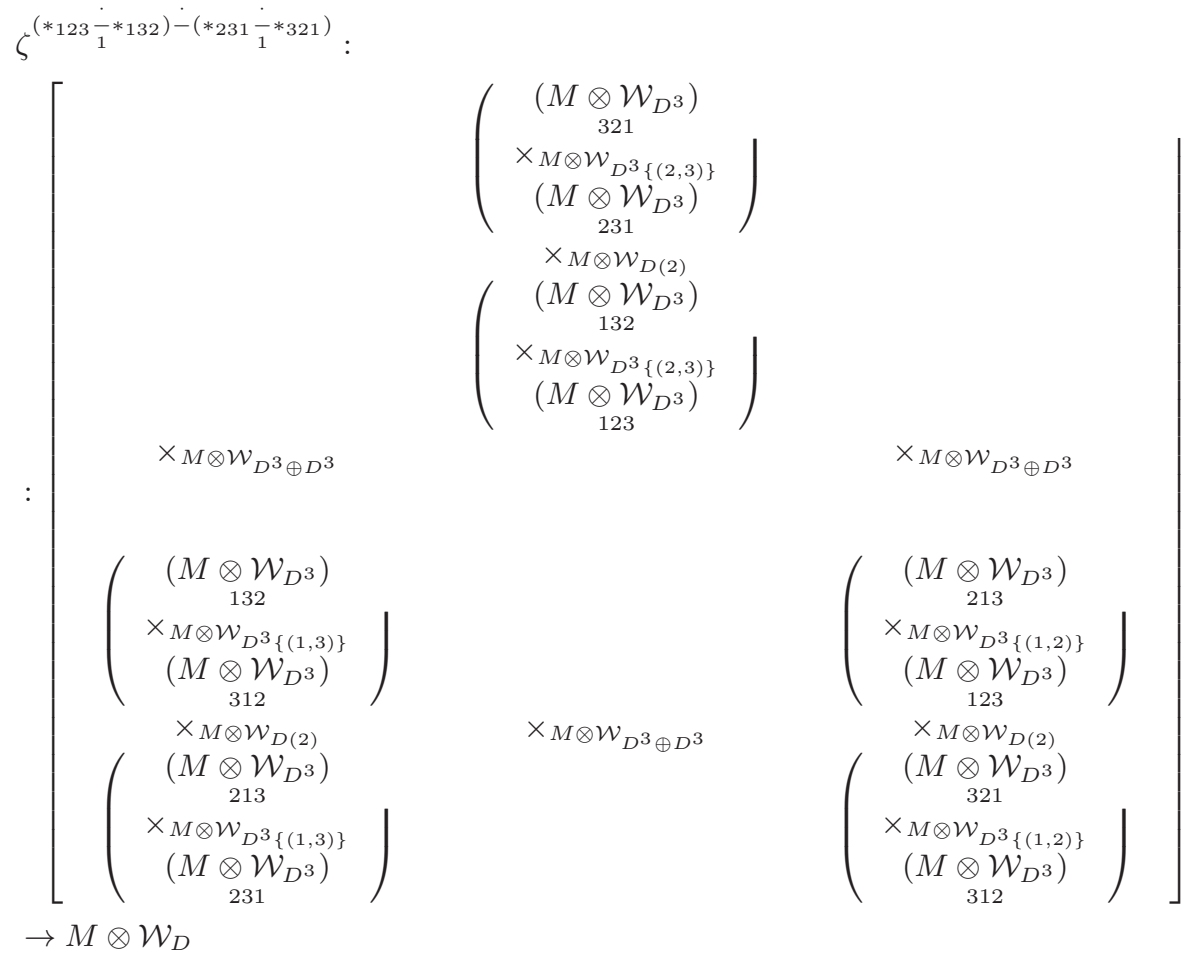

is equivalent to the composition of

$$
\operatorname{id}_{M} \otimes k_{1}: M \otimes \mathcal{W}_{G} \rightarrow M \otimes \mathcal{W}_{E[1]}
$$

$\operatorname{id}_{M} \otimes \mathcal{W}_{\left(d_{1}, d_{2}, d_{3}\right) \in D^{3}\{(1,3),(2,3)\} \mapsto\left(d_{1}, 0,0,0,0, d_{2}, d_{3}\right) \in E[1]}: M \otimes \mathcal{W}_{E[1]} \rightarrow$

$$
M \otimes \mathcal{W}_{D^{3}\{(1,3),(2,3)\}}
$$

$\operatorname{id}_{M} \otimes \mathcal{W}_{d \in D \mapsto(0,0, d) \in D^{3}\{(1,3),(2,3)\}}: M \otimes \mathcal{W}_{D^{3}\{(1,3),(2,3)\}} \rightarrow M \otimes \mathcal{W}_{D}$

in succession, which results in

$$
\operatorname{id}_{M} \otimes \mathcal{W}_{d \in D \mapsto(0,0,0,0,0,0, d, 0) \in G}: M \otimes \mathcal{W}_{G} \rightarrow M \otimes \mathcal{W}_{D}
$$

2. The morphism

$$
\zeta^{\left(* 231 \frac{\dot{-}}{2} *_{213}\right)-\left(* 312 \frac{\dot{*}}{2} *_{132}\right)}:
$$




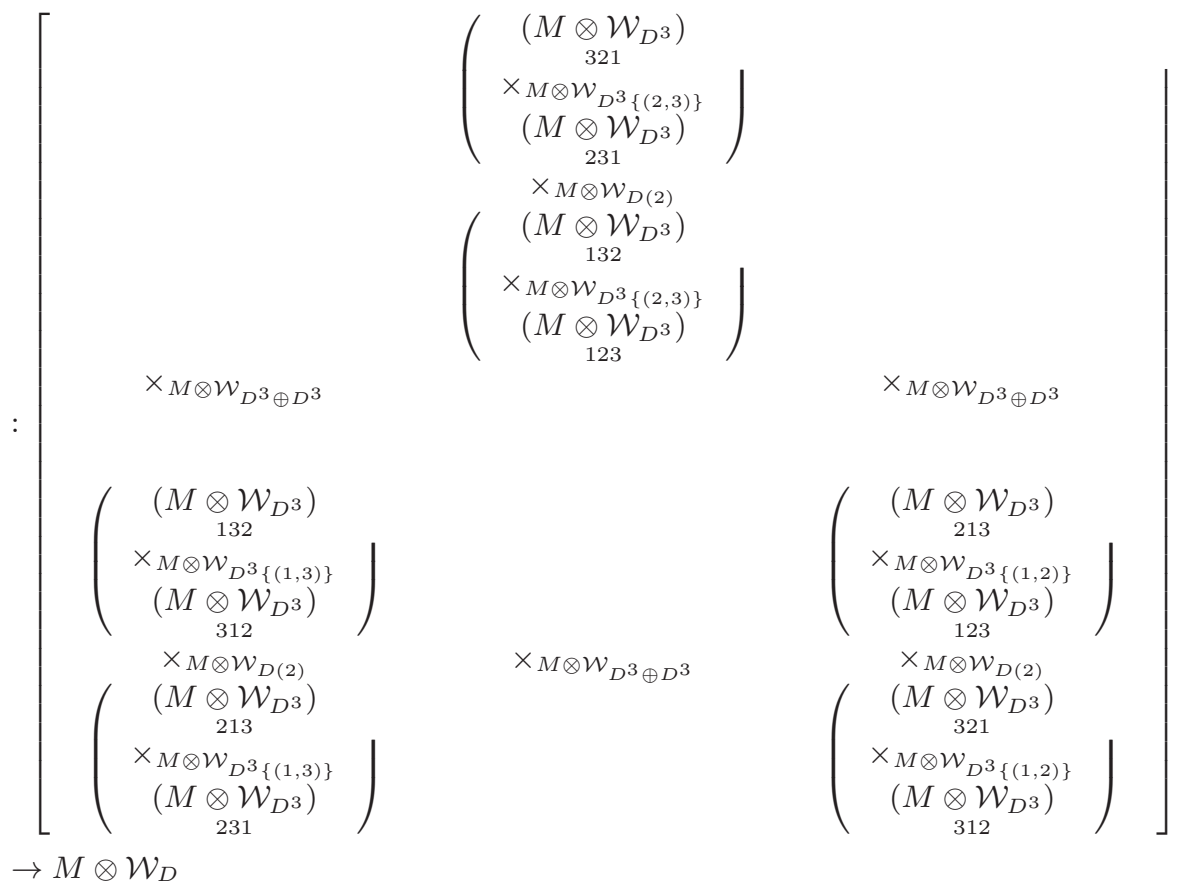

is equivalent to the composition of

$$
\operatorname{id}_{M} \otimes k_{2}: M \otimes \mathcal{W}_{G} \rightarrow M \otimes \mathcal{W}_{E[2]}
$$

$\operatorname{id}_{M} \otimes \mathcal{W}_{\left(d_{1}, d_{2}, d_{3}\right) \in D^{3}\{(1,3),(2,3)\} \mapsto\left(0, d_{1}, 0,0,0, d_{2}, d_{3}\right) \in E[2]}: M \otimes \mathcal{W}_{E[2]} \rightarrow$

$$
M \otimes \mathcal{W}_{D^{3}\{(1,3),(2,3)\}}
$$

$\operatorname{id}_{M} \otimes \mathcal{W}_{d \in D \mapsto(0,0, d) \in D^{3}\{(1,3),(2,3)\}}: M \otimes \mathcal{W}_{D^{3}\{(1,3),(2,3)\}} \rightarrow M \otimes \mathcal{W}_{D}$

in succession, which results in

$$
\operatorname{id}_{M} \otimes \mathcal{W}_{d \in D \mapsto(0,0,0,0,0,0,0, d) \in G}: M \otimes \mathcal{W}_{G} \rightarrow M \otimes \mathcal{W}_{D}
$$

3. The morphism

$$
\zeta^{\left(*_{312} \frac{\dot{-} *}{3} *_{321}\right)-\left(*_{123} \frac{\dot{*} * 213)}{3}\right.}:
$$




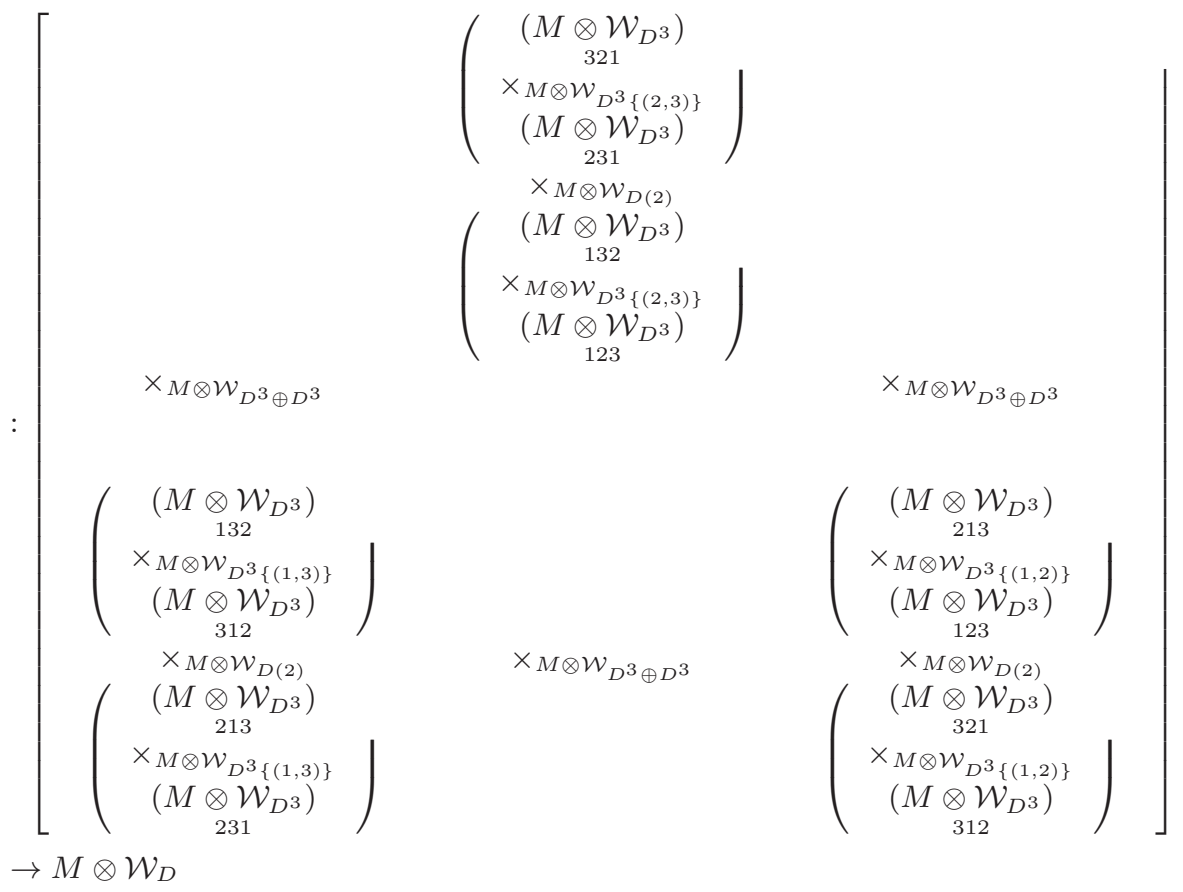

is equivalent to the composition of

$$
\operatorname{id}_{M} \otimes k_{3}: M \otimes \mathcal{W}_{G} \rightarrow M \otimes \mathcal{W}_{E[3]}
$$

$\operatorname{id}_{M} \otimes \mathcal{W}_{\left(d_{1}, d_{2}, d_{3}\right) \in D^{3}\{(1,3),(2,3)\} \mapsto\left(0,0, d_{1}, 0,0, d_{2}, d_{3}\right) \in E[3]}: M \otimes \mathcal{W}_{E[3]}$

$$
\rightarrow M \otimes \mathcal{W}_{D^{3}\{(1,3),(2,3)\}}
$$

$\operatorname{id}_{M} \otimes \mathcal{W}_{d \in D \mapsto(0,0, d) \in D^{3}\{(1,3),(2,3)\}}: M \otimes \mathcal{W}_{D^{3}\{(1,3),(2,3)\}} \rightarrow M \otimes \mathcal{W}_{D}$

in succession, which results in

$$
\operatorname{id}_{M} \otimes \mathcal{W}_{d \in D \mapsto(0,0,0,0,0,0,-d,-d) \in G}: M \otimes \mathcal{W}_{G} \rightarrow M \otimes \mathcal{W}_{D}
$$

\section{Therefore}

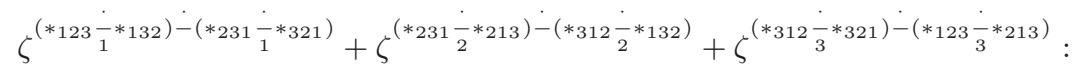




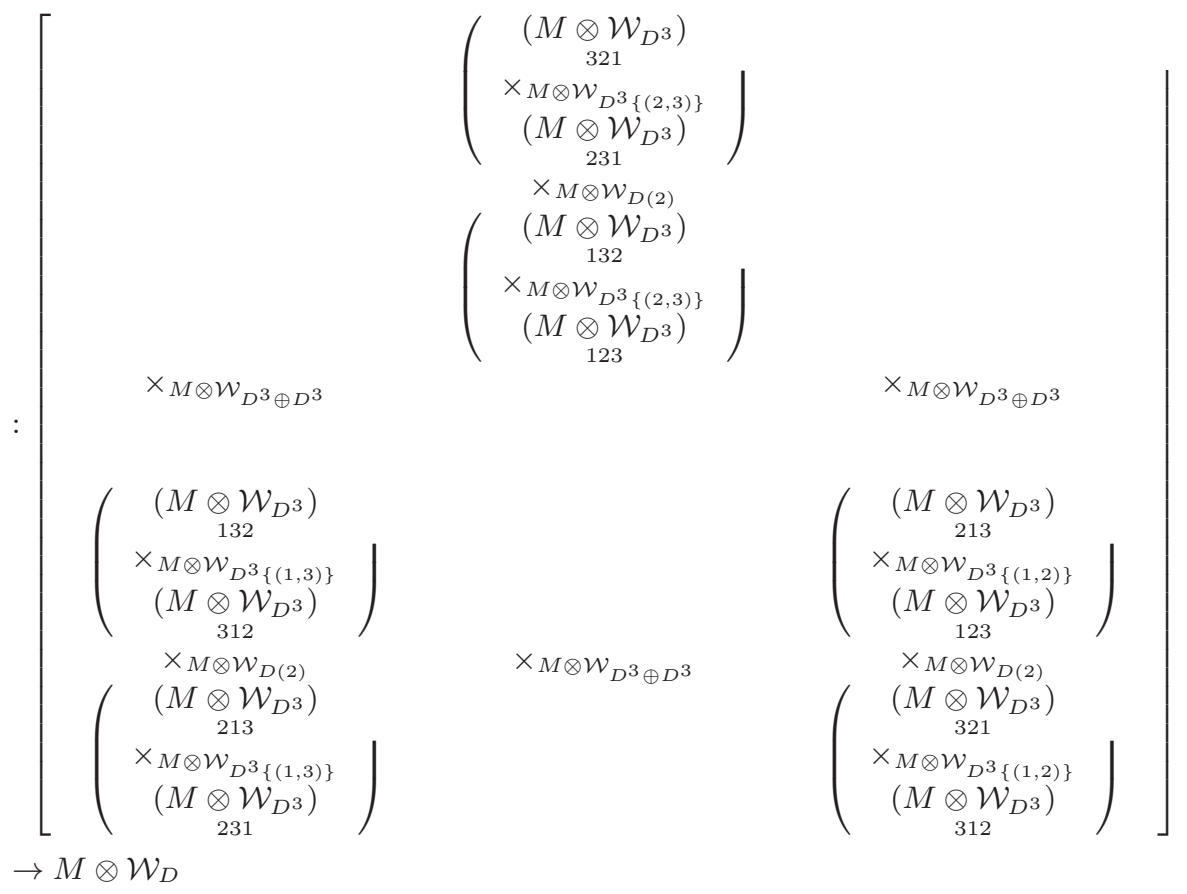

is equivalent to

$$
\begin{aligned}
& \left(\operatorname{id}_{M} \otimes \mathcal{W}_{d \in D \mapsto(d, d, d) \in D(3)}\right) \\
& \circ\left(\operatorname{id}_{M} \otimes \mathcal{W}_{\left(d_{1}, d_{2}, d_{3}\right) \in D(3) \mapsto\left(0,0,0,0,0,0, d_{1}-d_{3}, d_{2}-d_{3}\right) \in G}\right) \\
= & \operatorname{id}_{M} \otimes\left(\mathcal{W}_{d \in D \mapsto(d, d, d) \in D(3)} \circ \mathcal{W}_{\left(d_{1}, d_{2}, d_{3}\right) \in D(3) \mapsto\left(0,0,0,0,0,0, d_{1}-d_{3}, d_{2}-d_{3}\right) \in G}\right) \\
= & \operatorname{id}_{M} \otimes \mathcal{W}_{d \in D \mapsto(0,0,0,0,0,0,0,0) \in G}
\end{aligned}
$$

This completes the proof.

\section{From the General Jacobi Identity to the Jacobi Identity}

Notation 35. We write

$$
\left(M^{M} \otimes \mathcal{W}_{D}\right)_{\operatorname{id}_{M}}
$$

for the pullback of

$$
\begin{array}{ccc}
\left(M^{M} \otimes \mathcal{W}_{D}\right)_{\operatorname{id}_{M}} & \rightarrow & M^{M} \otimes \mathcal{W}_{D} \\
\downarrow & & \downarrow \\
1 & \rightarrow & M^{M}
\end{array}
$$


where the right arrow $M^{M} \otimes \mathcal{W}_{D} \rightarrow M^{M}$ is the canonical projection, while the bottom arrow is the exponential transpose of $\operatorname{id}_{M}: 1 \times M=M \rightarrow M$.

Theorem 36. The composition of morphisms

$$
\begin{gathered}
\left(M_{1}^{M} \otimes \mathcal{W}_{D}\right)_{\mathrm{id}_{M}} \times\left(M_{2}^{M} \underset{2}{\otimes} \mathcal{W}_{D}\right)_{\mathrm{id}_{M}} \stackrel{\operatorname{Ass}_{M}^{1,1}}{\longrightarrow}\left(M^{M} \otimes \mathcal{W}_{D^{2}}\right)_{\mathrm{id}_{M}} \\
M^{M} \otimes \mathcal{W}_{D^{2}} \rightarrow M^{M} \otimes \mathcal{W}_{D(2)}
\end{gathered}
$$

in succession is equivalent to the composition of morphisms

$$
\begin{aligned}
& \left(\begin{array}{c}
M^{M} \otimes \mathcal{W}_{D} \\
1
\end{array}\right)_{\mathrm{id}_{M}} \times\left(\begin{array}{c}
M^{M} \otimes \mathcal{W}_{D} \\
2
\end{array}\right)_{\mathrm{id}_{M}} \\
& \rightarrow\left(\begin{array}{c}
M^{M} \otimes \mathcal{W}_{D} \\
2
\end{array}\right)_{\mathrm{id}_{M}} \times\left(M^{M} \otimes \mathcal{W}_{D}\right)_{\mathrm{id}_{M}} \\
& \left.\stackrel{\operatorname{Ass}_{M}^{1,1}}{\stackrel{\mathrm{id}_{M^{M}}^{M}}{\longrightarrow} \otimes \mathcal{W}_{\left(d_{1}, d_{2}\right) \in D^{2} \mapsto\left(d_{2}, d_{1}\right) \in D^{2}}}\left(M^{M} \otimes \mathcal{W}_{D^{2}}\right)_{\mathrm{id}_{M}}\right)_{\mathrm{id}_{M}} \\
& \quad\left(M^{M} \otimes \mathcal{W}_{D^{2}}\right)_{\mathrm{id}_{M}} \rightarrow\left(M^{M} \otimes \mathcal{W}_{D(2)}\right)_{\mathrm{id}_{M}}
\end{aligned}
$$

in succession, so that we have

$$
\begin{aligned}
& \left(M_{1}^{M} \otimes \mathcal{W}_{D}\right)_{\operatorname{id}_{M}} \times\left(M_{2}^{M} \underset{2}{ } \mathcal{W}_{D}\right)_{\mathrm{id}_{M}} \stackrel{((30),(29))}{\longrightarrow} \\
& \left(M^{M} \otimes \mathcal{W}_{D^{2}}\right)_{\mathrm{id}_{M}} \times_{M^{M} \otimes \mathcal{W}_{D(2)}}\left(M^{M} \otimes \mathcal{W}_{D^{2}}\right)_{\mathrm{id}_{M}} \stackrel{\zeta^{-}}{\rightarrow}\left(M^{M} \otimes \mathcal{W}_{D}\right)_{\mathrm{id}_{M}}
\end{aligned}
$$

which is equivalent to

$$
L_{M}:\left(M_{1}^{M} \otimes \mathcal{W}_{D}\right)_{\mathrm{id}_{M}} \times\left(M_{2}^{M} \underset{\mathcal{W}_{D}}{ }\right)_{\mathrm{id}_{M}} \rightarrow\left(M^{M} \otimes \mathcal{W}_{D}\right)_{\mathrm{id}_{M}}
$$

Proof. The nontrivial part of the statement is only the equivalence of (31) and (32), for which it is easy to modify the proof of Proposition 8 in $§ 3.4$ of $[1]$.

The following proposition should be obvious.

Proposition 37. We have the following two statements. 
1. The composition of morphisms

$$
\begin{gathered}
\left(\left(\begin{array}{c}
M^{M} \otimes \mathcal{W}_{D^{2}} \\
1
\end{array}\right)_{\mathrm{id}_{M}} \times_{M^{M}} \otimes \mathcal{W}_{D(2)}\left(M_{2}^{M} \otimes \mathcal{W}_{D^{2}}\right)_{\mathrm{id}_{M}}\right) \times\left(M^{M} \otimes \mathcal{W}_{D}\right)_{\mathrm{id}_{M}} \\
\rightarrow\left(\begin{array}{c}
M^{M} \otimes \mathcal{W}_{D^{2}} \\
1
\end{array}\right)_{\mathrm{id}_{M}} \times\left(M^{M} \otimes \mathcal{W}_{D}\right)_{\mathrm{id}_{M}} \stackrel{\operatorname{Ass}_{M}^{2,1}}{\longrightarrow}\left(M^{M} \otimes \mathcal{W}_{D^{3}}\right)_{\mathrm{id}_{M}}(33) \\
\left(M^{M} \otimes \mathcal{W}_{D^{3}}\right)_{\mathrm{id}_{M}} \rightarrow\left(M^{M} \otimes \mathcal{W}_{D^{3}\{(1,2)\}}\right)_{\mathrm{id}_{M}}
\end{gathered}
$$

in succession is equivalent to the composition of morphisms

$$
\begin{gathered}
\left(\left(M^{M} \underset{1}{\otimes} \mathcal{W}_{D^{2}}\right)_{\mathrm{id}_{M}} \times_{M^{M}} \otimes \mathcal{W}_{D(2)}\left(M^{M} \underset{2}{\otimes} \mathcal{W}_{D^{2}}\right)_{\mathrm{id}_{M}}\right) \times\left(M^{M} \otimes \mathcal{W}_{D}\right)_{\mathrm{id}_{M}} \\
\rightarrow\left(\begin{array}{c}
M^{M} \otimes \mathcal{W}_{D^{2}} \\
2
\end{array}\right)_{\mathrm{id}_{M}} \times\left(M^{M} \otimes \mathcal{W}_{D}\right)_{\mathrm{id}_{M}} \stackrel{\operatorname{Ass}_{M}^{2,1}}{\longrightarrow}\left(M^{M} \otimes \mathcal{W}_{D^{3}}\right)_{\mathrm{id}_{M}}(34) \\
\left(M^{M} \otimes \mathcal{W}_{D^{3}}\right)_{\mathrm{id}_{M}} \rightarrow\left(M^{M} \otimes \mathcal{W}_{D^{3}\{(1,2)\}}\right)_{\mathrm{id}_{M}}
\end{gathered}
$$

in succession, so that we have the morphism

$$
\begin{aligned}
& \left(\left(\begin{array}{c}
M^{M} \otimes \mathcal{W}_{D^{2}} \\
1
\end{array}\right)_{\mathrm{id}_{M}} \times_{M^{M}} \otimes \mathcal{W}_{D(2)}\left(M^{M} \otimes \mathcal{W}_{D^{2}}\right)_{\mathrm{id}_{M}}\right) \times\left(M^{M} \otimes \mathcal{W}_{D}\right)_{\mathrm{id}_{M}} \\
& \stackrel{((33),(34))}{\longrightarrow}\left(M^{M} \otimes \mathcal{W}_{D^{3}}\right)_{\mathrm{id}_{M}} \times_{M^{M} \otimes \mathcal{W}_{D^{3}\{(1,2)\}}}\left(M^{M} \otimes \mathcal{W}_{D^{3}}\right)_{\mathrm{id}_{M}} \\
& \quad \stackrel{\zeta^{\frac{5}{\longrightarrow}}}{\longrightarrow}\left(M^{M} \otimes \mathcal{W}_{D^{2}}\right)_{\mathrm{id}_{M}}
\end{aligned}
$$

which is equivalent to the morphism

$$
\begin{gathered}
\left(\left(M^{M} \otimes \mathcal{W}_{D^{2}}\right)_{\mathrm{id}_{M}} \times_{M^{M} \otimes \mathcal{W}_{D(2)}}\left(M^{M} \underset{2}{\otimes} \mathcal{W}_{D^{2}}\right)_{\mathrm{id}_{M}}\right) \times\left(M^{M} \otimes \mathcal{W}_{D}\right)_{\mathrm{id}_{M}} \\
\stackrel{\stackrel{\zeta^{-}}{ } \times \operatorname{id}_{M^{M} \otimes \mathcal{W}_{D}}}{\longrightarrow}\left(M^{M} \otimes \mathcal{W}_{D}\right)_{\mathrm{id}_{M}} \times\left(M^{M} \otimes \mathcal{W}_{D}\right)_{\mathrm{id}_{M}} \stackrel{\operatorname{Ass}_{M}^{1,1}}{\longrightarrow}\left(M^{M} \otimes \mathcal{W}_{D^{2}}\right)_{\mathrm{id}_{M}}
\end{gathered}
$$

2. The composition of morphisms

$$
\begin{gathered}
\left(M^{M} \otimes \mathcal{W}_{D}\right)_{\operatorname{id}_{M}} \times\left(\left(M^{M} \underset{1}{\otimes} \mathcal{W}_{D^{2}}\right)_{\mathrm{id}_{M}} \times \times_{M^{M}} \otimes \mathcal{W}_{D(2)}\left(M_{2}^{M} \underset{D^{2}}{\otimes} \mathcal{W}_{\mathrm{id}_{M}}\right)\right. \\
\rightarrow\left(M^{M} \otimes \mathcal{W}_{D}\right)_{\mathrm{id}_{M}} \times\left(\begin{array}{c}
M^{M} \otimes \mathcal{W}_{D^{2}} \\
1
\end{array}\right)_{\mathrm{id}_{M}} \stackrel{\operatorname{Ass}_{M}^{1,2}}{\longrightarrow}\left(M^{M} \otimes \mathcal{W}_{D^{3}}\right)_{\mathrm{id}_{M}}
\end{gathered}
$$




$$
\left(M^{M} \otimes \mathcal{W}_{D^{3}}\right)_{\mathrm{id}_{M}} \rightarrow\left(M^{M} \otimes \mathcal{W}_{D^{3}\{(2,3)\}}\right)_{\mathrm{id}_{M}}
$$

in succession is equivalent to the composition of morphisms

$$
\begin{gathered}
\left(M^{M} \otimes \mathcal{W}_{D}\right)_{\mathrm{id}_{M}} \times\left(\left(M^{M} \underset{\mathcal{W}_{D^{2}}}{\otimes}\right)_{\mathrm{id}_{M}} \times_{M^{M} \otimes \mathcal{W}_{D(2)}}\left(M_{2}^{M} \underset{\mathcal{W}_{D^{2}}}{ }\right)_{\mathrm{id}_{M}}\right) \\
\rightarrow\left(M^{M} \otimes \mathcal{W}_{D}\right)_{\mathrm{id}_{M}} \times\left(M_{2}^{M} \underset{2}{\otimes} \mathcal{W}_{D^{2}}\right)_{\mathrm{id}_{M}} \stackrel{\operatorname{Ass}_{M}^{1,2}}{\longrightarrow}\left(M^{M} \otimes \mathcal{W}_{D^{3}}\right)_{\mathrm{id}_{M}} \\
\left(M^{M} \otimes \mathcal{W}_{D^{3}}\right)_{\mathrm{id}_{M}} \rightarrow\left(M^{M} \otimes \mathcal{W}_{D^{3}\{(2,3)\}}\right)_{\mathrm{id}_{M}}
\end{gathered}
$$

in succession, so that we have the morphism

$$
\begin{aligned}
& \left(M^{M} \otimes \mathcal{W}_{D}\right)_{\mathrm{id}_{M}} \times\left(\left(M^{M} \underset{1}{\otimes} \mathcal{W}_{D^{2}}\right)_{\mathrm{id}_{M}} \times_{M^{M} \otimes \mathcal{W}_{D(2)}}\left(M_{2}^{M}{\underset{2}{D^{2}}}_{\mathrm{id}_{M}}\right)\right. \\
& \stackrel{((35),(36))}{\longrightarrow}\left(M^{M} \otimes \mathcal{W}_{D^{3}}\right)_{\mathrm{id}_{M}} \times_{M^{M} \otimes \mathcal{W}_{D^{3}\{(2,3)\}}}\left(M^{M} \otimes \mathcal{W}_{D^{3}}\right)_{\mathrm{id}_{M}} \\
& \stackrel{\dot{\zeta^{1}}}{\longrightarrow}\left(M^{M} \otimes \mathcal{W}_{D^{2}}\right)_{\mathrm{id}_{M}}
\end{aligned}
$$

which is equivalent to the morphism

$$
\begin{aligned}
& \left(M^{M} \otimes \mathcal{W}_{D}\right)_{\mathrm{id}_{M}} \times\left(\left(M^{M} \underset{1}{\otimes} \mathcal{W}_{D^{2}}\right)_{\mathrm{id}_{M}} \times_{M^{M} \otimes \mathcal{W}_{D(2)}}\left(M^{M} \underset{2}{\otimes} \mathcal{W}_{D^{2}}\right)_{\mathrm{id}_{M}}\right) \\
& \underline{\zeta^{-} \times \operatorname{id}_{M^{M} \otimes \mathcal{W}_{D}}} \times\left(M^{M} \otimes \mathcal{W}_{D}\right)_{\mathrm{id}_{M}} \times\left(M^{M} \otimes \mathcal{W}_{D}\right)_{\mathrm{id}_{M}} \\
& \underset{\operatorname{Ass}_{M}^{1,1}}{\longrightarrow}\left(M^{M} \otimes \mathcal{W}_{D^{2}}\right)_{\mathrm{id}_{M}}
\end{aligned}
$$

Notation 38. We introduce the following fifteen morphisms:

1.

$$
\begin{aligned}
& \chi^{*_{1}}:\left(M^{M} \otimes \mathcal{W}_{D}\right)_{\operatorname{id}_{M}} \times\left(M^{M} \underset{2}{\otimes} \mathcal{W}_{D}\right)_{\mathrm{id}_{M}} \times\left(M_{3}^{M} \underset{3}{\otimes} \mathcal{W}_{D}\right)_{\mathrm{id}_{M}} \rightarrow \\
& \left(M^{M} \otimes \mathcal{W}_{D}\right)_{\mathrm{id}_{M}}
\end{aligned}
$$

as the canonical projection. 
2.

$$
\begin{aligned}
& \chi^{*_{2}}:\left(M^{M} \otimes \mathcal{W}_{D}\right)_{\mathrm{id}_{M}} \times\left(M_{2}^{M} \otimes \mathcal{W}_{D}\right)_{\mathrm{id}_{M}} \times\left(\begin{array}{c}
M^{M} \underset{3}{\otimes} \mathcal{W}_{D} \\
)_{\mathrm{id}_{M}}
\end{array} \rightarrow\right. \\
& \left(M_{2}^{M} \underset{2}{\otimes} \mathcal{W}_{D}\right)_{\mathrm{id}_{M}}
\end{aligned}
$$

as the canonical projection.

3.

$$
\begin{aligned}
\chi^{* 3}: & \left(M^{M} \otimes \mathcal{W}_{D}\right)_{\operatorname{id}_{M}} \times\left(M^{M} \underset{2}{\otimes} \mathcal{W}_{D}\right)_{\mathrm{id}_{M}} \times\left(M_{3}^{M} \underset{3}{\otimes} \mathcal{W}_{D}\right)_{\mathrm{id}_{M}} \rightarrow \\
& \left(\begin{array}{c}
M^{M} \otimes \mathcal{W}_{D} \\
3
\end{array}\right)_{\mathrm{id}_{M}}
\end{aligned}
$$

as the canonical projection.

4.

$$
\begin{aligned}
\chi^{* 12} & :\left(M^{M} \otimes \mathcal{W}_{D}\right)_{\operatorname{id}_{M}} \times\left(M_{2}^{M} \otimes \mathcal{W}_{D}\right)_{\mathrm{id}_{M}} \times\left(M_{3}^{M} \underset{3}{\otimes} \mathcal{W}_{D}\right)_{\operatorname{id}_{M}} \\
& \rightarrow\left(M^{M} \otimes \mathcal{W}_{D^{2}}\right)_{\mathrm{id}_{M}}
\end{aligned}
$$

as

$$
\begin{aligned}
& \left(\begin{array}{c}
M_{1}^{M} \otimes \mathcal{W}_{D} \\
1
\end{array}\right)_{\mathrm{id}_{M}} \times\left(\begin{array}{c}
M^{M} \otimes \mathcal{W}_{D} \\
2
\end{array}\right)_{\mathrm{id}_{M}} \times\left(\begin{array}{c}
M^{M} \otimes \mathcal{W}_{D} \\
3
\end{array}\right)_{\mathrm{id}_{M}} \\
& \rightarrow\left(\begin{array}{c}
M^{M} \otimes \mathcal{W}_{D} \\
1
\end{array}\right)_{\mathrm{id}_{M}} \times\left(\begin{array}{c}
M^{M} \otimes \mathcal{W}_{D} \\
2
\end{array}\right)_{\mathrm{id}_{M}} \stackrel{\operatorname{Ass}_{M}^{1,1}}{\longrightarrow}\left(M^{M} \otimes \mathcal{W}_{D^{2}}\right)_{\mathrm{id}_{M}}
\end{aligned}
$$

5.

$$
\begin{aligned}
\chi^{* 21} & :\left(M^{M} \otimes \mathcal{W}_{D}\right)_{\operatorname{id}_{M}} \times\left(M^{M} \otimes \mathcal{W}_{D}\right. \\
\quad & )_{\mathrm{id}_{M}} \times\left(M_{3}^{M} \otimes \mathcal{W}_{D}\right)_{\mathrm{id}_{M}} \\
& \rightarrow\left(M^{M} \otimes \mathcal{W}_{D^{2}}\right)_{\mathrm{id}_{M}}
\end{aligned}
$$

as

$$
\begin{aligned}
& \left(\begin{array}{c}
M^{M} \otimes \mathcal{W}_{D} \\
1
\end{array}\right)_{\mathrm{id}_{M}} \times\left(\begin{array}{c}
M^{M} \underset{2}{\otimes} \mathcal{W}_{D} \\
\mathrm{id}_{M}
\end{array} \times\left(\begin{array}{c}
M^{M} \otimes \mathcal{W}_{D} \\
3
\end{array}\right)_{\mathrm{id}_{M}}\right. \\
& \rightarrow\left(\begin{array}{c}
M^{M} \otimes \mathcal{W}_{D} \\
2
\end{array}\right)_{\mathrm{id}_{M}} \times\left(\begin{array}{c}
M^{M} \otimes \mathcal{W}_{D} \\
1
\end{array}\right)_{\mathrm{id}_{M}} \stackrel{\operatorname{Ass}_{M}^{1,1}}{\longrightarrow}\left(M^{M} \otimes \mathcal{W}_{D^{2}}\right)_{\mathrm{id}_{M}}
\end{aligned}
$$

$\underset{\operatorname{id}_{M^{M}} \otimes \mathcal{W}_{\left(d_{1}, d_{2}\right) \in D^{2} \mapsto\left(d_{2}, d_{1}\right) \in D^{2}}^{\longrightarrow}}{\longrightarrow}\left(M^{M} \otimes \mathcal{W}_{D^{2}}\right)_{\operatorname{id}_{M}}$ 
6.

$$
\begin{aligned}
\chi^{* 13} & :\left(M^{M} \otimes \mathcal{W}_{D}\right)_{\mathrm{id}_{M}} \times\left(M_{2}^{M} \otimes \mathcal{W}_{D}\right)_{\mathrm{id}_{M}} \times\left(M_{3}^{M} \underset{3}{\otimes} \mathcal{W}_{D}\right)_{\mathrm{id}_{M}} \\
& \rightarrow\left(M^{M} \otimes \mathcal{W}_{D^{2}}\right)_{\mathrm{id}_{M}}
\end{aligned}
$$

as

$$
\begin{aligned}
& \left(M_{1}^{M} \underset{1}{\otimes} \mathcal{W}_{D}\right)_{\operatorname{id}_{M}} \times\left(M^{M} \underset{2}{\otimes} \mathcal{W}_{D}\right)_{\operatorname{id}_{M}} \times\left(\begin{array}{c}
M^{M} \underset{3}{\otimes} \mathcal{W}_{D} \\
\operatorname{id}_{M} \\
\end{array}\right. \\
& \rightarrow\left(M^{M} \otimes \mathcal{W}_{D}\right)_{\mathrm{id}_{M}} \times\left(M^{M} \underset{3}{\otimes} \mathcal{W}_{D}\right)_{\mathrm{id}_{M}} \stackrel{\operatorname{Ass}_{M}^{1,1}}{\longrightarrow}\left(M^{M} \otimes \mathcal{W}_{D^{2}}\right)_{\mathrm{id}_{M}}
\end{aligned}
$$

7.

$$
\begin{aligned}
\chi^{* 31} & :\left(M^{M} \otimes \mathcal{W}_{D}\right)_{\mathrm{id}_{M}} \times\left(M^{M} \underset{2}{\otimes} \mathcal{W}_{D}\right)_{\mathrm{id}_{M}} \times\left(M_{3}^{M} \otimes \mathcal{W}_{D}\right)_{\mathrm{id}_{M}} \\
& \rightarrow\left(M^{M} \otimes \mathcal{W}_{D^{2}}\right)_{\mathrm{id}_{M}}
\end{aligned}
$$

as

$$
\begin{aligned}
& \left(\begin{array}{c}
M^{M} \otimes \mathcal{W}_{D} \\
1
\end{array}\right)_{\mathrm{id}_{M}} \times\left(\begin{array}{c}
M^{M} \otimes \mathcal{W}_{D} \\
2
\end{array}\right)_{\mathrm{id}_{M}} \times\left(\begin{array}{c}
M^{M} \otimes \mathcal{W}_{D} \\
3
\end{array}\right)_{\mathrm{id}_{M}} \\
& \rightarrow\left(\begin{array}{c}
M^{M} \otimes \mathcal{W}_{D} \\
3
\end{array}\right)_{\mathrm{id}_{M}} \times\left(\begin{array}{c}
M^{M} \otimes \mathcal{W}_{D} \\
1
\end{array}\right)_{\mathrm{id}_{M}} \stackrel{\operatorname{Ass}_{M}^{1,1} \longrightarrow}{\longrightarrow}\left(M^{M} \otimes \mathcal{W}_{D^{2}}\right)_{\mathrm{id}_{M}} \\
& \stackrel{\operatorname{id}_{M^{M}} \otimes \mathcal{W}_{\left(d_{1}, d_{2}\right) \in D^{2} \mapsto\left(d_{2}, d_{1}\right) \in D^{2}}^{\longrightarrow}}{\longrightarrow}\left(M^{M} \otimes \mathcal{W}_{D^{2}}\right)_{\mathrm{id}_{M}}
\end{aligned}
$$

8.

$$
\begin{aligned}
\chi^{* 23} & :\left(M^{M} \otimes \mathcal{W}_{D}\right)_{\operatorname{id}_{M}} \times\left(M^{M} \underset{2}{\otimes} \mathcal{W}_{D}\right)_{\mathrm{id}_{M}} \times\left(M^{M} \underset{3}{\otimes} \mathcal{W}_{D}\right)_{\mathrm{id}_{M}} \\
& \rightarrow\left(M^{M} \otimes \mathcal{W}_{D^{2}}\right)_{\mathrm{id}_{M}}
\end{aligned}
$$

as

$$
\begin{aligned}
& \left(\begin{array}{c}
M^{M} \otimes \mathcal{W}_{D} \\
1
\end{array}\right)_{\mathrm{id}_{M}} \times\left(\begin{array}{c}
M^{M} \otimes \mathcal{W}_{D} \\
2
\end{array}\right)_{\mathrm{id}_{M}} \times\left(\begin{array}{c}
M^{M} \otimes \mathcal{W}_{D} \\
3
\end{array}\right)_{\operatorname{id}_{M}} \\
& \rightarrow\left(\begin{array}{c}
M^{M} \otimes \mathcal{W}_{D} \\
2
\end{array}\right)_{\operatorname{id}_{M}} \times\left(\begin{array}{c}
M \\
\otimes \\
3
\end{array} \mathcal{W}_{D}\right)_{\mathrm{id}_{M}} \stackrel{\operatorname{Ass}_{M}^{1,1}}{\longrightarrow}\left(M^{M} \otimes \mathcal{W}_{D^{2}}\right)_{\mathrm{id}_{M}}
\end{aligned}
$$


9.

$$
\begin{aligned}
& \chi^{* 32}:\left(M^{M} \otimes \mathcal{W}_{D}\right)_{\mathrm{id}_{M}} \times\left(M^{M} \underset{2}{\otimes} \mathcal{W}_{D}\right)_{\mathrm{id}_{M}} \times\left(\begin{array}{c}
M^{M} \underset{3}{\otimes} \mathcal{W}_{D} \\
)_{\mathrm{id}_{M}}
\end{array}\right. \\
& \rightarrow\left(M^{M} \otimes \mathcal{W}_{D^{2}}\right)_{\operatorname{id}_{M}}
\end{aligned}
$$

as

$$
\begin{aligned}
& \left(M_{1}^{M} \underset{1}{\otimes} \mathcal{W}_{D}\right)_{\operatorname{id}_{M}} \times\left(M^{M} \underset{2}{\otimes} \mathcal{W}_{D}\right)_{\mathrm{id}_{M}} \times\left(\begin{array}{c}
M^{M} \otimes \mathcal{W}_{D} \\
3
\end{array}\right)_{\operatorname{id}_{M}} \\
& \rightarrow\left(M^{M} \otimes \mathcal{W}_{D}\right)_{\mathrm{id}_{M}} \times\left(M^{M} \otimes \mathcal{W}_{D}\right)_{\mathrm{id}_{M}} \stackrel{\operatorname{Ass}_{M}^{1,1}}{\longrightarrow}\left(M^{M} \otimes \mathcal{W}_{D^{2}}\right)_{\mathrm{id}_{M}} \\
& \underset{\operatorname{id}_{M^{M}} \otimes \mathcal{W}_{\left(d_{1}, d_{2}\right) \in D^{2} \mapsto\left(d_{2}, d_{1}\right) \in D^{2}}^{\longrightarrow}}{\longrightarrow}\left(M^{M} \otimes \mathcal{W}_{D^{2}}\right)_{\operatorname{id}_{M}}
\end{aligned}
$$

10.

$$
\begin{aligned}
\chi^{* 123} & :\left(M^{M} \otimes \mathcal{W}_{D}\right)_{\mathrm{id}_{M}} \times\left(M^{M} \underset{2}{\otimes} \mathcal{W}_{D}\right)_{\mathrm{id}_{M}} \times\left(\begin{array}{c}
M^{M} \otimes \mathcal{W}_{D} \\
3
\end{array}\right)_{\mathrm{id}_{M}} \\
& \rightarrow\left(M^{M} \otimes \mathcal{W}_{D^{3}}\right)_{\mathrm{id}_{M}}
\end{aligned}
$$

as

$$
\begin{aligned}
& \operatorname{Ass}_{M}^{1,1,1}:\left(M_{1}^{M} \otimes \mathcal{W}_{D}\right)_{\operatorname{id}_{M}} \times\left(M_{2}^{M} \underset{2}{\otimes} \mathcal{W}_{D}\right)_{\mathrm{id}_{M}} \times\left(\begin{array}{c}
M^{M} \underset{3}{\otimes} \mathcal{W}_{D} \\
\operatorname{id}_{M} \\
\end{array}\right. \\
& \rightarrow\left(M^{M} \otimes \mathcal{W}_{D^{3}}\right)_{\mathrm{id}_{M}}
\end{aligned}
$$

11.

$$
\begin{aligned}
\chi^{* 132} & :\left(M^{M} \otimes \mathcal{W}_{D}\right)_{\mathrm{id}_{M}} \times\left(M^{M} \underset{2}{\otimes} \mathcal{W}_{D}\right)_{\mathrm{id}_{M}} \times\left(M_{3}^{M} \otimes \mathcal{W}_{D}\right)_{\mathrm{id}_{M}} \\
& \rightarrow\left(M^{M} \otimes \mathcal{W}_{D^{3}}\right)_{\mathrm{id}_{M}}
\end{aligned}
$$

as

$$
\begin{aligned}
& \left(M_{1}^{M} \otimes \mathcal{W}_{D}\right)_{\operatorname{id}_{M}} \times\left(M^{M} \underset{2}{\otimes} \mathcal{W}_{D}\right)_{\mathrm{id}_{M}} \times\left(\begin{array}{c}
M^{M} \otimes \mathcal{W}_{D} \\
3
\end{array}\right)_{\mathrm{id}_{M}} \\
& \rightarrow\left(M^{M} \otimes \mathcal{W}_{D}\right)_{\mathrm{id}_{M}} \times\left(M^{M} \underset{3}{\otimes} \mathcal{W}_{D}\right)_{\mathrm{id}_{M}} \times\left(M_{2}^{M} \otimes \mathcal{W}_{D}\right)_{\mathrm{id}_{M}} \stackrel{\operatorname{Ass}_{M}^{1,1,1}}{\longrightarrow} \\
& \left(M^{M} \otimes \mathcal{W}_{D^{3}}\right)_{\operatorname{id}_{M}} \underset{\operatorname{id}_{M^{M}} \otimes \mathcal{W}_{\left(d_{1}, d_{2}, d_{3}\right) \in D^{3} \mapsto\left(d_{1}, d_{3}, d_{2}\right) \in D^{3}}^{\longrightarrow}}{\longrightarrow}\left(M^{M} \otimes \mathcal{W}_{D^{3}}\right)_{\operatorname{id}_{M}}
\end{aligned}
$$


12.

$$
\begin{aligned}
\chi^{*_{213}} & :\left(M^{M} \otimes \mathcal{W}_{D}\right)_{\mathrm{id}_{M}} \times\left(M_{2}^{M} \otimes \mathcal{W}_{D}\right)_{\mathrm{id}_{M}} \times\left(M_{3}^{M} \otimes \mathcal{W}_{D}\right)_{\mathrm{id}_{M}} \\
& \rightarrow\left(M^{M} \otimes \mathcal{W}_{D^{3}}\right)_{\mathrm{id}_{M}}
\end{aligned}
$$

as

$$
\left.\begin{array}{l}
\left(\begin{array}{c}
M^{M} \otimes \mathcal{W}_{D} \\
1
\end{array}\right)_{\mathrm{id}_{M}} \times\left(\begin{array}{c}
M^{M} \otimes \mathcal{W}_{D} \\
2
\end{array}\right)_{\mathrm{id}_{M}} \times\left(M^{M} \otimes \mathcal{W}_{D}\right. \\
3
\end{array}\right)_{\mathrm{id}_{M}}
$$

13.

$$
\begin{aligned}
& \chi^{*_{231}}:\left(M^{M} \otimes \mathcal{W}_{D}\right)_{\mathrm{id}_{M}} \times\left(M_{2}^{M} \underset{2}{\otimes} \mathcal{W}_{D}\right)_{\mathrm{id}_{M}} \times\left(\begin{array}{c}
M^{M} \underset{3}{\otimes} \mathcal{W}_{D} \\
)_{\mathrm{id}_{M}}
\end{array}\right. \\
& \rightarrow\left(M^{M} \otimes \mathcal{W}_{D^{3}}\right)_{\mathrm{id}_{M}}
\end{aligned}
$$

as

$$
\begin{aligned}
& \left(M_{1}^{M} \otimes \mathcal{W}_{D}\right)_{\operatorname{id}_{M}} \times\left(\begin{array}{c}
M^{M} \underset{2}{\otimes} \mathcal{W}_{D} \\
)_{\mathrm{id}_{M}}
\end{array} \times\left(\begin{array}{c}
M^{M} \otimes \mathcal{W}_{D} \\
3
\end{array}\right)_{\operatorname{id}_{M}}\right. \\
& \rightarrow\left(M^{M} \underset{2}{\otimes} \mathcal{W}_{D}\right)_{\operatorname{id}_{M}} \times\left(M^{M} \underset{3}{\otimes \mathcal{W}_{D}}\right)_{\operatorname{id}_{M}} \times\left(M^{M} \underset{1}{\otimes \mathcal{W}_{D}}\right)_{\mathrm{id}_{M}} \stackrel{\operatorname{Ass}_{M}^{1,1,1}}{\longrightarrow} \\
& \left(M^{M} \otimes \mathcal{W}_{D^{3}}\right)_{\operatorname{id}_{M}} \underset{\operatorname{id}_{M^{M}} \otimes \mathcal{W}_{\left(d_{1}, d_{2}, d_{3}\right) \in D^{3} \mapsto\left(d_{2}, d_{3}, d_{1}\right) \in D^{3}}^{\longrightarrow}}{\longrightarrow}\left(M^{M} \otimes \mathcal{W}_{D^{3}}\right)_{\operatorname{id}_{M}}
\end{aligned}
$$

14.

$$
\begin{aligned}
\chi^{* 312} & :\left(M^{M} \otimes \mathcal{W}_{D}\right)_{\mathrm{id}_{M}} \times\left(M_{2}^{M} \otimes \mathcal{W}_{D}\right)_{\mathrm{id}_{M}} \times\left(M_{3}^{M} \otimes \mathcal{W}_{D}\right)_{\mathrm{id}_{M}} \\
& \rightarrow\left(M^{M} \otimes \mathcal{W}_{D^{3}}\right)_{\mathrm{id}_{M}}
\end{aligned}
$$

as

$$
\begin{aligned}
& \left(M^{M} \otimes \mathcal{W}_{D}\right)_{\mathrm{id}_{M}} \times\left(M^{M} \underset{2}{\otimes} \mathcal{W}_{D}\right)_{\mathrm{id}_{M}} \times\left(\begin{array}{c}
M^{M} \otimes \mathcal{W}_{D} \\
3
\end{array}\right)_{\mathrm{id}_{M}} \\
& \rightarrow\left(M^{M} \underset{3}{\otimes} \mathcal{W}_{D}\right)_{\mathrm{id}_{M}} \times\left(M^{M} \otimes \mathcal{W}_{D}\right)_{\mathrm{id}_{M}} \times\left(M_{2}^{M} \otimes \mathcal{W}_{D}\right)_{\mathrm{id}_{M}} \stackrel{\operatorname{Ass}_{M}^{1,1,1}}{\longrightarrow} \\
& \left(M^{M} \otimes \mathcal{W}_{D^{3}}\right)_{\mathrm{id}_{M}} \stackrel{\operatorname{id}_{M^{M}} \otimes \mathcal{W}_{\left(d_{1}, d_{2}, d_{3}\right) \in D^{3} \mapsto\left(d_{3}, d_{1}, d_{2}\right) \in D^{3}}^{\longrightarrow}}{\longrightarrow}\left(M^{M} \otimes \mathcal{W}_{D^{3}}\right)_{\mathrm{id}_{M}}
\end{aligned}
$$


15.

$$
\begin{aligned}
\chi^{* 321} & :\left(M^{M} \otimes \mathcal{W}_{D}\right)_{\operatorname{id}_{M}} \times\left(M^{M} \underset{2}{\otimes} \mathcal{W}_{D}\right)_{\mathrm{id}_{M}} \times\left(M_{3}^{M} \underset{3}{\otimes} \mathcal{W}_{D}\right)_{\mathrm{id}_{M}} \\
& \rightarrow\left(M^{M} \otimes \mathcal{W}_{D^{3}}\right)_{\mathrm{id}_{M}}
\end{aligned}
$$

as

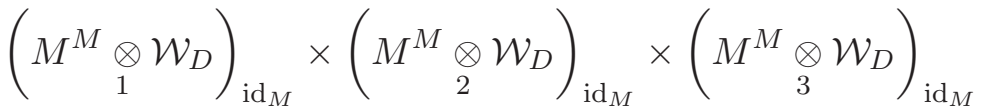

$$
\begin{aligned}
& \rightarrow\left(M_{3}^{M} \otimes \mathcal{W}_{D}\right)_{\mathrm{id}_{M}} \times\left(\begin{array}{c}
M^{M} \otimes \mathcal{W}_{D} \\
1
\end{array}\right)_{\mathrm{id}_{M}} \times\left(M_{2}^{M} \underset{\mathcal{W}_{D}}{\otimes}\right)_{\mathrm{id}_{M}} \stackrel{\operatorname{Ass}_{M}^{1,1,1}}{\longrightarrow} \\
& \left(M^{M} \otimes \mathcal{W}_{D^{3}}\right)_{\operatorname{id}_{M}} \stackrel{\operatorname{id}_{M^{M}} \otimes \mathcal{W}_{\left(d_{1}, d_{2}, d_{3}\right) \in D^{3} \mapsto\left(d_{3}, d_{1}, d_{2}\right) \in D^{3}}}{\longrightarrow}\left(M^{M} \otimes \mathcal{W}_{D^{3}}\right)_{\operatorname{id}_{M}}
\end{aligned}
$$

Lemma 39. We have the following statements:

1. The composition of morphisms $\chi^{* 321}$ and

$$
\left(M^{M} \otimes \mathcal{W}_{D^{3}}\right)_{\mathrm{id}_{M}} \rightarrow\left(M^{M} \otimes \mathcal{W}_{D^{3}\{(2,3)\}}\right)_{\mathrm{id}_{M}}
$$

is equivalent to the composition of morphisms $\chi^{* 231}$ and

$$
\left(M^{M} \otimes \mathcal{W}_{D^{3}}\right)_{\mathrm{id}_{M}} \rightarrow\left(M^{M} \otimes \mathcal{W}_{D^{3}\{(2,3)\}}\right)_{\mathrm{id}_{M}}
$$

so that we have

$$
\begin{gathered}
\left(M_{1}^{M} \otimes \mathcal{W}_{D}\right)_{\mathrm{id}_{M}} \times\left(M_{2}^{M} \underset{\mathcal{W}_{D}}{ }\right)_{\mathrm{id}_{M}} \times\left(M_{3}^{M} \underset{3}{\otimes} \mathcal{W}_{D}\right)_{\mathrm{id}_{M}} \\
\stackrel{\left(\chi^{* 321}, \chi^{*_{231}}\right)}{\longrightarrow}\left(M^{M} \otimes \mathcal{W}_{D^{3}}\right) \times_{M^{M} \otimes \mathcal{W}_{D^{3}\{(2,3)\}}}\left(M^{M} \otimes \mathcal{W}_{D^{3}}\right)_{\mathrm{id}_{M}} \stackrel{\zeta^{-}}{\longrightarrow}\left(M^{M} \otimes \mathcal{W}_{D^{2}}\right)_{\mathrm{id}_{M}}
\end{gathered}
$$

which is equivalent to

$$
\begin{aligned}
& \left(M_{1}^{M} \underset{1}{\otimes} \mathcal{W}_{D}\right)_{\operatorname{id}_{M}} \times\left(M^{M} \underset{2}{\otimes} \mathcal{W}_{D}\right)_{\mathrm{id}_{M}} \times\left(\begin{array}{c}
M^{M} \otimes \mathcal{W}_{D} \\
3
\end{array}\right)_{\operatorname{id}_{M}} \\
& \underline{\left(\left(\chi^{* 32}, \chi^{* 23}\right), \chi^{* 1}\right)} \\
& \left(\left(M^{M} \otimes \mathcal{W}_{D^{2}}\right)_{\mathrm{id}_{M}} \times_{M^{M}} \otimes \mathcal{W}_{D(2)}\left(M^{M} \otimes \mathcal{W}_{D^{2}}\right)_{\mathrm{id}_{M}}\right) \times\left(M^{M} \otimes \mathcal{W}_{D}\right)_{\operatorname{id}_{M}} \\
& \underline{\zeta^{-} \times \operatorname{id}_{M^{M} \otimes \mathcal{W}_{D}}}\left(M^{M} \otimes \mathcal{W}_{D}\right)_{\mathrm{id}_{M}} \times\left(M^{M} \otimes \mathcal{W}_{D}\right)_{\operatorname{id}_{M}}
\end{aligned}
$$




$$
\begin{aligned}
& \stackrel{\operatorname{Ass}_{M}^{1,1}}{\longrightarrow}\left(M^{M} \otimes \mathcal{W}_{D^{2}}\right)_{\mathrm{id}_{M}} \\
& \stackrel{\operatorname{id}_{M^{M}} \otimes \mathcal{W}_{\left(d_{1}, d_{2}\right) \in D^{2} \mapsto\left(d_{2}, d_{1}\right) \in D^{2}}}{\longrightarrow}\left(M^{M} \otimes \mathcal{W}_{D^{2}}\right)_{\mathrm{id}_{M}}
\end{aligned}
$$

2. The composition of morphisms $\chi^{*_{132}}$ and

$$
\left(M^{M} \otimes \mathcal{W}_{D^{3}}\right)_{\mathrm{id}_{M}} \rightarrow\left(M^{M} \otimes \mathcal{W}_{D^{3}\{(2,3)\}}\right)_{\mathrm{id}_{M}}
$$

is equivalent to the composition of morphisms $\chi^{*_{123}}$ and

$$
\left(M^{M} \otimes \mathcal{W}_{D^{3}}\right)_{\mathrm{id}_{M}} \rightarrow\left(M^{M} \otimes \mathcal{W}_{D^{3}\{(2,3)\}}\right)_{\mathrm{id}_{M}}
$$

so that we have

$$
\begin{aligned}
& \left(\begin{array}{c}
M^{M} \otimes \mathcal{W}_{D} \\
1
\end{array}\right)_{\mathrm{id}_{M}} \times\left(M^{M} \otimes \mathcal{W}_{D}\right)_{\mathrm{id}_{M}} \times\left(M^{M} \underset{3}{\otimes} \mathcal{W}_{D}\right)_{\mathrm{id}_{M}} \\
& \stackrel{\left(\chi^{* 132}, \chi^{* 123}\right)}{\longrightarrow}\left(M^{M} \otimes \mathcal{W}_{D^{3}}\right)_{\mathrm{id}_{M}} \times_{M^{M} \otimes \mathcal{W}_{D^{3}\{(2,3)\}}}\left(M^{M} \otimes \mathcal{W}_{D^{3}}\right)_{\mathrm{id}_{M}} \\
& \stackrel{\stackrel{\overline{1}}{\longrightarrow}}{\rightarrow}\left(M^{M} \otimes \mathcal{W}_{D^{2}}\right)_{\mathrm{id}_{M}}
\end{aligned}
$$

which is equivalent to

$$
\begin{aligned}
& \left(M^{M} \underset{1}{\otimes} \mathcal{W}_{D}\right)_{\operatorname{id}_{M}} \times\left(M^{M} \underset{2}{\otimes} \mathcal{W}_{D}\right)_{\mathrm{id}_{M}} \times\left(M_{3}^{M} \underset{3}{\otimes} \mathcal{W}_{D}\right)_{\operatorname{id}_{M}} \\
& \underline{\left(\chi^{* 1},\left(\chi^{*_{32}}, \chi^{*_{23}}\right)\right)} \\
& \left(M^{M} \otimes \mathcal{W}_{D}\right)_{\mathrm{id}_{M}} \times\left(\left(M^{M} \otimes \mathcal{W}_{D^{2}}\right)_{\mathrm{id}_{M}} \times_{M^{M} \otimes \mathcal{W}_{D(2)}}\left(M^{M} \otimes \mathcal{W}_{D^{2}}\right)_{\mathrm{id}_{M}}\right) \\
& \underset{\operatorname{id}_{M^{M} \otimes \mathcal{W}_{D}} \times \zeta^{-}}{\longrightarrow}\left(M^{M} \otimes \mathcal{W}_{D}\right)_{\operatorname{id}_{M}} \times\left(M^{M} \otimes \mathcal{W}_{D}\right)_{\mathrm{id}_{M}} \\
& \stackrel{\operatorname{Ass}_{M}^{1,1}}{\longrightarrow}\left(M^{M} \otimes \mathcal{W}_{D^{2}}\right)_{\mathrm{id}_{M}}
\end{aligned}
$$

3. The composition of (38) and

$$
\left(M^{M} \otimes \mathcal{W}_{D^{2}}\right)_{\mathrm{id}_{M}} \rightarrow\left(M^{M} \otimes \mathcal{W}_{D(2)}\right)_{\mathrm{id}_{M}}
$$

is equivalent to the composition of (40) and

$$
\left(M^{M} \otimes \mathcal{W}_{D^{2}}\right)_{\mathrm{id}_{M}} \rightarrow\left(M^{M} \otimes \mathcal{W}_{D(2)}\right)_{\mathrm{id}_{M}}
$$


so that we have

$$
\begin{aligned}
& \left(M_{1}^{M} \otimes \mathcal{W}_{D}\right)_{\mathrm{id}_{M}} \times\left(M_{2}^{M} \otimes \mathcal{W}_{D}\right)_{\mathrm{id}_{M}} \times\left(\begin{array}{c}
M_{3}^{M} \otimes \mathcal{W}_{D} \\
{ }_{\mathrm{id}_{M}}
\end{array}\right. \\
& \underline{\left(\left(\chi^{* 321}, \chi^{* 231}\right),\left(\chi^{* 132}, \chi^{* 123}\right)\right)} \\
& \left(\begin{array}{c}
\left(\begin{array}{c}
M^{M} \otimes \mathcal{W}_{D^{3}} \\
321
\end{array}\right)_{\mathrm{id}_{M}} \\
\times_{M^{M} \otimes \mathcal{W}_{D^{3}\{(2,3)\}}}\left(\begin{array}{c}
\left(M^{M} \otimes \mathcal{W}_{D^{3}}\right. \\
132
\end{array}\right)_{\mathrm{id}_{M}} \\
\left.\times_{M^{M} \otimes \mathcal{W}_{D^{3}\{(2,3)\}}} \otimes \mathcal{W}_{D^{3}}\right)_{\mathrm{id}_{M}}
\end{array}\right) \times_{M^{M} \otimes \mathcal{W}_{D(2)}}\left(\begin{array}{c}
\left.M^{M} \otimes \mathcal{W}_{D^{3}}\right)_{\mathrm{id}_{M}} \\
123
\end{array}\right) \\
& \stackrel{\zeta^{\overline{2}} \times M^{M} \otimes \mathcal{W}_{D(2)}}{\zeta^{\overline{2}}}\left(M^{M} \otimes \mathcal{W}_{D^{2}}\right)_{\mathrm{id}_{M}} \times_{M^{M} \otimes \mathcal{W}_{D(2)}}\left(M^{M} \otimes \mathcal{W}_{D^{2}}\right)_{\mathrm{id}_{M}} \\
& \stackrel{\zeta^{-}}{\rightarrow}\left(M^{M} \otimes \mathcal{W}_{D}\right)_{\operatorname{id}_{M}}
\end{aligned}
$$

which is equivalent to

$$
\begin{aligned}
& \left(\begin{array}{c}
M^{M} \otimes \mathcal{W}_{D} \\
1
\end{array}\right)_{\mathrm{id}_{M}} \times\left(M_{2}^{M} \otimes \mathcal{W}_{D}\right)_{\mathrm{id}_{M}} \times\left(M^{M} \otimes \mathcal{W}_{D}\right)_{\mathrm{id}_{M}} \\
& \stackrel{\operatorname{id}\left(\begin{array}{c}
M^{M} \otimes \mathcal{W}_{D} \\
1
\end{array}\right)_{\mathrm{id}_{M}} \times L_{M}}{\longrightarrow} \\
& \left(\begin{array}{c}
M^{M} \otimes \mathcal{W}_{D} \\
1
\end{array}\right)_{\mathrm{id}_{M}} \times\left(M^{M} \otimes \mathcal{W}_{D}\right)_{\mathrm{id}_{M}} \stackrel{L_{M}}{\longrightarrow}\left(M^{M} \otimes \mathcal{W}_{D}\right)_{\mathrm{id}_{M}}
\end{aligned}
$$

Proof. The first and the second statements follow from Proposition 37. The last statement follows from Theorem 36 .

Lemma 40. We have the following statements:

1. The composition of morphisms $\chi^{* 132}$ and

$$
\left(M^{M} \otimes \mathcal{W}_{D^{3}}\right)_{\mathrm{id}_{M}} \rightarrow\left(M^{M} \otimes \mathcal{W}_{D^{3}\{(1,3)\}}\right)_{\mathrm{id}_{M}}
$$

in succession is equivalent to the composition of morphisms $\chi^{* 312}$ and

$$
\left(M^{M} \otimes \mathcal{W}_{D^{3}}\right)_{\mathrm{id}_{M}} \rightarrow\left(M^{M} \otimes \mathcal{W}_{D^{3}\{(1,3)\}}\right)_{\mathrm{id}_{M}}
$$

in succession, so that we have

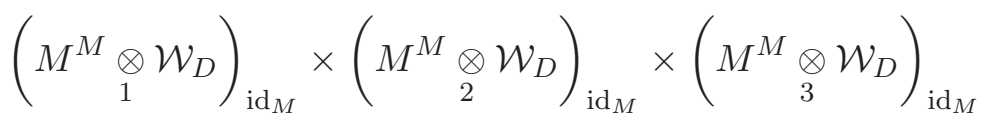




$$
\begin{aligned}
& \stackrel{\left(\chi^{* 132}, \chi^{* 312}\right)}{\longrightarrow}\left(M^{M} \otimes \mathcal{W}_{D^{3}}\right)_{\mathrm{id}_{M}} \times_{M^{M} \otimes \mathcal{W}_{D^{3}\{(1,3)\}}}\left(M^{M} \otimes \mathcal{W}_{D^{3}}\right)_{\mathrm{id}_{M}} \\
& \stackrel{\zeta^{\frac{-}{2}}}{\rightarrow}\left(M^{M} \otimes \mathcal{W}_{D^{2}}\right)_{\mathrm{id}_{M}}
\end{aligned}
$$

which is equivalent to

$$
\begin{aligned}
& \left(M_{1}^{M} \underset{\mathcal{W}_{D}}{\otimes}\right)_{\operatorname{id}_{M}} \times\left(\begin{array}{c}
M^{M} \underset{2}{\otimes} \mathcal{W}_{D} \\
)_{\mathrm{id}_{M}} \times\left(M^{M} \underset{3}{\otimes} \mathcal{W}_{D}\right.
\end{array}\right)_{\operatorname{id}_{M}} \\
& \underline{\left(\left(\chi^{* 13}, \chi^{* 31}\right), \chi^{* 2}\right)} \\
& \left(\left(M^{M} \otimes \mathcal{W}_{D^{2}}\right)_{\mathrm{id}_{M}} \times_{M^{M}} \otimes \mathcal{W}_{D(2)}\left(M^{M} \otimes \mathcal{W}_{D^{2}}\right)_{\mathrm{id}_{M}}\right) \times\left(M^{M} \otimes \mathcal{W}_{D}\right)_{\mathrm{id}_{M}} \\
& \stackrel{\zeta^{-} \times \operatorname{id}_{M^{M} \otimes \mathcal{W}_{D}}}{\longrightarrow}\left(M^{M} \otimes \mathcal{W}_{D}\right)_{\mathrm{id}_{M}} \times\left(\begin{array}{c}
M^{M} \otimes \mathcal{W}_{D} \\
2
\end{array}\right)_{\operatorname{id}_{M}} \\
& \underset{\operatorname{Ass}_{M}^{1,1}}{\longrightarrow}\left(M^{M} \otimes \mathcal{W}_{D^{2}}\right)_{\mathrm{id}_{M}} \\
& \stackrel{\operatorname{id}_{M^{M}} \otimes \mathcal{W}_{\left(d_{1}, d_{2}\right) \in D^{2} \mapsto\left(d_{2}, d_{1}\right) \in D^{2}}^{\longrightarrow}}{\longrightarrow}\left(M^{M} \otimes \mathcal{W}_{D^{2}}\right)_{\mathrm{id}_{M}}
\end{aligned}
$$

2. The composition of morphisms $\chi^{*_{213}}$ and

$$
\left(M^{M} \otimes \mathcal{W}_{D^{3}}\right)_{\mathrm{id}_{M}} \rightarrow\left(M^{M} \otimes \mathcal{W}_{D^{3}\{(1,3)\}}\right)_{\mathrm{id}_{M}}
$$

is equivalent to the composition of morphisms $\chi^{*_{231}}$ and

$$
\left(M^{M} \otimes \mathcal{W}_{D^{3}}\right)_{\operatorname{id}_{M}} \rightarrow\left(M^{M} \otimes \mathcal{W}_{D^{3}\{(1,3)\}}\right)_{\operatorname{id}_{M}}
$$

in succession, so that we have

$$
\begin{aligned}
& \left(M^{M} \underset{1}{\otimes} \mathcal{W}_{D}\right)_{\mathrm{id}_{M}} \times\left(\begin{array}{c}
M^{M} \underset{2}{\otimes} \mathcal{W}_{D} \\
)_{\mathrm{id}_{M}} \times\left(M^{M} \underset{3}{\otimes} \mathcal{W}_{D}\right.
\end{array}\right)_{\mathrm{id}_{M}} \\
& \stackrel{\left(\chi^{*_{213}}, \chi^{*_{231}}\right)}{\longrightarrow}\left(M^{M} \otimes \mathcal{W}_{D^{3}}\right)_{\mathrm{id}_{M}} \times_{M^{M} \otimes \mathcal{W}_{D^{3}\{(2,3)\}}}\left(M^{M} \otimes \mathcal{W}_{D^{3}}\right)_{\mathrm{id}_{M}} \\
& \underset{\zeta^{\overline{2}}}{\longrightarrow}\left(M^{M} \otimes \mathcal{W}_{D^{2}}\right)_{\mathrm{id}_{M}}
\end{aligned}
$$

which is equivalent to

$$
\begin{aligned}
& \left(\begin{array}{c}
M^{M} \otimes \mathcal{W}_{D} \\
1
\end{array}\right)_{\operatorname{id}_{M}} \times\left(M^{M} \underset{2}{\otimes} \mathcal{W}_{D}\right)_{\mathrm{id}_{M}} \times\left(M_{3}^{M} \underset{3}{\otimes} \mathcal{W}_{D}\right)_{\mathrm{id}_{M}} \\
& \underline{\left(\chi^{* 2},\left(\chi^{* 13}, \chi^{* 31}\right)\right)}
\end{aligned}
$$




$$
\begin{aligned}
& \left(M_{1}^{M} \otimes \mathcal{W}_{D}\right)_{\mathrm{id}_{M}} \times\left(\left(M^{M} \otimes \mathcal{W}_{D^{2}}\right)_{\mathrm{id}_{M}} \times M^{M} \otimes \mathcal{W}_{D(2)}\left(M^{M} \otimes \mathcal{W}_{D^{2}}\right)_{\mathrm{id}_{M}}\right) \\
& \stackrel{\operatorname{id}_{M^{M} \otimes \mathcal{W}_{D}} \times \zeta^{-}}{\longrightarrow}\left(M^{M} \otimes \mathcal{W}_{D}\right)_{\mathrm{id}_{M}} \times\left(M^{M} \otimes \mathcal{W}_{D}\right)_{\mathrm{id}_{M}} \\
& \stackrel{\operatorname{Ass}_{M}^{1,1}}{\longrightarrow}\left(M^{M} \otimes \mathcal{W}_{D^{2}}\right)_{\mathrm{id}_{M}}
\end{aligned}
$$

3. The composition of (44) and

$$
\left(M^{M} \otimes \mathcal{W}_{D^{2}}\right)_{\mathrm{id}_{M}} \rightarrow\left(M^{M} \otimes \mathcal{W}_{D(2)}\right)_{\mathrm{id}_{M}}
$$

is equivalent to the composition of (46) and

$$
\left(M^{M} \otimes \mathcal{W}_{D^{2}}\right)_{\mathrm{id}_{M}} \rightarrow\left(M^{M} \otimes \mathcal{W}_{D(2)}\right)_{\mathrm{id}_{M}}
$$

so that we have

$$
\begin{aligned}
& \left(M_{1}^{M} \otimes \mathcal{W}_{D}\right)_{\operatorname{id}_{M}} \times\left(M_{2}^{M} \underset{2}{\otimes} \mathcal{W}_{D}\right)_{\mathrm{id}_{M}} \times\left(\begin{array}{c}
M^{M} \underset{3}{\otimes} \mathcal{W}_{D} \\
\operatorname{id}_{M}
\end{array}\right. \\
& \underline{\left(\left(\chi^{* 132}, \chi^{* 312}\right),\left(\chi^{*_{213}}, \chi^{* 231}\right)\right)}
\end{aligned}
$$

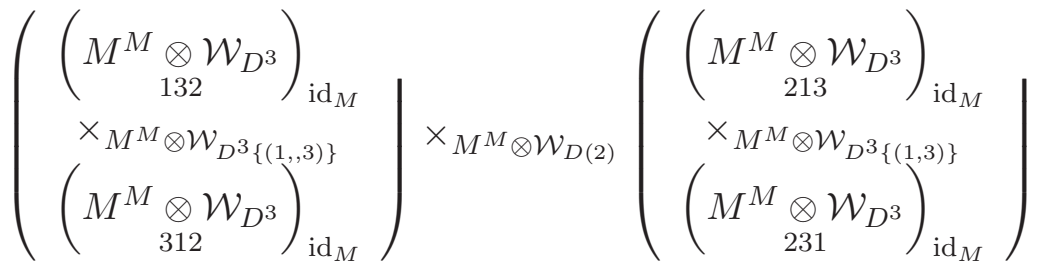

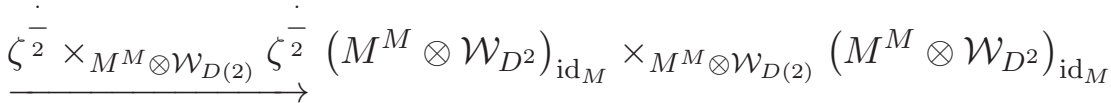

$$
\begin{aligned}
& \stackrel{\dot{\zeta}}{\rightarrow}\left(M^{M} \otimes \mathcal{W}_{D}\right)_{\operatorname{id}_{M}}
\end{aligned}
$$

which is equivalent to

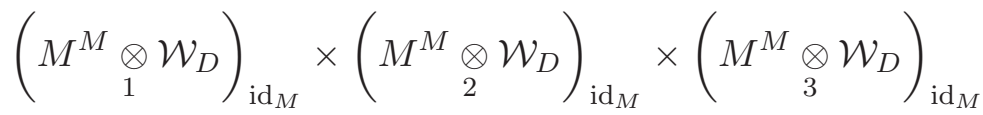

$$
\begin{aligned}
& \left(M_{2}^{M} \underset{2}{\otimes} \mathcal{W}_{D}\right)_{\mathrm{id}_{M}} \times\left(\begin{array}{c}
M^{M} \underset{3}{\otimes} \mathcal{W}_{D} \\
)_{\mathrm{id}_{M}} \\
\times\left(M^{M} \otimes \mathcal{W}_{D}\right. \\
1
\end{array}\right)_{\mathrm{id}_{M}}
\end{aligned}
$$

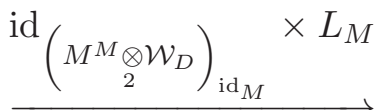

$$
\begin{aligned}
& \left(M_{2}^{M} \otimes \mathcal{W}_{D}\right)_{\mathrm{id}_{M}} \times\left(M^{M} \otimes \mathcal{W}_{D}\right)_{\mathrm{id}_{M}} \stackrel{L_{M}}{\longrightarrow}\left(M^{M} \otimes \mathcal{W}_{D}\right)_{\mathrm{id}_{M}}
\end{aligned}
$$


Proof. The first and the second statements follow from Proposition 37 . The last statement follows from Theorem 36.

Lemma 41. We have the following statements:

1. The composition of morphisms $\chi^{*_{213}}$ and

$$
\left(M^{M} \otimes \mathcal{W}_{D^{3}}\right)_{\operatorname{id}_{M}} \rightarrow\left(M^{M} \otimes \mathcal{W}_{D^{3}\{(1,2)\}}\right)_{\operatorname{id}_{M}}
$$

is equivalent to the composition of morphisms $\chi^{*_{123}}$ and

$$
\left(M^{M} \otimes \mathcal{W}_{D^{3}}\right)_{\mathrm{id}_{M}} \rightarrow\left(M^{M} \otimes \mathcal{W}_{D^{3}\{(1,2)\}}\right)_{\mathrm{id}_{M}}
$$

so that we have

$$
\begin{aligned}
& \left(M_{1}^{M} \otimes \mathcal{W}_{D}\right)_{\mathrm{id}_{M}} \times\left(\begin{array}{c}
M^{M} \underset{2}{\otimes} \mathcal{W}_{D} \\
{ }_{1}
\end{array}\right)_{\mathrm{id}_{M}} \times\left(\begin{array}{c}
M^{M} \otimes \mathcal{W}_{D} \\
3
\end{array}\right)_{\mathrm{id}_{M}} \\
& \stackrel{\left(\chi^{*_{213}}, \chi^{*_{123}}\right)}{\longrightarrow}\left(M^{M} \otimes \mathcal{W}_{D^{3}}\right)_{\mathrm{id}_{M}} \times_{M^{M} \otimes \mathcal{W}_{D^{3}\{(2,3)\}}}\left(M^{M} \otimes \mathcal{W}_{D^{3}}\right)_{\mathrm{id}_{M}} \\
& \stackrel{\zeta^{\overline{3}}}{\rightarrow}\left(M^{M} \otimes \mathcal{W}_{D^{2}}\right)_{\mathrm{id}_{M}}
\end{aligned}
$$

which is equivalent to

$$
\begin{aligned}
& \left(M^{M} \underset{1}{\otimes} \mathcal{W}_{D}\right)_{\mathrm{id}_{M}} \times\left(M^{M} \underset{2}{\otimes} \mathcal{W}_{D}\right)_{\mathrm{id}_{M}} \times\left(M_{3}^{M} \underset{3}{\otimes} \mathcal{W}_{D}\right)_{\mathrm{id}_{M}} \\
& \underline{\left(\left(\chi^{* 31}, \chi^{* 12}\right), \chi^{* 3}\right)} \\
& \left(\left(M^{M} \otimes \mathcal{W}_{D^{2}}\right)_{\mathrm{id}_{M}} \times_{M^{M} \otimes \mathcal{W}_{D(2)}}\left(M^{M} \otimes \mathcal{W}_{D^{2}}\right)_{\mathrm{id}_{M}}\right) \times\left(M^{M} \otimes \mathcal{W}_{D}\right)_{\mathrm{id}_{M}} \\
& \underline{\zeta^{-} \times \operatorname{id}_{M^{M} \otimes \mathcal{W}_{D}}}\left(M^{M} \otimes \mathcal{W}_{D}\right)_{\mathrm{id}_{M}} \times\left(\begin{array}{c}
M^{M} \otimes \mathcal{W}_{D} \\
1
\end{array}\right)_{\operatorname{id}_{M}} \\
& \underset{\operatorname{Ass}_{M}^{1,1}}{\longrightarrow}\left(M^{M} \otimes \mathcal{W}_{D^{2}}\right)_{\mathrm{id}_{M}} \\
& \underset{\operatorname{id}_{M^{M}} \otimes \mathcal{W}_{\left(d_{1}, d_{2}\right) \in D^{2} \mapsto\left(d_{2}, d_{1}\right) \in D^{2}}^{\longrightarrow}}{\longrightarrow}\left(M^{M} \otimes \mathcal{W}_{D^{2}}\right)_{\operatorname{id}_{M}}
\end{aligned}
$$

2. The composition of morphisms $\chi^{* 321}$ and

$$
\left(M^{M} \otimes \mathcal{W}_{D^{3}}\right)_{\mathrm{id}_{M}} \rightarrow\left(M^{M} \otimes \mathcal{W}_{D^{3}\{(2,3)\}}\right)_{\mathrm{id}_{M}}
$$

is equivalent to the composition of morphisms $\chi^{* 312}$ and

$$
\left(M^{M} \otimes \mathcal{W}_{D^{3}}\right)_{\operatorname{id}_{M}} \rightarrow\left(M^{M} \otimes \mathcal{W}_{D^{3}\{(2,3)\}}\right)_{\operatorname{id}_{M}}
$$


so that we have

$$
\begin{aligned}
& \left(\begin{array}{c}
M^{M} \otimes \mathcal{W}_{D} \\
1
\end{array}\right)_{\mathrm{id}_{M}} \times\left(M^{M} \otimes \mathcal{W}_{D}\right)_{\mathrm{id}_{M}} \times\left(M^{M} \otimes \mathcal{W}_{D}\right)_{\mathrm{id}_{M}} \\
& \stackrel{\left(\chi^{* 321}, \chi^{* 312}\right)}{\longrightarrow}\left(M^{M} \otimes \mathcal{W}_{D^{3}}\right)_{\mathrm{id}_{M}} \times{ }_{M^{M} \otimes \mathcal{W}_{D^{3}\{(2,3)\}}}\left(M^{M} \otimes \mathcal{W}_{D^{3}}\right)_{\mathrm{id}_{M}} \\
& \stackrel{\zeta^{\frac{1}{3}}}{\rightarrow}\left(M^{M} \otimes \mathcal{W}_{D^{2}}\right)_{\mathrm{id}_{M}}
\end{aligned}
$$

which is equivalent to

$$
\begin{aligned}
& \left(M^{M} \otimes \mathcal{W}_{D}\right)_{\mathrm{id}_{M}} \times\left(M^{M} \otimes \mathcal{W}_{D}\right)_{\mathrm{id}_{M}} \times\left(M_{3}^{M} \underset{\mathcal{W}_{D}}{ }\right)_{\mathrm{id}_{M}} \\
& \stackrel{\left(\chi^{* 3},\left(\chi^{* 21}, \chi^{* 12}\right)\right)}{\left(M^{M} \otimes \mathcal{W}_{D}\right)_{3 \mathrm{id}_{M}}} \times\left(\left(M^{M} \otimes \mathcal{W}_{D^{2}}\right)_{\mathrm{id}_{M}} \times M^{M} \otimes \mathcal{W}_{D(2)}\left(M^{M} \otimes \mathcal{W}_{D^{2}}\right)_{\mathrm{id}_{M}}\right) \\
& \stackrel{\operatorname{id}_{M^{M} \otimes \mathcal{W}_{D}} \times \zeta^{-}}{\longrightarrow}\left(M^{M} \otimes \mathcal{W}_{D}\right)_{\mathrm{id}_{M}} \times\left(M^{M} \otimes \mathcal{W}_{D}\right) \\
& \stackrel{\operatorname{Ass}_{M}^{1,1}\left(M^{M} \otimes \mathcal{W}_{D^{2}}\right)_{\mathrm{id}_{M}}}{\longrightarrow}
\end{aligned}
$$

3. The composition of (50) and

$$
\left(M^{M} \otimes \mathcal{W}_{D^{2}}\right)_{\mathrm{id}_{M}} \rightarrow\left(M^{M} \otimes \mathcal{W}_{D(2)}\right)_{\mathrm{id}_{M}}
$$

is equivalent to the composition of (52) and

$$
\left(M^{M} \otimes \mathcal{W}_{D^{2}}\right)_{\mathrm{id}_{M}} \rightarrow\left(M^{M} \otimes \mathcal{W}_{D(2)}\right)_{\mathrm{id}_{M}}
$$

in succession, so that we have

$$
\begin{aligned}
& \left(M^{M} \otimes \mathcal{W}_{D}\right)_{\operatorname{id}_{M}} \times\left(\begin{array}{c}
M^{M} \underset{2}{\otimes} \mathcal{W}_{D} \\
)_{\mathrm{id}_{M}} \times\left(M^{M} \underset{3}{\otimes} \mathcal{W}_{D}\right.
\end{array}\right)_{\operatorname{id}_{M}} \\
& \underline{\left(\left(\chi^{* 213}, \chi^{* 123}\right),\left(\chi^{* 321}, \chi^{* 312}\right)\right)}
\end{aligned}
$$

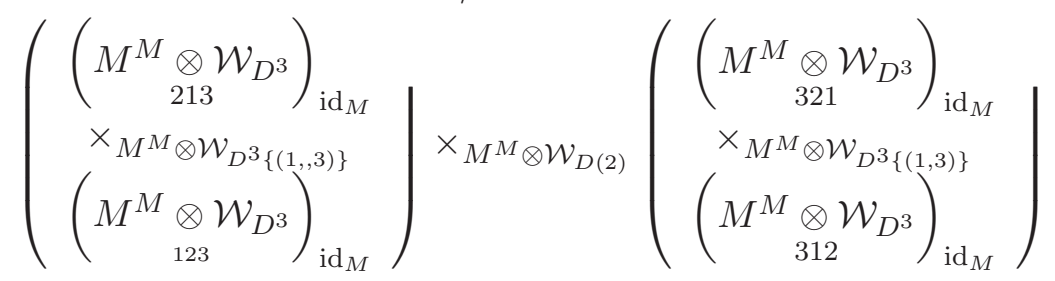




$$
\underset{\stackrel{\zeta^{-}}{\longrightarrow}\left(M^{M} \otimes \mathcal{W}_{D}\right)_{\operatorname{id}_{M}}}{\stackrel{\dot{\overline{3}}}{\longrightarrow}}\left(M^{M} \otimes \mathcal{W}_{D^{2}}\right)_{\mathrm{id}_{M}} \times_{M^{M} \otimes \mathcal{W}_{D(2)}}\left(M^{M} \otimes \mathcal{W}_{D^{2}}\right)_{\mathrm{id}_{M}}
$$

which is equivalent to

$$
\begin{aligned}
& \left(M_{1}^{M} \underset{1}{\otimes} \mathcal{W}_{D}\right)_{\operatorname{id}_{M}} \times\left(M_{2}^{M} \underset{2}{\otimes} \mathcal{W}_{D}\right)_{\operatorname{id}_{M}} \times\left(M_{3}^{M} \underset{3}{\otimes} \mathcal{W}_{D}\right)_{\operatorname{id}_{M}} \\
& \left(M_{3}^{M} \underset{3}{\otimes} \mathcal{W}_{D}\right)_{\mathrm{id}_{M}} \times\left(M_{1}^{M} \underset{1}{\otimes} \mathcal{W}_{D}\right)_{\mathrm{id}_{M}} \times\left(M_{2}^{M} \underset{2}{\otimes \mathcal{W}_{D}}\right)_{\mathrm{id}_{M}} \\
& \stackrel{\mathrm{id}}{\left(M_{3}^{M \otimes} \mathcal{W}_{D}\right)_{\mathrm{id}_{M}}} \times L_{M} \\
& \left(M_{3}^{M} \underset{3}{\otimes} \mathcal{W}_{D}\right)_{\mathrm{id}_{M}} \times\left(M^{M} \otimes \mathcal{W}_{D}\right)_{\mathrm{id}_{M}} \underset{M}{L_{M}}\left(M^{M} \otimes \mathcal{W}_{D}\right)_{\operatorname{id}_{M}}
\end{aligned}
$$

Proof. The first and the second statements follow from Proposition 37. The last statement follows from Theorem 36 .

Theorem 42. (The Conventional Jacobi Identity) We have the following two statements:

1. The three morphisms (41), (47) and (53) sum up only to vanish.

2. The three morphisms (42), (48) and (54) sum up only to vanish.

Proof. We apply the general Jacobi identity to the morphism

$$
\left(M_{1}^{M} \underset{1}{\otimes} \mathcal{W}_{D}\right)_{\operatorname{id}_{M}} \times\left(M_{2}^{M} \underset{2}{\otimes} \mathcal{W}_{D}\right)_{\operatorname{id}_{M}} \times\left(M_{3}^{M} \underset{3}{\otimes} \mathcal{W}_{D}\right)_{\mathrm{id}_{M}}
$$




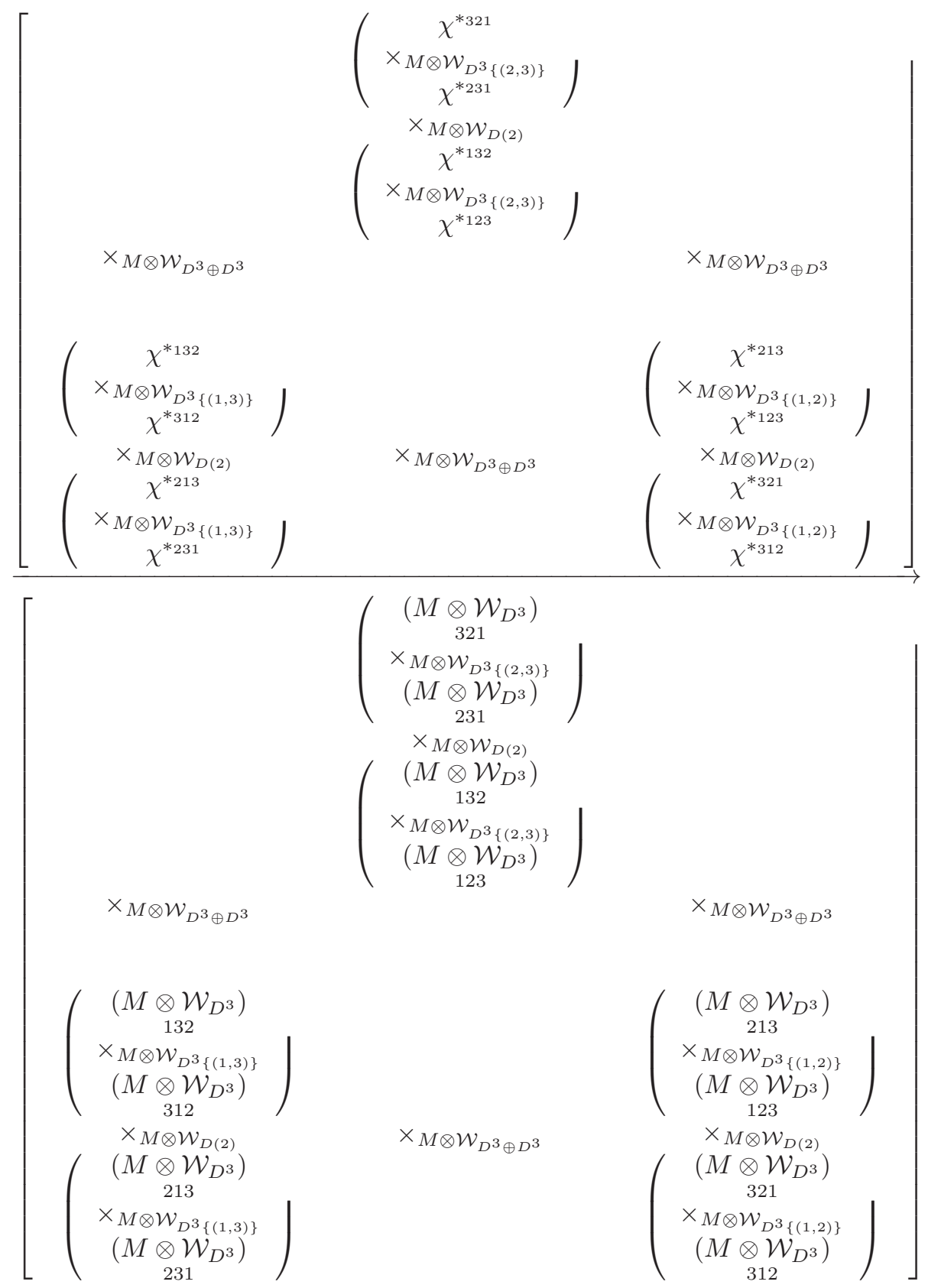

so as to obtain the first statement. The second statement follows directly from the first by Lemmas 39, 40 and 41 . 


\section{References}

[1] René Lavendhomme, Basic Concepts of Synthetic Differential Geometry, Kluwer Academic Publishers, Dordrecht (1996).

[2] Hirokazu Nishimura, Theory of microcubes, International Journal of Theoretical Physics, 36 (1997), 1099-1131.

[3] Hirokazu Nishimura, General Jacobi identity revisited, International Journal of Theoretical Physics, 38 (1999), 2163-2174.

[4] Hirokazu Nishimura, Takeshi Osoekawa, General Jacobi identity revisited again, International Journal of Theoretical Physics, 46 (2007), 2843-2862.

[5] Hirokazu Nishimura, The Jacobi identity beyond Lie algebras, Far East J. Math. Sci., 35 (2009), 33-48.

[6] Hirokazu Nishimura, Differential geometry of microlinear Frölicher spaces I, International Journal of Pure and Applied Mathematics, 64 (2010), 4383.

[7] Hirokazu Nishimura, Axiomatic Differential Geometry I, ArXiv: 1203.3911.

[8] Hirokazu Nishimura, Axiomatic differential geometry II-1, ArXiv: 1204.5230 .

[9] Hirokazu Nishimura, Axiomatic Differential Geometry II-2, ArXiv: 1207.5121.

[10] André Weil, Théorie des points proches sur les variétés différentiables, Colloques Internationaux du Centre National de la Reserche Scientifique, Strassbourg (1953), 111-117. 